\title{
Domino Reaction of 2,4-Diyn-1-ols with 1,3-Dicarbonyl Compounds: Direct access to Aryl/heteroaryl-Fused Benzofurans and Indoles
}

Chada Raji Reddy, ${ }^{* \dagger, *}$ Mounika Aila, ${ }^{\dagger, \dagger}$ Muppidi Subbarao, ${ }^{\dagger, \dagger}$ Kamalkishor Warudikar, ${ }^{\dagger, \dagger}$ and René Grée ${ }^{\neq}$

\footnotetext{
'Department of Organic Synthesis \& Process Chemistry, CSIR-Indian Institute of Chemical Technology, Hyderabad 500007, India. ${ }^{\star}$ Academy of Scientific and Innovative Research (AcSIR), Ghaziabad 201 002, India.

${ }^{\neq}$Univ Rennes, CNRS (Institut for Chemical Sciences in Rennes), UMR 6226, 35000 Rennes, France
}

\section{Table of Contents}

1. General information and starting material preparation - $\quad$ S1-S2

2. Table S1: Optimization of reaction conditions

3. Experimental procedures and characterization data of compounds $\quad-\quad$ S4-S28

4. Gram scale reaction procedure for $\mathbf{3 m} \quad-\quad-\quad S 28$

$\begin{array}{lll}\text { 5. X-Ray analysis data of } 5 & - & \text { S29-S30 }\end{array}$

$\begin{array}{lll}\text { 6. References } & - & \text { S32 }\end{array}$

7. ${ }^{1} \mathrm{H}$ NMR and ${ }^{13} \mathrm{C}$ NMR spectral copies of compounds - $\quad$ S33- S103

\section{1) General information:}

All the reactions were performed in oven-dried glass apparatus, the air and moisture sensitive reactions were carried out under inert atmosphere (nitrogen) using freshly distilled anhydrous solvents. Commercially available reagents were used as such without further purification. All reactions were monitored by thin-layer chromatography carried out on silica plates using UVlight and anisaldehyde for visualization. Column chromatography was performed on silica gel (100-200 mesh) using hexane and ethyl acetate as eluent. ${ }^{1} \mathrm{H}$ NMR was recorded in $\mathrm{CDCl}_{3}$ on $500 \mathrm{MHz}, 400 \mathrm{MHz}$ and $300 \mathrm{MHz}$ and ${ }^{13} \mathrm{C}$ NMR was recorded on $125 \mathrm{MHz}, 100 \mathrm{MHz}$ and $75 \mathrm{MHz} \delta 7.26$ and $\delta 77$ are corresponding to $\mathrm{CDCl}_{3}$ in ${ }^{1} \mathrm{H}$ NMR and ${ }^{13} \mathrm{C}$ NMR respectively, $\delta 1.56$ is related to moisture present in $\mathrm{CDCl}_{3}$. Chemical shifts were reported in $\delta(\mathrm{ppm})$ relative to TMS as an internal standard and $J$ values were given in $\mathrm{Hz}$ (hertz). Multiplicity is indicated as, s (singlet); d (doublet); t (triplet); m (multiplet); dd (doublet of doublets), etc. FT IR spectra were recorded on Alpha (Bruker) Infrared Spectrophotometer. High resolution 
mass spectra (HRMS) [ESI+] were obtain using either a TOF or a double focusing spectrometer. 1,3 dicarbonyl compounds $\mathbf{2 a}$ to $\mathbf{2 f}$ were purchased from commercial source.

\section{Preparation of Starting Materials:}

All the Propargylic alcohols $(\mathbf{1 a}, \mathbf{1 b}, \mathbf{1 f}, \mathbf{1 h}),{ }^{\mathbf{1 a}} \mathbf{1 d},{ }^{1 \mathrm{~b}} \mathbf{1 i},{ }^{1 \mathrm{c}} \mathbf{I},{ }^{\mathbf{1 d}}$ and (II, III) ${ }^{1 \mathrm{e}}$ were prepared based on literature reports and the spectral data was compared.<smiles>OC(C#CC#Cc1ccccc1)c1ccccc1</smiles>

1a<smiles>OC(C#CC#Cc1ccccc1)c1cccc2ccccc12</smiles>

$1 d$<smiles>OC(C#CC#Cc1ccc(C(F)(F)F)cc1)c1ccccc1</smiles>

$1 \mathrm{~g}$<smiles>C#CC(O)c1ccc(-c2ccccc2)cc1</smiles><smiles>COc1ccc(C(O)C#CC#Cc2ccccc2)cc1</smiles>

$1 b$<smiles>CCn1c2ccccc2c2cc(C(O)C#CC#Cc3ccccc3)ccc21</smiles>

$1 e$<smiles>CC(C)(C)C#CC#CC(O)c1ccccc1</smiles>

1h<smiles>C#CC(O)c1ccccc1</smiles>

II<smiles>BrC#Cc1ccccc1</smiles>

III

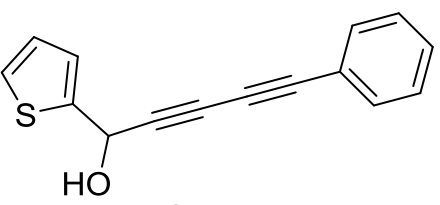

$1 f$<smiles>OC(C#CC#Cc1ccccc1)c1cn([Tl])c2ccccc12</smiles>

$1 i$

IV 


\section{2) Table S1: Optimization of reaction conditions ${ }^{\mathrm{a}}$}<smiles>OC(C#CC#CP)c1ccccc1</smiles>

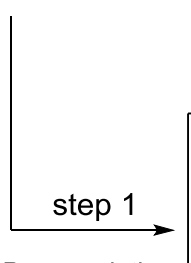

Propargylation<smiles>CCOC(=O)CC(C)=O</smiles><smiles></smiles><smiles>CCOC(=O)c1c(C)n(-c2ccccc2)c2cc(Cc3ccccc3)c3ccccc3c12</smiles><smiles>C[PbH2]c1ccc[nH]1</smiles><smiles>CCOC(=O)c1c(-c2ccccc2)c(CC#Cc2ccccc2)n(-c2ccccc2)c1C</smiles>

B

\begin{tabular}{|c|c|c|c|c|c|c|}
\hline Entry & $\begin{array}{c}\text { step } 1 \\
\text { acid (5 mol\%) }\end{array}$ & Solvent & $\begin{array}{c}\text { step } 2 \\
\text { (amine, temp, time) }\end{array}$ & $\begin{array}{c}\text { step } 3 \\
\text { (Base, temp, time) }\end{array}$ & product & $\begin{array}{l}\text { yield } \\
(\%)^{b}\end{array}$ \\
\hline 1 & $\mathrm{BF}_{3} . \mathrm{Et}_{2} \mathrm{O}, \mathrm{rt}, 30 \mathrm{~min}$ & $\mathrm{CH}_{3} \mathrm{CN}$ & Aniline, $60^{\circ} \mathrm{C}, 7 \mathrm{~h}$ & ---- & B & 40 \\
\hline 2 & PTSA, rt, $30 \mathrm{~min}$ & $\mathrm{CH}_{3} \mathrm{CN}$ & Aniline, $60^{\circ} \mathrm{C}, 7 \mathrm{~h}$ & --- & B & 25 \\
\hline 3 & $\mathrm{Sc}(\mathrm{OTf})_{3}, \mathrm{rt}, 30 \mathrm{~min}$ & $\mathrm{CH}_{3} \mathrm{CN}$ & Aniline, $60^{\circ} \mathrm{C}, 7 \mathrm{~h}$ & --- & B & 30 \\
\hline 4 & $\mathrm{ZnCl}_{2}$, rt, $30 \mathrm{~min}$ & $\mathrm{CH}_{3} \mathrm{CN}$ & Aniline, $60^{\circ} \mathrm{C}, 7 \mathrm{~h}$ & --- & B & 20 \\
\hline 5 & $\ln (\mathrm{OTf})_{3}, \mathrm{rt}, 30 \mathrm{~min}$ & $\mathrm{CH}_{3} \mathrm{CN}$ & Aniline, $60^{\circ} \mathrm{C}, 7 \mathrm{~h}$ & ---- & B & 35 \\
\hline 6 & $\mathrm{BF}_{3} . \mathrm{Et}_{2} \mathrm{O}, 60^{\circ} \mathrm{C}, 1 \mathrm{~h}$ & Toluene & Aniline, $80^{\circ} \mathrm{C}, 8 \mathrm{~h}$ & --- & B & 90 \\
\hline 7 & $\mathrm{BF}_{3} . \mathrm{Et}_{2} \mathrm{O}, 60^{\circ} \mathrm{C}, 1 \mathrm{~h}$ & DCE & Aniline, $80^{\circ} \mathrm{C}, 8 \mathrm{~h}$ & ---- & B & 65 \\
\hline 8 & ---- & ---- & --- & $\mathrm{NaHCO}_{3}, \mathrm{rt}, 4 \mathrm{~h}$ & $4 a$ & 57 \\
\hline 9 & ---- & --- & ---- & $\mathrm{DBU}, \mathrm{rt}, 2 \mathrm{~h}$ & $4 a$ & 92 \\
\hline 10 & ----- & ---- & --- & $\mathrm{K}_{2} \mathrm{CO}_{3}, \mathrm{rt}, 6 \mathrm{~h}$ & $4 a$ & 38 \\
\hline $11^{c}$ & $\mathrm{BF}_{3} \cdot \mathrm{Et}_{2} \mathrm{O}, 60^{\circ} \mathrm{C}, 1 \mathrm{~h}$ & Toluene & Aniline, $80^{\circ} \mathrm{C}, 7 \mathrm{~h}$ & $\mathrm{DBU}, \mathrm{rt}, 2 \mathrm{~h}$ & $4 a$ & $87(8$ \\
\hline
\end{tabular}

a (entries 1 to 7 ): Reaction were performed using $0.5 \mathrm{mmol}$ of $1 \mathrm{a}$ with $0.6 \mathrm{mmol}$ of $\mathbf{2 a}$ in $3 \mathrm{~mL}$ of solvent, 10 mol $\%$ of acid was added at $0{ }^{\circ} \mathrm{C}$. After stirring at $\mathrm{rt}$ for $30 \mathrm{~min}, 0.6 \mathrm{mmol}$ of aniline was added and continued the reaction at $60{ }^{\circ} \mathrm{C}$ for 7 ; (entries 8 to 10): Reaction Int- B with $0.5 \mathrm{mmol}$ base at $\mathrm{rt}$ for $2 \mathrm{~h}$; ${ }^{b}$ Isolated yield; ${ }^{c}$ One-pot reaction; ${ }^{d}$ toluene as solvent. 


\section{3) Experimental procedures and characterization data of compounds:}

\section{A) Experimental Procedures of Diacetylinic Propargylic alcohols:}

\section{1-([1,1'-biphenyl]-4-yl)-5-phenylpenta-2,4-diyn-1-ol (1c):}
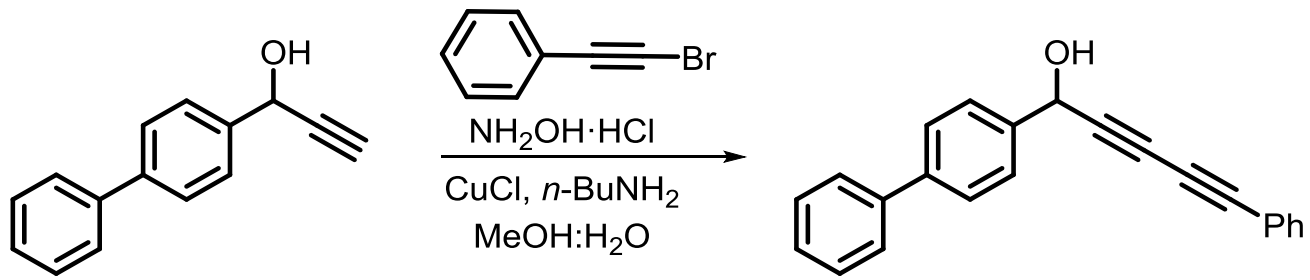

To a stirred solution of 1-([1,1'-biphenyl]-4-yl) prop-2-yn-1-ol I (1 g, $4.8 \mathrm{mmol})$ in Methanol: Water (8:2) (20 mL) were added $\mathrm{CuCl}(47 \mathrm{mg}, 10 \mathrm{~mol} \%), \mathrm{NH}_{2} \mathrm{OH} \cdot \mathrm{HCl}(100 \mathrm{mg}, 30 \mathrm{~mol} \%)$, $n-\mathrm{BuNH}_{2}(1.5 \mathrm{~mL}, 14.4 \mathrm{mmol})$, and (bromo ethynyl) benzene III (860 mg, $\left.4.8 \mathrm{mmol}\right)$ at 0 ${ }^{\circ} \mathrm{C}$, and the solution was warmed to room temperature and stirred for $2 \mathrm{~h}$. The reaction mixture was quenched with $3 \mathrm{~N} \mathrm{HCl}$ and extracted with EtOAc $(2 \times 10 \mathrm{~mL})$. The combined organic layers were washed with brine $(15 \mathrm{~mL})$, dried over $\mathrm{Na}_{2} \mathrm{SO}_{4}$, and concentrated. The residue was purified by flash column chromatography on silica gel (EtOAc in petroleum ether) to afford 1-([1,1'-biphenyl]-4-yl)-5-phenylpenta-2,4-diyn-1-ol 1c, $1.1 \mathrm{~g}, 74 \%$ yield, Pale brown viscous liquid, $R_{f}=0.5$ (petroleum ether: EtOAc $\left.=7: 3\right) ;{ }^{1} \mathrm{H}$ NMR $(400 \mathrm{MHz}$, $\left.\mathrm{CDCl}_{3}\right) \delta 7.77(\mathrm{t}, \mathrm{J}=1.7 \mathrm{~Hz}, 1 \mathrm{H}), 7.65-7.56(\mathrm{~m}, 3 \mathrm{H}), 7.56-7.52(\mathrm{~m}, \mathrm{~J}=7.6,1.3 \mathrm{~Hz}, 1 \mathrm{H})$, $7.52-7.49$ (m, 2H), $7.48-7.43(\mathrm{~m}, 3 \mathrm{H}), 7.40-7.28(\mathrm{~m}, 4 \mathrm{H}), 5.67$ (d, J = 5.1 Hz, 1H), 2.34 $(\mathrm{d}, \mathrm{J}=5.7 \mathrm{~Hz}, 1 \mathrm{H}) ;{ }^{13} \mathrm{C} \mathrm{NMR}\left(125 \mathrm{MHz}, \mathrm{CDCl}_{3}\right) \delta 141.9,140.7,140.2,132.6,129.5,129.3$, $128.8,128.5,127.6,127.3,125.6,121.3,81.5,79.5,73.1,71.4,65.2$; IR (neat): $v \max =1714$, 1128, 764, $704 \mathrm{~cm}^{-1}$; HRMS (ESI): $\mathrm{m} / z$ calcd for $\mathrm{C}_{23} \mathrm{H}_{16} \mathrm{ONa}(\mathrm{M}+\mathrm{Na})^{+}: 331.1099$, found: 331.1108 .

\section{1-(9-ethyl-9H-carbazol-3-yl)-5-phenylpenta-2,4-diyn-1-ol (1e):}
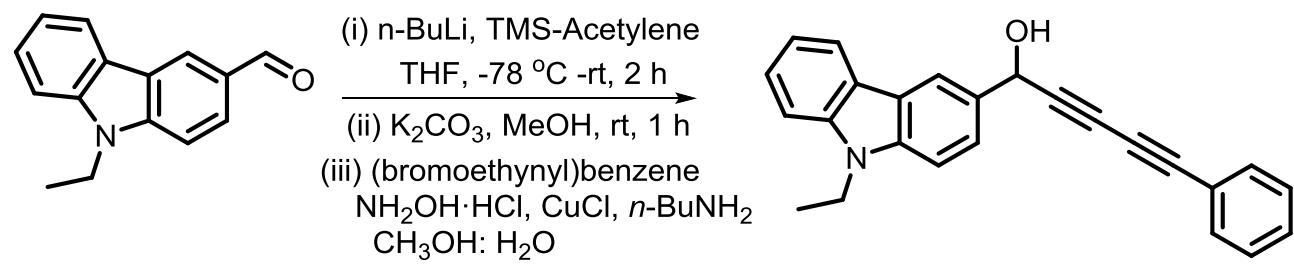

2.5M n-BuLi (5.3 ml, 1.5 equiv) in hexane was added to a solution of TMS acetylene ( $1 \mathrm{~g}$, 1.2 equiv) in dry $\mathrm{THF}(25 \mathrm{~mL})$ at $-78{ }^{\circ} \mathrm{C}$ under nitrogen atmosphere. The mixture was stirred for $30 \mathrm{~min}$ at $-78{ }^{\circ} \mathrm{C}$ before 9-ethyl-9H-carbazole-3-carbaldehyde (2 $\mathrm{g}, 8.9 \mathrm{mmol}$ ) was added. 
The reaction mixture was allowed to stir for $1 \mathrm{~h}$ at room temperature. The aqueous solution was extracted with EtOAc $(3 X 30 \mathrm{~mL})$ after quenching with a saturated aqueous $\mathrm{NH}_{4} \mathrm{Cl}$ solution. The combined organic layers were washed with brine solution $(20 \mathrm{~mL})$. After the organic layer was dried with $\mathrm{Na}_{2} \mathrm{SO}_{4}$, the solvent was removed under reduced pressure to get crude $2^{\circ}$-alcohol $(2.3 \mathrm{~g})$, which was directly subjected for TMS deprotection. To the crude alcohol in $\mathrm{MeOH}(20 \mathrm{~mL}), \mathrm{K}_{2} \mathrm{CO}_{3}(1.5 \mathrm{~g}, 1.5$ equiv) was added and stirred for $2 \mathrm{~h}$ at $\mathrm{rt}$. After that the reaction mixture was filtered on celite pad and washed with ethyl acetate $(2 \times 20 \mathrm{~mL})$. The filtrate was concentrated under reduced pressure, obtained concentrated to get crude product of 1-(9-ethyl-9H-carbazol-3-yl) prop-2-yn-1-ol (1.3 g).

To a stirred solution of 1-(9-ethyl-9H-carbazol-3-yl) prop-2-yn-1-ol (1.3 g, $5.2 \mathrm{mmol})$ in $\mathrm{CH}_{3} \mathrm{OH}: \mathrm{H}_{2} \mathrm{O}$ (8:2) were added $\mathrm{CuCl}(70 \mathrm{mg}, 10 \mathrm{~mol} \%), \mathrm{NH}_{2} \mathrm{OH} \cdot \mathrm{HCl}(108 \mathrm{mg}, 30 \mathrm{~mol} \%), n-$ $\mathrm{BuNH}_{2}(1.54 \mathrm{~mL}, 15.6 \mathrm{mmol})$, and 1-bromooct-1-yne III (939 mg, $\left.5.2 \mathrm{mmol}\right)$ at $0{ }^{\circ} \mathrm{C}$, and the solution was warmed to room temperature and stirred for $2 \mathrm{~h}$. The reaction mixture was quenched with $3 \mathrm{~N} \mathrm{HCl}$ and extracted with EtOAc $(2 \times 30 \mathrm{~mL})$. The combined organic layers were washed with brine $(25 \mathrm{~mL})$, dried over $\mathrm{Na}_{2} \mathrm{SO}_{4}$, and concentrated. The residue was purified by flash column chromatography on silica gel (EtOAc in petroleum ether) to afford 1-(9-ethyl-9H-carbazol-3-yl)-5-phenylpenta-2,4-diyn-1-ol 1e, $1.5 \mathrm{~g}, 48 \%$ yield, brown viscous liquid, $R_{f}=0.4$ (petroleum ether $:$ EtOAc $\left.=8: 2\right) ;{ }^{1} \mathrm{H} \mathrm{NMR}\left(400 \mathrm{MHz}, \mathrm{CDCl}_{3}\right) \delta 8.27$ $(\mathrm{d}, J=1.7 \mathrm{~Hz}, 1 \mathrm{H}), 8.13(\mathrm{~d}, J=7.8 \mathrm{~Hz}, 1 \mathrm{H}), 7.66(\mathrm{dd}, J=8.5,1.8 \mathrm{~Hz}, 1 \mathrm{H}), 7.53-7.45$ (m, $3 \mathrm{H}), 7.44-7.29(\mathrm{~m}, 5 \mathrm{H}), 7.27-7.22(\mathrm{~m}, 1 \mathrm{H}), 5.79(\mathrm{~s}, 1 \mathrm{H}), 4.37$ (q, J = 7.2 Hz, 2H), 1.43 (t, $J=7.2 \mathrm{~Hz}, 3 \mathrm{H}) ;{ }^{13} \mathrm{C}$ NMR $\left(100 \mathrm{MHz}, \mathrm{CDCl}_{3}\right) \delta 140.4,140.1,132.6,130.5,129.4,128.5$, 126.0, 124.7, 123.1, 122.9, 121.5, 120.7, 119.1, 119.1, 108.8, 108.7, 82.5, 79.4, 73.5, 71.2, 65.8, 37.7, 13.8; IR (neat): $v \max =1699,1514,1412,1172,762 \mathrm{~cm}^{-1} ; \mathrm{HRMS}(\mathrm{ESI}): \mathrm{m} / \mathrm{z}$ calcd for $\mathrm{C}_{25} \mathrm{H}_{18} \mathrm{~N}\left(\mathrm{M}-\mathrm{H}_{2} \mathrm{O}\right)^{+}: 332.1439$, found: 332.1440 .

\section{1-phenyl-5-(4-(trifluoromethyl) phenyl) penta-2,4-diyn-1-ol (1g):}

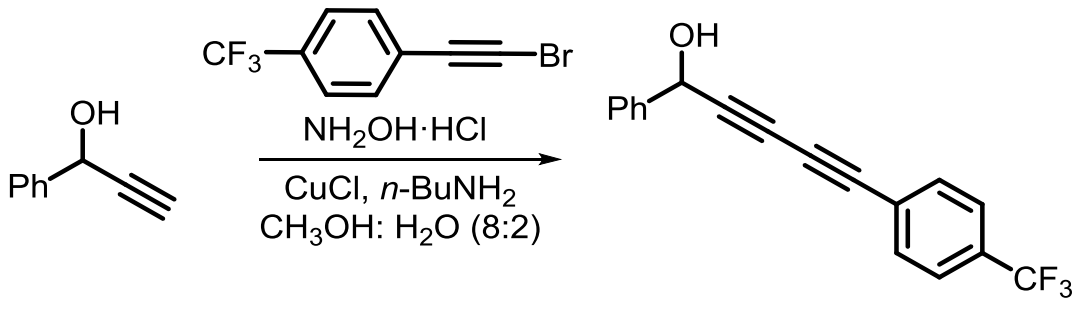

To a stirred solution of 1-phenylprop-2-yn-1-ol II (1 g, $7.57 \mathrm{mmol})$ in Methanol: Water (8:2) $(20 \mathrm{~mL})$ were added $\mathrm{CuCl}(101 \mathrm{mg}, 10 \mathrm{~mol} \%), \mathrm{NH}_{2} \mathrm{OH} \cdot \mathrm{HCl}(157 \mathrm{mg}, 30 \mathrm{~mol} \%), n-$ 
$\mathrm{BuNH}_{2}(2.3 \mathrm{~mL}, 22.71 \mathrm{mmol})$, and 1-(bromoethynyl)-4-(trifluoromethyl) benzene IV (1.87 g, $7.57 \mathrm{mmol}$ ) at $0{ }^{\circ} \mathrm{C}$, and the solution was warmed to room temperature and stirred for $2 \mathrm{~h}$. The reaction mixture was quenched with $3 \mathrm{~N} \mathrm{HCl}$ and extracted with EtOAc $(2 \times 10 \mathrm{~mL})$. The combined organic layers were washed with brine $(15 \mathrm{~mL})$, dried over $\mathrm{Na}_{2} \mathrm{SO}_{4}$, and concentrated. The residue was purified by flash column chromatography on silica gel (EtOAc in petroleum ether) to afford propargylic alcohol 2l, $1.1 \mathrm{~g}, 74 \%$ yield, Pale brown viscous liquid, $R_{f}=0.4$ (petroleum ether: EtOAc $\left.=8: 2\right) ;{ }^{1} \mathrm{H}$ NMR $\left(400 \mathrm{MHz}, \mathrm{CDCl}_{3}\right) \delta 7.61-7.53$ $(\mathrm{m}, 6 \mathrm{H}), 7.45-7.36(\mathrm{~m}, 3 \mathrm{H}), 5.61(\mathrm{~s}, 1 \mathrm{H}) ;{ }^{13} \mathrm{C} \mathrm{NMR}\left(101 \mathrm{MHz}, \mathrm{CDCl}_{3}\right) \delta 139.4,132.8$, 128.8 (2C), 126.7, 126.6, 125.4, 125.4, 123.7 (d, $J=272.3 \mathrm{~Hz}), 82.9,77.7,75.4$, 70.8, 65.2; IR (neat): vmax $=3027,2359,1612,1485,1447,748,700 \mathrm{~cm}^{-1} ;$ HRMS $(E S I): \mathrm{m} / \mathrm{z}$ calcd for $\mathrm{C}_{18} \mathrm{H}_{10} \mathrm{~F}_{3}\left(\mathrm{M}-\mathrm{H}_{2} \mathrm{O}\right)^{+}:$283.0735, found: 283.0733.

\section{Ethyl 2-acetyl-3,7-diphenylhepta-4,6-diynoate (A):}
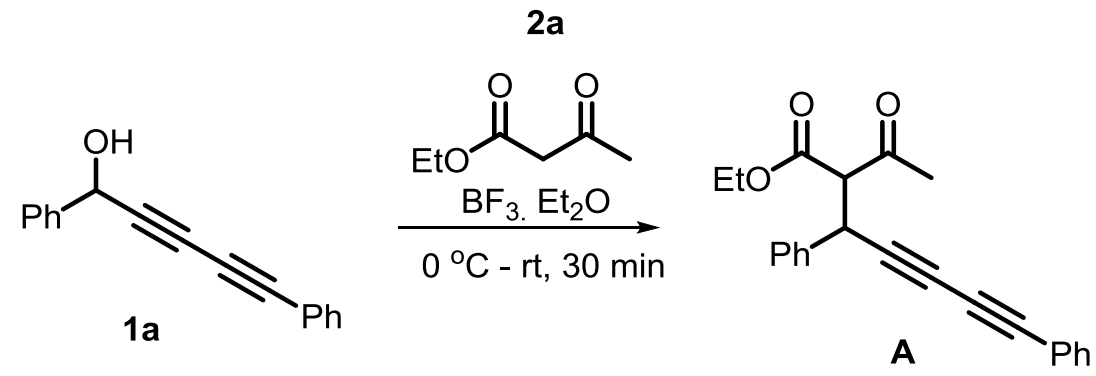

To a stirred solution of 1,5-diphenylpenta-2,4-diyn-1-ol $\mathbf{1 a}(116 \mathrm{mg}, 0.5 \mathrm{mmol})$ and ethyl 3oxobutanoate $2 \mathbf{a}(45 \mathrm{mg}, 0.35 \mathrm{mmol})$ in $3 \mathrm{~mL}$ of acetonitrile in a reaction vial was added $\mathrm{BF}_{3} \cdot \mathrm{Et}_{2} \mathrm{O}(7 \mathrm{mg}, 10 \mathrm{~mol} \%)$ at $0{ }^{\circ} \mathrm{C}$ and moved to room temperature and stirred for $30 \mathrm{~min}$. After completion of the reaction (monitored by TLC) the mixture was concentrated under reduced pressure. The crude product was purified by column chromatography on silica gel by using EtOAc: hexane as an eluent to afford the diastereomer mixture (50:50) of Ethyl 2acetyl-3,7-diphenylhepta-4,6-diynoate A, $163 \mathrm{mg}, 95 \%$ yield, pale yellow liquid; $R_{f}=0.5$ (hexane: EtOAc = 9:1); ${ }^{1} \mathrm{H}$ NMR $\left(400 \mathrm{MHz}, \mathrm{CDCl}_{3}\right) \delta 7.48-7.42(\mathrm{~m}, 2 \mathrm{H}), 7.40-7.25(\mathrm{~m}$, $8 \mathrm{H}), 4.61-4.51(\mathrm{~m}, J=13.5,10.5 \mathrm{~Hz}, 2 \mathrm{H}), 4.28(\mathrm{q}, J=7.1 \mathrm{~Hz}, 2 \mathrm{H}), 4.08-3.89(\mathrm{~m}, 4 \mathrm{H})$, 2.39 (s, 3H), 1.99 (s, 3H), $1.33(\mathrm{t}, J=7.1 \mathrm{~Hz}, 3 \mathrm{H}), 1.02(\mathrm{t}, J=7.1 \mathrm{~Hz}, 3 \mathrm{H}) ;{ }^{13} \mathrm{C} \mathrm{NMR}(100$ $\left.\mathrm{MHz}, \mathrm{CDCl}_{3}\right) \delta 200.2,199.9,166.9,166.4,137.2,137.1,132.6,129.3,129.2,129.0,128.9$, $128.5,128.4,128.4,128.0,121.7,121.6,82.2,81.9,73.9,73.8,69.0,68.7,66.2,65.8,62.2$, 61.8, 37.9, 37.9, 30.8, 30.0, 14.2, 13.9; IR (neat): $v \max =1737,1363,1258,1176,761 \mathrm{~cm}^{-1}$; HRMS (ESI): $m / z$ calcd for $\mathrm{C}_{23} \mathrm{H}_{21} \mathrm{O}_{3}(\mathrm{M}+\mathrm{H})^{+}: 345.1491$, found: 345.1488 .

\section{B) General Procedure: Synthesis of Annulated-Benzofurans}




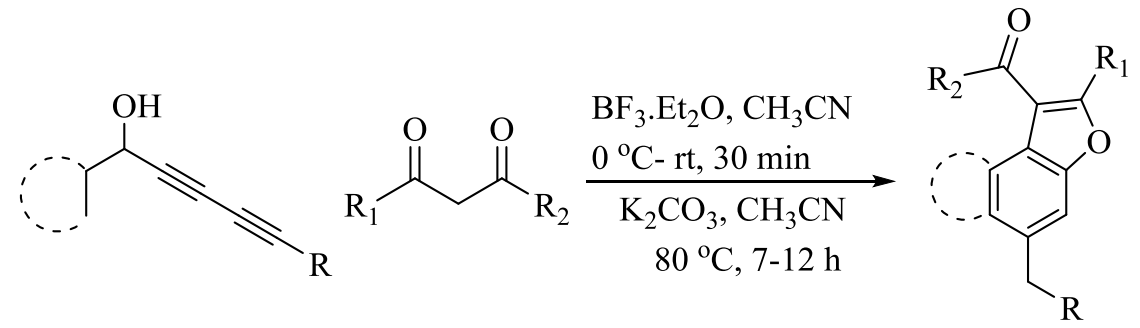

To a stirred solution of diacetylinic propargylic alcohol $1(0.5 \mathrm{mmol})$ and 1,3-dicarbonyl compounds $2(0.35 \mathrm{mmol})$ in $3 \mathrm{~mL}$ of acetonitrile in a reaction vial was added $\mathrm{BF}_{3} \cdot \mathrm{Et}_{2} \mathrm{O}(10$ mol\%) at $0{ }^{\circ} \mathrm{C}$ and moved to room temperature and stirred for $30 \mathrm{~min}$. Then, $\mathrm{K}_{2} \mathrm{CO}_{3}(1.25$ mmol) was added and stirred at $80{ }^{\circ} \mathrm{C}$ (oil bath temperature) for 7-12 h. After completion of the reaction (monitored by TLC) the mixture was concentrated under reduced pressure. The crude product was purified by column chromatography on silica gel by using EtOAc: hexane as an eluent to afford the corresponding napthofuran $\mathbf{3}$.

Ethyl 5-benzyl-2-methylnaphtho[2,1-b] furan-1-carboxylate (3a):

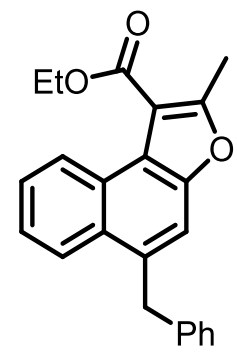

Following the general procedure, to the solution of 1,5-diphenylpenta-2,4-diyn-1-ol 1a (116 mg, $0.5 \mathrm{mmol})$ and ethyl 3-oxobutanoate $\mathbf{2 a}(45 \mathrm{mg}, 0.35 \mathrm{mmol})$ in $3 \mathrm{~mL}$ of acetonitrile in a reaction vial was added $\mathrm{BF}_{3} \cdot \mathrm{Et}_{2} \mathrm{O}(7 \mathrm{mg}, 10 \mathrm{~mol} \%)$ at $0{ }^{\circ} \mathrm{C}$ and moved to room temperature and stirred for $30 \mathrm{~min}$. Then, $\mathrm{K}_{2} \mathrm{CO}_{3}\left(172 \mathrm{mg}, 1.25 \mathrm{mmol}\right.$ ) was added and stirred at $80{ }^{\circ} \mathrm{C}$ (oil bath temperature) for $7 \mathrm{~h}$. After completion of the reaction (monitored by TLC), the reaction mixture was concentrated under reduced pressure. The crude product was purified by column chromatography on silica gel (EtOAc-hexane) to afford the Ethyl 5-benzyl-2methylnaphtho[2,1-b]furan-1-carboxylate 3a, $158 \mathrm{mg}, 92 \%$ yield, off-white solid; $R_{f}=0.7$ (hexane: EtOAc = 9:1); mp: $119-121{ }^{\circ} \mathrm{C} ;{ }^{1} \mathrm{H}$ NMR $\left(400 \mathrm{MHz}, \mathrm{CDCl}_{3}\right) \delta 9.23(\mathrm{~d}, J=8.5 \mathrm{~Hz}$, 1H), $8.07(\mathrm{~d}, J=8.3 \mathrm{~Hz}, 1 \mathrm{H}), 7.59-7.55(\mathrm{~m}, 1 \mathrm{H}), 7.49-7.44(\mathrm{~m}, 1 \mathrm{H}), 7.37(\mathrm{~s}, 1 \mathrm{H}), 7.31-$ $7.26(\mathrm{~m}, 2 \mathrm{H}), 7.23-7.18(\mathrm{~m}, 3 \mathrm{H}), 4.52-4.47(\mathrm{~m}, 4 \mathrm{H}), 2.76(\mathrm{~s}, 3 \mathrm{H}), 1.48(\mathrm{t}, J=7.1 \mathrm{~Hz}, 3 \mathrm{H})$; ${ }^{13} \mathrm{C}$ NMR $\left(125 \mathrm{MHz}, \mathrm{CDCl}_{3}\right) \delta 165.2,161.0,151.3,140.2,135.1,129.98,128.9,128.6$, $128.4,126.7,126.3,126.0,124.9,124.8,119.4,113.1,111.8,60.9,39.5,15.3,14.4$; IR 
$(\mathrm{KBr}): v \max =1698,1179,1123,1073,759 \mathrm{~cm}^{-1} ;$ HRMS $(\mathrm{ESI}): \mathrm{m} / z$ calcd for $\mathrm{C}_{23} \mathrm{H}_{21} \mathrm{O}_{3}$ $(\mathrm{M}+\mathrm{H})^{+}:$345.1491, found: 345.1488 .

Ethyl 5-benzyl-7-methoxy-2-methylnaphtho[2,1-b] furan-1-carboxylate (3b):<smiles>CCOC(=O)c1c(C)oc2cc(Cc3ccccc3)c3cc(OC)ccc3c12</smiles>

Following the general procedure, to the solution of 1-(4-methoxyphenyl)-5-phenylpenta-2,4-diyn1-ol $1 \mathbf{b}(131 \mathrm{mg}, 0.5 \mathrm{mmol})$ and ethyl 3-oxobutanoate $\mathbf{2 a}(45 \mathrm{mg}, 0.35 \mathrm{mmol})$ in $3 \mathrm{~mL}$ of acetonitrile in a reaction vial was added $\mathrm{BF}_{3} \cdot \mathrm{Et}_{2} \mathrm{O}(7 \mathrm{mg}, 10 \mathrm{~mol} \%)$ at $0{ }^{\circ} \mathrm{C}$ and moved to room temperature and stirred for $30 \mathrm{~min}$. Then, $\mathrm{K}_{2} \mathrm{CO}_{3}(172 \mathrm{mg}, 1.25 \mathrm{mmol})$ was added and stirred at $80{ }^{\circ} \mathrm{C}$ (oil bath temperature) for $8 \mathrm{~h}$. After completion of the reaction (monitored by TLC), the reaction mixture was concentrated under reduced pressure. The crude product was purified by column chromatography on silica gel (EtOAc-hexane) to afford the Ethyl 5benzyl-7-methoxy-2-methylnaphtho[2,1-b] furan-1-carboxylate 3b, $170 \mathrm{mg}$, 91\%, Off-white solid; $R_{f}=0.5$ (hexane: EtOAc $=9: 1$ ); mp 128-131 ${ }^{\circ} \mathrm{C} ;{ }^{1} \mathrm{H} \mathrm{NMR}\left(400 \mathrm{MHz}, \mathrm{CDCl}_{3}\right) \delta 9.19$ $(\mathrm{d}, J=9.3 \mathrm{~Hz}, 1 \mathrm{H}), 7.37(\mathrm{~s}, 1 \mathrm{H}), 7.35(\mathrm{~d}, J=2.6 \mathrm{~Hz}, 1 \mathrm{H}), 7.30-7.26(\mathrm{~m}, 2 \mathrm{H}), 7.25-7.18$ $(\mathrm{m}, 4 \mathrm{H}), 4.48(\mathrm{q}, J=7.1 \mathrm{~Hz}, 2 \mathrm{H}), 4.44(\mathrm{~s}, 2 \mathrm{H}), 3.81(\mathrm{~s}, 3 \mathrm{H}), 2.75(\mathrm{~s}, 3 \mathrm{H}), 1.47$ (t, $J=7.1 \mathrm{~Hz}$, $3 \mathrm{H}) ;{ }^{13} \mathrm{C}$ NMR $\left(125 \mathrm{MHz}, \mathrm{CDCl}_{3}\right) \delta 165.2,161.2,156.5,150.3,140.1,133.8,131.3,128.8$, 128.6, 128.3, 126.3, 123.1, 119.6, 117.1, 113.4, 111.5, 104.9, 60.8, 55.2, 39.9, 15.4, 14.4; IR $(\mathrm{KBr}): v \max =1711,1234,1178,1115,839 \mathrm{~cm}^{-1} ; \mathrm{HRMS}(\mathrm{ESI}): \mathrm{m} / z$ calcd for $\mathrm{C}_{24} \mathrm{H}_{23} \mathrm{O}_{4}$ $(\mathrm{M}+\mathrm{H})^{+}:$375.1596, found: 375.1603 .

\section{Ethyl 5-benzyl-2-methyl-7-phenylnaphtho[2,1-b]furan-1-carboxylate (3c):}

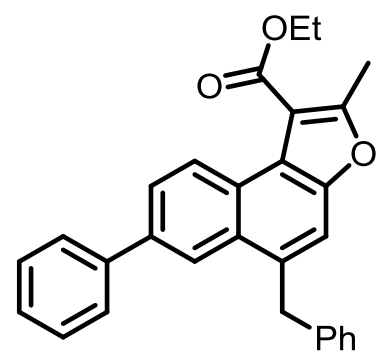

Following the general procedure, to the solution 1-(1,1'-biphenyl]-4-yl)-5-phenylpenta-2,4-diyn-1ol 1c (154 mg, $0.5 \mathrm{mmol}$ ) and ethyl 3-oxobutanoate 2a (45 mg, $0.35 \mathrm{mmol})$ in $3 \mathrm{~mL}$ of acetonitrile in a reaction vial was added $\mathrm{BF}_{3} \cdot \mathrm{Et}_{2} \mathrm{O}(7 \mathrm{mg}, 10 \mathrm{~mol} \%)$ at $0{ }^{\circ} \mathrm{C}$ and moved to 
room temperature and stirred for $30 \mathrm{~min}$. Then, $\mathrm{K}_{2} \mathrm{CO}_{3}(172 \mathrm{mg}, 1.25 \mathrm{mmol})$ was added and stirred at $80{ }^{\circ} \mathrm{C}$ (oil bath temperature) for $9 \mathrm{~h}$. After completion of the reaction (monitored by TLC), the reaction mixture was concentrated under reduced pressure. The crude product was purified by column chromatography on silica gel (EtOAc-hexane) to afford the Ethyl 5benzyl-2-methyl-7-phenylnaphtho[2,1-b]furan-1-carboxylate 3c, $175 \mathrm{mg}$, 83\% yield, Brown solid; $R_{f}=0.6$ (hexane: EtOAc $\left.=9: 1\right) ; \operatorname{mp~} 145-147{ }^{\circ} \mathrm{C} ;{ }^{1} \mathrm{H}$ NMR $\left(400 \mathrm{MHz}, \mathrm{CDCl}_{3}\right) \delta 9.53$ $(\mathrm{d}, \mathrm{J}=1.9 \mathrm{~Hz}, 1 \mathrm{H}), 8.13(\mathrm{~d}, \mathrm{~J}=8.8 \mathrm{~Hz}, 1 \mathrm{H}), 7.80(\mathrm{~d}, \mathrm{~J}=7.3 \mathrm{~Hz}, 2 \mathrm{H}), 7.74(\mathrm{dd}, \mathrm{J}=8.8,1.9$ $\mathrm{Hz}, 1 \mathrm{H}), 7.53-7.46(\mathrm{~m}, 2 \mathrm{H}), 7.40-7.34(\mathrm{~m}, 2 \mathrm{H}), 7.33-7.26(\mathrm{~m}, 2 \mathrm{H}), 7.25-7.17(\mathrm{~m}, 3 \mathrm{H})$, $4.55-4.45(\mathrm{~m}, 4 \mathrm{H}), 2.77(\mathrm{~s}, 3 \mathrm{H}), 1.43(\mathrm{t}, \mathrm{J}=7.1 \mathrm{~Hz}, 3 \mathrm{H}) ;{ }^{13} \mathrm{C}$ NMR $\left(100 \mathrm{MHz}, \mathrm{CDCl}_{3}\right) \delta$ 165.1, 161.2, 151.6, 141.3, 140.1, 138.4, 134.9, 129.1, 128.9 (2C), 128.7, 128.6, 127.5, 127.3, 126.3, 125.5, 124.8, 124.1, 119.6, 113.1, 111.9, 60.9, 39.5, 15.3, 14.4; IR (KBr): $v_{\max }=1714$, 1392, 1128, 764, $704 \mathrm{~cm}^{-1}$; HRMS (ESI): $m / z$ calcd for $\mathrm{C}_{29} \mathrm{H}_{25} \mathrm{O}_{3}(\mathrm{M}+\mathrm{H})^{+}: 421.1804$, found: 421.1801 .

\section{Ethyl 5-benzyl-2-methylbenzo[7,8]naphtho[2,1-b]furan-1-carboxylate(3d):}

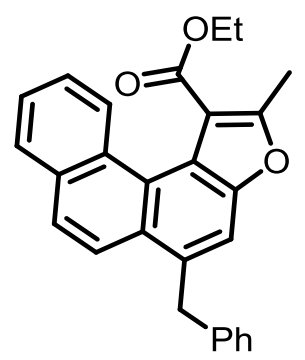

Following the general procedure, to the solution of 1-(naphthalen-1-yl)-5-phenylpenta-2,4-diyn-1ol 1d (141 mg, $0.5 \mathrm{mmol}$ ) and ethyl 3-oxobutanoate 2a (45 mg, $0.35 \mathrm{mmol}$ ) in $3 \mathrm{~mL}$ of acetonitrile in a reaction vial was added $\mathrm{BF}_{3} \cdot \mathrm{Et}_{2} \mathrm{O}(7 \mathrm{mg}, 10 \mathrm{~mol} \%)$ at $0{ }^{\circ} \mathrm{C}$ and moved to room temperature and stirred for $30 \mathrm{~min}$. Then, $\mathrm{K}_{2} \mathrm{CO}_{3}(172 \mathrm{mg}, 1.25 \mathrm{mmol})$ was added and stirred at $80{ }^{\circ} \mathrm{C}$ (oil bath temperature) for $8 \mathrm{~h}$. After completion of the reaction (monitored by TLC), the reaction mixture was concentrated under reduced pressure. The crude product was purified by column chromatography on silica gel (EtOAc-hexane) to afford the Ethyl 5benzyl-2-methylbenzo[7,8]naphtho[2,1-b]furan-1-carboxylate 3d, $170 \mathrm{mg}, 86 \%$ yield, Offwhite solid; $R_{f}=0.4$ (hexane: EtOAc $\left.=9: 1\right) ; \operatorname{mp~218-220~}{ }^{\circ} \mathrm{C} ;{ }^{1} \mathrm{H}$ NMR $\left(300 \mathrm{MHz}, \mathrm{CDCl}_{3}\right) \delta$ $8.21(\mathrm{~d}, J=8.1 \mathrm{~Hz}, 1 \mathrm{H}), 7.99(\mathrm{~d}, J=9.1 \mathrm{~Hz}, 1 \mathrm{H}), 7.88(\mathrm{~d}, J=7.5 \mathrm{~Hz}, 1 \mathrm{H}), 7.72(\mathrm{~d}, J=9.1$ $\mathrm{Hz}, 1 \mathrm{H}), 7.59-7.46(\mathrm{~m}, 3 \mathrm{H}), 7.32-7.26(\mathrm{~m}, 2 \mathrm{H}), 7.22$ (d, J = 7.2 Hz, 3H), $4.56(\mathrm{~s}, 2 \mathrm{H})$, $4.12(\mathrm{t}, J=7.1 \mathrm{~Hz}, 2 \mathrm{H}), 2.77(\mathrm{~s}, 3 \mathrm{H}), 0.76(\mathrm{t}, J=7.1 \mathrm{~Hz}, 3 \mathrm{H}) ;{ }^{13} \mathrm{C} \mathrm{NMR}\left(100 \mathrm{MHz}, \mathrm{CDCl}_{3}\right) \delta$ $165.9,160.1,153.2,140.3,134.8,132.1,130.0,128.8$ (2C), 128.6, 128.1, 127.9, 127.3, 126.3, $125.8,125.7,124.2,123.0,118.6,113.2,112.9,60.9,39.6,13.7,13.3$; IR $(\mathrm{KBr}): v \max =$ 
1715, 1450, 1258, 1102, $760 \mathrm{~cm}^{-1}$; HRMS (ESI): $\mathrm{m} / \mathrm{z}$ calcd for $\mathrm{C}_{27} \mathrm{H}_{22} \mathrm{O}_{3} \mathrm{Na}(\mathrm{M}+\mathrm{Na})^{+}$: 417.1467, found: 417.1478 .

\section{Ethyl 5-benzyl-7-ethyl-2-methyl-7H-benzofuro[5,4-b]carbazole-1-carboxylate(3e):}

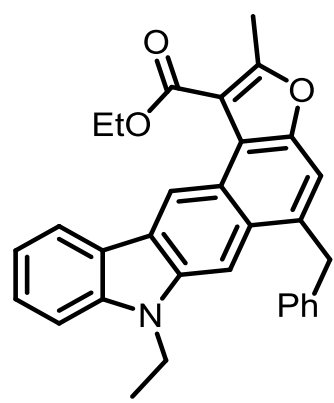

Following the general procedure, to the solution 1-(9-ethyl-9H-carbazol-3-yl)-5-phenylpenta-2,4diyn-1-ol 1e (174 mg, $0.5 \mathrm{mmol})$ and ethyl 3-oxobutanoate $\mathbf{2 a}(45 \mathrm{mg}, 0.35 \mathrm{mmol})$ in $3 \mathrm{~mL}$ of acetonitrile in a reaction vial was added $\mathrm{BF}_{3} \cdot \mathrm{Et}_{2} \mathrm{O}(7 \mathrm{mg}, 10 \mathrm{~mol} \%)$ at $0{ }^{\circ} \mathrm{C}$ and moved to room temperature and stirred for $30 \mathrm{~min}$. Then, $\mathrm{K}_{2} \mathrm{CO}_{3}(172 \mathrm{mg}, 1.25 \mathrm{mmol})$ was added and stirred at $80{ }^{\circ} \mathrm{C}$ (oil bath temperature) for $7 \mathrm{~h}$. After completion of the reaction (monitored by TLC), the reaction mixture was concentrated under reduced pressure. The crude product was purified by column chromatography on silica gel (EtOAc-hexane) to afford the Ethyl 5benzyl-7-ethyl-2-methyl-7H-benzofuro[5,4-b]carbazole-1-carboxylate $\mathbf{3 e}, 191 \mathrm{mg}, \quad 83 \%$ yield, off-white solid; $R_{f}=0.7$ (hexane: EtOAc $=9: 1$ ); mp $184-186{ }^{\circ} \mathrm{C} ;{ }^{1} \mathrm{H}$ NMR $(400 \mathrm{MHz}$, $\left.\mathrm{CDCl}_{3}\right) \delta 9.22(\mathrm{~d}, J=9.2 \mathrm{~Hz}, 1 \mathrm{H}), 8.27(\mathrm{~d}, J=8.2 \mathrm{~Hz}, 1 \mathrm{H}), 7.75(\mathrm{~d}, J=9.2 \mathrm{~Hz}, 1 \mathrm{H}), 7.55(\mathrm{~d}$, $J=8.2 \mathrm{~Hz}, 1 \mathrm{H}), 7.47-7.39(\mathrm{~m}, 2 \mathrm{H}), 7.20(\mathrm{dd}, J=15.2,7.8 \mathrm{~Hz}, 3 \mathrm{H}), 7.13(\mathrm{t}, J=7.2 \mathrm{~Hz}, 1 \mathrm{H})$, $7.03(\mathrm{~d}, J=7.4 \mathrm{~Hz}, 2 \mathrm{H}), 4.99$ (s, 2H), $4.60-4.54$ (m, 2H), $4.54-4.48$ (m, 2H), 2.74 (s, 3H), $1.52(\mathrm{t}, J=7.7 \mathrm{~Hz}, 3 \mathrm{H}), 1.48(\mathrm{t}, J=7.2 \mathrm{~Hz}, 3 \mathrm{H}) ;{ }^{13} \mathrm{C} \mathrm{NMR}\left(100 \mathrm{MHz}, \mathrm{CDCl}_{3}\right) \delta 165.5,161.3$, $150.4,143.0,138.7,138.4,134.6,129.3,128.3$, 127.6, 125.8, 125.5, 124.8, 124.3, 123.7, 123.4, 120.2, 118.4, 115.5, 114.1, 111.6, 109.7, 108.7, 60.8, 42.0, 37.8, 15.4, 14.4, 14.2; IR $(\mathrm{KBr}): v \max =1695,1395,1172,1098,755 \mathrm{~cm}^{-1} ; \mathrm{HRMS}(\mathrm{ESI}): \mathrm{m} / z$ calcd for $\mathrm{C}_{31} \mathrm{H}_{28} \mathrm{NO}_{3}$ $(\mathrm{M}+\mathrm{H})^{+}:$462.2069, found: 462.2063 .

Ethyl 4-benzyl-7-methylthieno[2,3-e] benzofuran-8-carboxylate (3f):

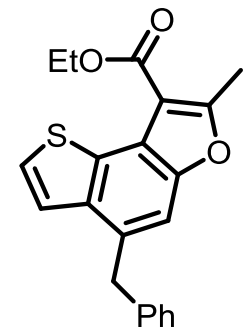

Following the general procedure, to the solution 5-phenyl-1-(thiophen-3-yl)penta-2,4-diyn-1-ol 1f (119 mg, $0.5 \mathrm{mmol}$ ) and ethyl 3-oxobutanoate $2 \mathbf{a}(45 \mathrm{mg}, 0.35 \mathrm{mmol})$ in $3 \mathrm{~mL}$ of acetonitrile in a 
reaction vial was added $\mathrm{BF}_{3} \cdot \mathrm{Et}_{2} \mathrm{O}(7 \mathrm{mg}, 10 \mathrm{~mol} \%)$ at $0{ }^{\circ} \mathrm{C}$ and moved to room temperature and stirred for $30 \mathrm{~min}$. Then, $\mathrm{K}_{2} \mathrm{CO}_{3}\left(172 \mathrm{mg}, 1.25 \mathrm{mmol}\right.$ ) was added and stirred at $80{ }^{\circ} \mathrm{C}$ (oil bath temperature) for $10 \mathrm{~h}$. After completion of the reaction (monitored by TLC), the reaction mixture was concentrated under reduced pressure. The crude product was purified by column chromatography on silica gel (EtOAc-hexane) to afford the Ethyl 4-benzyl-7methylthieno[2,3-e]benzofuran-8-carboxylate 3f, $144 \mathrm{mg}$, $82 \%$ yield, Brown solid; $R_{f}=0.5$ (hexane: EtOAc = 9:1); mp 106-108 ${ }^{\circ} \mathrm{C} ;{ }^{1} \mathrm{H}$ NMR $\left(400 \mathrm{MHz}, \mathrm{CDCl}_{3}\right) \delta 7.48(\mathrm{~d}, J=5.6 \mathrm{~Hz}$, 1H), $7.43(\mathrm{~d}, J=5.6 \mathrm{~Hz}, 1 \mathrm{H}), 7.30-7.26(\mathrm{~m}, 2 \mathrm{H}), 7.24-7.18(\mathrm{~m}, 4 \mathrm{H}), 4.52(\mathrm{q}, J=7.1 \mathrm{~Hz}$, 2H), $4.40(\mathrm{~s}, 2 \mathrm{H}), 2.79(\mathrm{~s}, 3 \mathrm{H}), 1.51(\mathrm{t}, J=7.1 \mathrm{~Hz}, 3 \mathrm{H}) ;{ }^{13} \mathrm{C} \mathrm{NMR}\left(100 \mathrm{MHz}, \mathrm{CDCl}_{3}\right) \delta$ $164.3,162.4,151.3,140.1,136.4,132.5,131.9,128.8,128.5,126.3,125.8,122.0,118.8$, 109.4, 109.1, 60.7, 40.1, 14.8, 14.6; IR (KBr): $v \max =1724,1272,1081,802,755 \mathrm{~cm}^{-1}$; HRMS (ESI): $m / z$ calcd for $\mathrm{C}_{21} \mathrm{H}_{19} \mathrm{O}_{3} \mathrm{~S}(\mathrm{M}+\mathrm{H})^{+}: 351.1055$, found: 351.1059 .

Ethyl 2-methyl-5-(4-(trifluoromethyl) benzyl) naphtho [2,1-b] furan-1-carboxylate (3g):

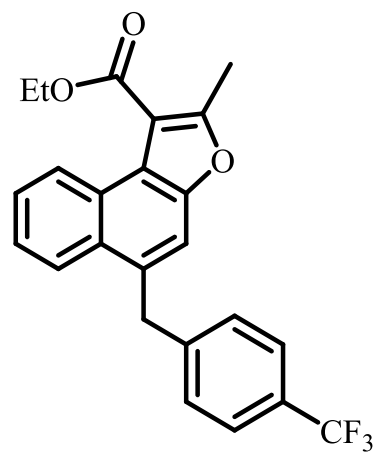

Following the general procedure,to the solution of 1-phenyl-5-(4-(trifluoromethyl)phenyl)penta2,4-diyn-1-ol $1 \mathbf{g}$ (150 mg, $0.5 \mathrm{mmol}$ ) and ethyl 3-oxobutanoate $2 \mathbf{a}(45 \mathrm{mg}, 0.35 \mathrm{mmol})$ in $3 \mathrm{~mL}$ of acetonitrile in a reaction vial was added $\mathrm{BF}_{3} \cdot \mathrm{Et}_{2} \mathrm{O}(7 \mathrm{mg}, 10 \mathrm{~mol} \%)$ at $0{ }^{\circ} \mathrm{C}$ and moved to room temperature and stirred for $30 \mathrm{~min}$. Then, $\mathrm{K}_{2} \mathrm{CO}_{3}(172 \mathrm{mg}, 1.25 \mathrm{mmol})$ was added and stirred at $80{ }^{\circ} \mathrm{C}$ (oil bath temperature) for $8 \mathrm{~h}$. After completion of the reaction (monitored by TLC), the reaction mixture was concentrated under reduced pressure. The crude product was purified by column chromatography on silica gel (EtOAc-hexane) to afford the Ethyl 2methyl-5-(4-(trifluoromethyl)benzyl)naphtho[2,1-b]furan-1-carboxylate $3 g 175 \mathrm{mg}, 85 \%$ yield, off-white solid; $R_{f}=0.8$ (hexane: EtOAc $=9: 1$ ); mp $108-110{ }^{\circ} \mathrm{C} ;{ }^{1} \mathrm{H}$ NMR $(500 \mathrm{MHz}$, $\left.\mathrm{CDCl}_{3}\right) \delta 9.25(\mathrm{dd}, J=8.5,0.7 \mathrm{~Hz}, 1 \mathrm{H}), 7.98(\mathrm{~d}, J=8.2 \mathrm{~Hz}, 1 \mathrm{H}), 7.60-7.57(\mathrm{~m}, 1 \mathrm{H}), 7.53$ $(\mathrm{d}, J=8.1 \mathrm{~Hz}, 2 \mathrm{H}), 7.49-7.45(\mathrm{~m}, 1 \mathrm{H}), 7.30$ (d, $J=8.0 \mathrm{~Hz}, 2 \mathrm{H}), 7.26(\mathrm{~s}, 1 \mathrm{H}), 4.55(\mathrm{~s}, 2 \mathrm{H})$, $4.50(\mathrm{q}, J=7.1 \mathrm{~Hz}, 2 \mathrm{H}), 2.77(\mathrm{~s}, 3 \mathrm{H}), 1.48(\mathrm{t}, J=7.1 \mathrm{~Hz}, 3 \mathrm{H}) ;{ }^{13} \mathrm{C} \mathrm{NMR}\left(100 \mathrm{MHz}, \mathrm{CDCl}_{3}\right)$ $\delta 165.1,161.2,151.1,144.4,133.8,129.7,129.0,128.4,126.8,126.1,125.5,125.4,125.0$, 
124.7, 124.3 (q, J = 271.8 Hz), 119.8, 113.3, 111.8, 60.9, 39.3, 15.3, 14.4; ${ }^{19} \mathrm{~F}$ NMR (377 $\left.\mathrm{MHz}, \mathrm{CDCl}_{3}\right) \delta-63.02$; IR (KBr): $v_{\max }=1328,1174,1130 \mathrm{~cm}^{-1} ;$ HRMS (ESI): $\mathrm{m} / z$ calcd for $\mathrm{C}_{24} \mathrm{H}_{20} \mathrm{O}_{3} \mathrm{~F}_{3}(\mathrm{M}+\mathrm{H})^{+}$: 413.1365, found: 413.1369 .

Ethyl 2-methyl-5-((trimethylsilyl)methyl) naphtho[2,1-b] furan-1-carboxylate (3h):<smiles>CCOC(=O)c1c(C)oc2cc(C[Si]C)c3ccccc3c12</smiles>

Following the general procedure,to the solution of 1-phenyl-5-(trimethylsilyl)penta-2,4-diyn-1-ol 1h (114 mg, $0.5 \mathrm{mmol})$ and ethyl 3-oxobutanoate $\mathbf{2 a}(45 \mathrm{mg}, 0.35 \mathrm{mmol})$ in $3 \mathrm{~mL}$ of acetonitrile in a reaction vial was added $\mathrm{BF}_{3} \cdot \mathrm{Et}_{2} \mathrm{O}(7 \mathrm{mg}, 10 \mathrm{~mol} \%)$ at $0{ }^{\circ} \mathrm{C}$ and moved to room temperature and stirred for $30 \mathrm{~min}$. Then, $\mathrm{K}_{2} \mathrm{CO}_{3}(172 \mathrm{mg}, 1.25 \mathrm{mmol})$ was added and stirred at $80{ }^{\circ} \mathrm{C}$ (oil bath temperature) for $7 \mathrm{~h}$. After completion of the reaction (monitored by TLC), the reaction mixture was concentrated under reduced pressure. The crude product was purified by column chromatography on silica gel (EtOAc-hexane) to afford the Ethyl 2methyl-5-((trimethylsilyl)methyl)naphtho[2,1-b]furan-1-carboxylate 3h, $146 \mathrm{mg}, 86 \%$ yield, off-white solid; $R_{f}=0.9$ (hexane: EtOAc $\left.=9: 1\right) ; \mathrm{mp} 184-186{ }^{\circ} \mathrm{C} ;{ }^{1} \mathrm{H}$ NMR $\left(400 \mathrm{MHz}, \mathrm{CDCl}_{3}\right)$ $7.25-7.19$ (m, 2H), $7.19-7.12(\mathrm{~m}, 3 \mathrm{H}), 3.97$ (q, J = 7.1 Hz, 2H), $3.33(\mathrm{~s}, 2 \mathrm{H}), 2.47$ (s, 3H), $0.94(\mathrm{t}, J=7.1 \mathrm{~Hz}, 3 \mathrm{H}), 0.01-0.02(\mathrm{~m}, 9 \mathrm{H}) ;{ }^{13} \mathrm{C} \mathrm{NMR}\left(100 \mathrm{MHz}, \mathrm{CDCl}_{3}\right) \delta 164.1,158.5$, 144.5, 132.4, 130.1 (2C), 127.8 (2C), 127.2, 122.4, 113.8, 101.3, 85.9, 59.9, 18.0, 14.2, 14.0, 0.03; IR (KBr): vmax $=1717,1091,851,764 \mathrm{~cm}^{-1}$; HRMS (ESI): $\mathrm{m} / z$ calcd for $\mathrm{C}_{20} \mathrm{H}_{25} \mathrm{O}_{3} \mathrm{Si}$ $(\mathrm{M}+\mathrm{H})^{+}: 341.1573$, found: 341.1567.

\section{Methyl 5-benzyl-2-methylnaphtho[2,1-b]furan-1-carboxylate (3i):}

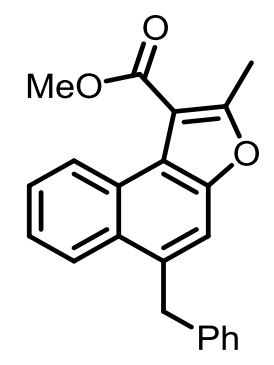

Following the general procedure,to the solution of 1,5-diphenylpenta-2,4-diyn-1-ol 1a (116 mg, $0.5 \mathrm{mmol}$ ) and methyl 3-oxobutanoate $2 \mathbf{b}(40 \mathrm{mg}, 0.35 \mathrm{mmol})$ in $3 \mathrm{~mL}$ of acetonitrile in a reaction vial was added $\mathrm{BF}_{3} \cdot \mathrm{Et}_{2} \mathrm{O}(7 \mathrm{mg}, 10 \mathrm{~mol} \%)$ at $0{ }^{\circ} \mathrm{C}$ and moved to room temperature 
and stirred for $30 \mathrm{~min}$. Then, $\mathrm{K}_{2} \mathrm{CO}_{3}(172 \mathrm{mg}, 1.25 \mathrm{mmol})$ was added and stirred at $80{ }^{\circ} \mathrm{C}$ (oil bath temperature) for $9 \mathrm{~h}$. After completion of the reaction (monitored by TLC), the reaction mixture was concentrated under reduced pressure. The crude product was purified by column chromatography on silica gel (EtOAc-hexane) to afford the Methyl 5-benzyl-2methylnaphtho[2,1-b]furan-1-carboxylate $3 \mathbf{i}, 135 \mathrm{mg}, 82 \%$ yield , off-white solid; $R_{f}=0.6$ (hexane: EtOAc = 9:1); $\mathrm{mp} 134-136{ }^{\circ} \mathrm{C} ;{ }^{1} \mathrm{H}$ NMR $(400 \mathrm{MHz}, \mathrm{CDCl} 3) \delta 9.20(\mathrm{dd}, \mathrm{J}=8.5,0.8$ $\mathrm{Hz}, 1 \mathrm{H}), 8.08(\mathrm{~d}, \mathrm{~J}=8.4 \mathrm{~Hz}, 1 \mathrm{H}), 7.61-7.55(\mathrm{~m}, 1 \mathrm{H}), 7.50-7.44(\mathrm{~m}, 1 \mathrm{H}), 7.37$ (s, 1H), 7.31 - $7.26(\mathrm{~m}, 2 \mathrm{H}), 7.24-7.18(\mathrm{~m}, \mathrm{~J}=7.1,4.9 \mathrm{~Hz}, 3 \mathrm{H}), 4.51$ (s, 2H), 4.02 (s, 3H), 2.75 (s, 3H); ${ }^{13} \mathrm{C}$ NMR $\left(100 \mathrm{MHz}, \mathrm{CDCl}_{3}\right) \delta 165.6,161.2,151.3,140.1,135.2,130,128.9,128.6,128.3$, $126.5,126.3,126.1,124.9,124.8,119.3,113.1,111.5,51.8,39.4,15.3$; IR (KBr): vmax = 1717, 1115, 1079, 803, 756, $704 \mathrm{~cm}^{-1}$; HRMS (ESI): $\mathrm{m} / z$ calcd for $\mathrm{C}_{22} \mathrm{H}_{19} \mathrm{O}_{3}(\mathrm{M}+\mathrm{H})^{+}$: 331.1334 found: 331.1335 .

\section{Allyl 5-benzyl-2-methylnaphtho[2,1-b]furan-1-carboxylate (3j):}

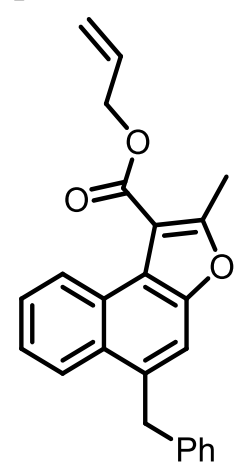

Following the general procedure, to the solution of 1,5-diphenylpenta-2,4-diyn-1-ol 1a (116 mg, $0.5 \mathrm{mmol}$ ) and allyl 3-oxobutanoate $2 \mathrm{c}(50 \mathrm{mg}, 0.35 \mathrm{mmol})$ in in $3 \mathrm{~mL}$ of acetonitrile in a reaction vial was added $\mathrm{BF}_{3} \cdot \mathrm{Et}_{2} \mathrm{O}(7 \mathrm{mg}, 10 \mathrm{~mol} \%)$ at $0{ }^{\circ} \mathrm{C}$ and moved to room temperature and stirred for $30 \mathrm{~min}$. Then, $\mathrm{K}_{2} \mathrm{CO}_{3}\left(172 \mathrm{mg}, 1.25 \mathrm{mmol}\right.$ ) was added and stirred at $80{ }^{\circ} \mathrm{C}$ (oil bath temperature) for $8 \mathrm{~h}$. After completion of the reaction (monitored by TLC), the reaction mixture was concentrated under reduced pressure. The crude product was purified by column chromatography on silica gel (EtOAc-hexane) to afford the Allyl 5-benzyl-2methylnaphtho[2,1-b]furan-1-carboxylate $3 \mathbf{3}, 145 \mathrm{mg}, 81 \%$ yield, off-white solid; $R_{f}=0.9$ (hexane: EtOAc = 9:1); $\mathrm{mp} 103-105{ }^{\circ} \mathrm{C} ;{ }^{1} \mathrm{H}$ NMR $\left(400 \mathrm{MHz}, \mathrm{CDCl}_{3}\right) \delta 9.22(\mathrm{dd}, J=8.5,0.8$ $\mathrm{Hz}, 1 \mathrm{H}), 8.08(\mathrm{dd}, J=8.5,0.7 \mathrm{~Hz}, 1 \mathrm{H}), 7.59-7.54(\mathrm{~m}, J=8.4,6.8,1.3 \mathrm{~Hz}, 1 \mathrm{H}), 7.49-7.44$ $(\mathrm{m}, J=8.3,6.9,1.4 \mathrm{~Hz}, 1 \mathrm{H}), 7.37$ (s, 1H), $7.31-7.26(\mathrm{~m}, 2 \mathrm{H}), 7.23-7.19(\mathrm{~m}, 3 \mathrm{H}), 6.19-$ $6.06(\mathrm{~m}, 1 \mathrm{H}), 5.47(\mathrm{dq}, J=17.2,1.5 \mathrm{~Hz}, 1 \mathrm{H}), 5.34(\mathrm{dq}, J=10.4,1.2 \mathrm{~Hz}, 1 \mathrm{H}), 4.95(\mathrm{t}, J=1.3$ $\mathrm{Hz}, 1 \mathrm{H}), 4.93(\mathrm{t}, J=1.3 \mathrm{~Hz}, 1 \mathrm{H}), 4.51(\mathrm{~s}, 2 \mathrm{H}), 2.77(\mathrm{~s}, 3 \mathrm{H}) ;{ }^{13} \mathrm{C} \mathrm{NMR}\left(100 \mathrm{MHz}, \mathrm{CDCl}_{3}\right) \delta$ $164.8,161.3,151.4,140.1,135.2$, 132.2, 129.9, 128.9, 128.6, 128.3, 126.7, 126.3, 126.1, 
124.9, 124.8, 119.4, 118.9, 113.1, 111.6, 65.6, 39.5, 15.4; IR (KBr): $v \max =1672,1384,762$, $714 \mathrm{~cm}^{-1}$; HRMS (ESI): $\mathrm{m} / z$ calcd for $\mathrm{C}_{24} \mathrm{H}_{21} \mathrm{O}_{3}(\mathrm{M}+\mathrm{H})^{+}: 357.1491$, found: 357.1497.

Ethyl 5-benzyl-2-(p-tolyl)naphtho[2,1-b]furan-1-carboxylate (3k):

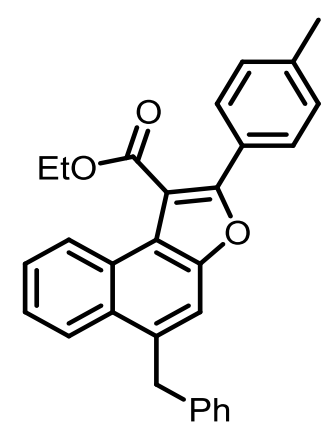

Following the general procedure,to the solution of 1,5-diphenylpenta-2,4-diyn-1-ol 1a (116 mg, $0.5 \mathrm{mmol}$ ) and ethyl 3-oxo-3-(p-tolyl)propanoate $2 \mathbf{d}(72 \mathrm{mg}, 0.35 \mathrm{mmol})$ in in $3 \mathrm{~mL}$ of acetonitrile in a reaction vial was added $\mathrm{BF}_{3} \cdot \mathrm{Et}_{2} \mathrm{O}(7 \mathrm{mg}, 10 \mathrm{~mol} \%)$ at $0{ }^{\circ} \mathrm{C}$ and moved to room temperature and stirred for $30 \mathrm{~min}$. Then, $\mathrm{K}_{2} \mathrm{CO}_{3}(172 \mathrm{mg}, 1.25 \mathrm{mmol})$ was added and stirred at $80{ }^{\circ} \mathrm{C}$ (oil bath temperature) for $9 \mathrm{~h}$. After completion of the reaction (monitored by TLC), the reaction mixture was concentrated under reduced pressure. The crude product was purified by column chromatography on silica gel (EtOAc-hexane) to afford the Ethyl 5benzyl-2-(p-tolyl)naphtho[2,1-b]furan-1-carboxylate 3k, $169 \mathrm{mg}, 80 \%$ yield, off-white solid; $R_{f}=0.5$ (hexane: EtOAc $\left.=9: 1\right) ; \operatorname{mp~} 110-112{ }^{\circ} \mathrm{C} ;{ }^{1} \mathrm{H} \mathrm{NMR}\left(300 \mathrm{MHz}, \mathrm{CDCl}_{3}\right) \delta 8.59(\mathrm{~d}, J=$ $8.4 \mathrm{~Hz}, 1 \mathrm{H}), 8.10(\mathrm{~d}, J=8.3 \mathrm{~Hz}, 1 \mathrm{H}), 7.71(\mathrm{~d}, J=8.1 \mathrm{~Hz}, 2 \mathrm{H}), 7.61-7.55(\mathrm{~m}, 1 \mathrm{H}), 7.51-$ $7.45(\mathrm{~m}, 2 \mathrm{H}), 7.30(\mathrm{t}, J=7.2 \mathrm{~Hz}, 4 \mathrm{H}), 7.23(\mathrm{~d}, J=6.5 \mathrm{~Hz}, 3 \mathrm{H}), 4.54(\mathrm{~s}, 2 \mathrm{H}), 4.53-4.45(\mathrm{~m}$, 2H), $2.43(\mathrm{~s}, 3 \mathrm{H}), 1.35(\mathrm{t}, J=7.1 \mathrm{~Hz}, 3 \mathrm{H}) ;{ }^{13} \mathrm{C} \mathrm{NMR}\left(100 \mathrm{MHz}, \mathrm{CDCl}_{3}\right) \delta 166.5,155.9$, 151.5, 140.1, 139.6, 135.8, 129.9, 129.1, 128.9 (2C), 128.6, 128.0, 127.8, 127.2, 126.3, 125.3, 124.9, 124.9, 120.1, 113.4, 111.0, 61.6, 39.6, 21.5, 14.0; IR (KBr): $\max =1722,1099,1032$, $759 \mathrm{~cm}^{-1}$; HRMS (ESI): $\mathrm{m} / z$ calcd for $\mathrm{C}_{29} \mathrm{H}_{25} \mathrm{O}_{3}(\mathrm{M}+\mathrm{H})^{+}: 421.1804$, found: 421.1801 .

\section{1-(5-Benzyl-2-methylnaphtho[2,1-b]furan-1-yl) ethan-1-one (31):}

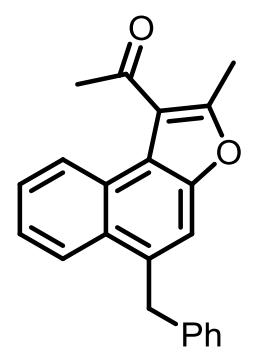

Following the general procedure, to the solution of 1,5-diphenylpenta-2,4-diyn-1-ol 1a (116 mg, $0.5 \mathrm{mmol}$ ) and pentane-2,4-dione $2 \mathrm{e}(35 \mathrm{mg}, 0.35 \mathrm{mmol})$ in in $3 \mathrm{~mL}$ of acetonitrile in a reaction vial was added $\mathrm{BF}_{3} \cdot \mathrm{Et}_{2} \mathrm{O}(7 \mathrm{mg}, 10 \mathrm{~mol} \%)$ at $0{ }^{\circ} \mathrm{C}$ and moved to room temperature and stirred 
for $30 \mathrm{~min}$. Then, $\mathrm{K}_{2} \mathrm{CO}_{3}(172 \mathrm{mg}, 1.25 \mathrm{mmol})$ was added and stirred at $80{ }^{\circ} \mathrm{C}$ (oil bath temperature) for $10 \mathrm{~h}$. After completion of the reaction (monitored by TLC), the reaction mixture was concentrated under reduced pressure. The crude product was purified by column chromatography on silica gel (EtOAc-hexane) to afford the 1-(5-benzyl-2methylnaphtho[2,1-b]furan-1-yl)ethan-1-one 3l, $134 \mathrm{mg}, 85 \%$ yield, off-white solid; $R_{f}=0.8$ (hexane: EtOAc = 9:1); mp 138-140 ${ }^{\circ} \mathrm{C} ;{ }^{1} \mathrm{H}$ NMR $\left(400 \mathrm{MHz}, \mathrm{CDCl}_{3}\right) \delta 8.65(\mathrm{dd}, J=8.4,0.8$ $\mathrm{Hz}, 1 \mathrm{H}), 8.08(\mathrm{~d}, J=7.8 \mathrm{~Hz}, 1 \mathrm{H}), 7.58-7.53(\mathrm{~m}, J=8.4,6.9,1.3 \mathrm{~Hz}, 1 \mathrm{H}), 7.48-7.44(\mathrm{~m}, J$ $=8.3,6.9,1.4 \mathrm{~Hz}, 1 \mathrm{H}), 7.37(\mathrm{~s}, 1 \mathrm{H}), 7.31-7.26(\mathrm{~m}, 2 \mathrm{H}), 7.23-7.18(\mathrm{~m}, 3 \mathrm{H}), 4.50(\mathrm{~s}, 2 \mathrm{H})$,

$2.72(\mathrm{~s}, 3 \mathrm{H}), 2.71(\mathrm{~s}, 3 \mathrm{H}) ;{ }^{13} \mathrm{C}$ NMR $\left(100 \mathrm{MHz}, \mathrm{CDCl}_{3}\right) \delta 197.3,157.5,151.3,140.1,135.4$, 129.9, 128.8, 128.5, 128.2, 126.3, 126.1, 126.0, 125.1, 124.8, 121.8, 119.3, 113.1, 39.5, 31.9, 15.2; IR (KBr): vmax $=1717,1435,1116,762 \mathrm{~cm}^{-1}$; HRMS (ESI): $\mathrm{m} / z$ calcd for $\mathrm{C}_{22} \mathrm{H}_{19} \mathrm{O}_{2}$ $(\mathrm{M}+\mathrm{H})^{+}:$315.1385, found: 315.1382 .

\section{1-(5-Benzyl-7-methoxy-2-methylnaphtho[2,1-b]furan-1-yl)ethan-1-one (3m):}

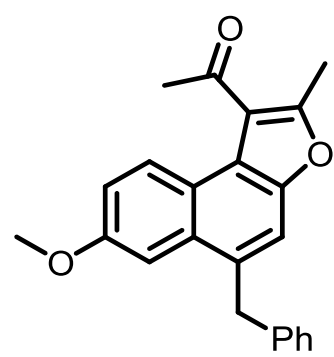

Following the general procedure, to the solution of 1-(4-methoxyphenyl)-5-phenylpenta-2,4-diyn1-ol $1 \mathbf{b}$ (131 mg, $0.5 \mathrm{mmol}$ ) and pentane-2,4-dione $2 \mathbf{e}(35 \mathrm{mg}, 0.35 \mathrm{mmol})$ in $3 \mathrm{~mL}$ of acetonitrile in a reaction vial was added $\mathrm{BF}_{3} \cdot \mathrm{Et}_{2} \mathrm{O}(7 \mathrm{mg}, 10 \mathrm{~mol} \mathrm{\%})$ at $0{ }^{\circ} \mathrm{C}$ and moved to room temperature and stirred for $30 \mathrm{~min}$. Then, $\mathrm{K}_{2} \mathrm{CO}_{3}(172 \mathrm{mg}, 1.25 \mathrm{mmol})$ was added and stirred at $80{ }^{\circ} \mathrm{C}$ (oil bath temperature) for $8 \mathrm{~h}$. After completion of the reaction (monitored by TLC), the reaction mixture was concentrated under reduced pressure. The crude product was purified by column chromatography on silica gel (EtOAc-hexane) to afford the 1-(5-benzyl7-methoxy-2-methylnaphtho[2,1-b]furan-1-yl)ethan-1-one 3m, $140 \mathrm{mg}, 81 \%$ yield, gray solid; $R_{f}=0.5$ (hexane: $\left.\mathrm{EtOAc}=9: 1\right) ; \mathrm{mp} 124-126{ }^{\circ} \mathrm{C} ;{ }^{1} \mathrm{H} \mathrm{NMR}\left(500 \mathrm{MHz}, \mathrm{CDCl}_{3}\right) \delta 8.67(\mathrm{~d}, J=$ $9.2 \mathrm{~Hz}, 1 \mathrm{H}), 7.37$ (s, 1H), 7.36 (d, J = 2.6 Hz, 1H), $7.30-7.26(\mathrm{~m}, 2 \mathrm{H}), 7.23-7.19(\mathrm{~m}, 4 \mathrm{H})$, $4.44(\mathrm{~s}, 2 \mathrm{H}), 3.81(\mathrm{~s}, 3 \mathrm{H}), 2.73(\mathrm{~s}, 3 \mathrm{H}), 2.69(\mathrm{~s}, 3 \mathrm{H}) ;{ }^{13} \mathrm{C} \mathrm{NMR}\left(125 \mathrm{MHz}, \mathrm{CDCl}_{3}\right) \delta$ 197.0, $157.9,156.6,150.2$, 140.0, 134.2, 131.3, 128.8, 128.6, 127.7, 126.3, 123.1, 121.6, 119.5, 117.2, 113.3, 105.2, 55.2, 39.9, 31.9, 15.4; IR (KBr): $v \max =1669,1444,1369,1232,1030$, $757 \mathrm{~cm}^{-1}$; HRMS (ESI): $\mathrm{m} / z$ calcd for $\mathrm{C}_{23} \mathrm{H}_{21} \mathrm{O}_{3}(\mathrm{M}+\mathrm{H})^{+}: 345.1491$, found: 345.1491 .

\section{1-(5-Benzyl-2-methylphenanthro[3,4-b]furan-1-yl)ethan-1-one (3n):}




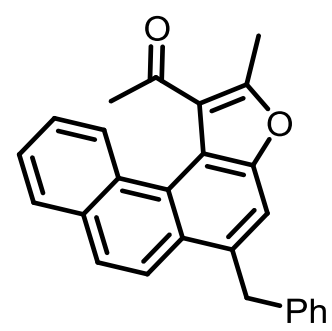

Following the general procedure, to the solution of 1-(naphthalen-1-yl)-5-phenylpenta-2,4-diyn-1ol $1 \mathbf{d}$ (141 mg, $0.5 \mathrm{mmol})$ and pentane-2,4-dione $2 \mathbf{e}(35 \mathrm{mg}, 0.35 \mathrm{mmol})$ in $3 \mathrm{~mL}$ of acetonitrile in a reaction vial was added $\mathrm{BF}_{3} \cdot \mathrm{Et}_{2} \mathrm{O}(7 \mathrm{mg}, 10 \mathrm{~mol} \%)$ at $0{ }^{\circ} \mathrm{C}$ and moved to room temperature and stirred for $30 \mathrm{~min}$. Then, $\mathrm{K}_{2} \mathrm{CO}_{3}(172 \mathrm{mg}, 1.25 \mathrm{mmol})$ was added and stirred at $80{ }^{\circ} \mathrm{C}$ (oil bath temperature) for $8 \mathrm{~h}$. After completion of the reaction (monitored by TLC), the reaction mixture was concentrated under reduced pressure. The crude product was purified by column chromatography on silica gel (EtOAc-hexane) to afford the corresponding product 1-(5-benzyl-2-methylphenanthro[3,4-b]furan-1-yl)ethan-1-one 3n, 140 $\mathrm{mg}, 77 \%$ yield, gray solid; $R_{f}=0.5$ (hexane: EtOAc $=9: 1$ ); $\mathrm{mp} 124-126{ }^{\circ} \mathrm{C} ;{ }^{1} \mathrm{H}$ NMR $(400$ $\left.\mathrm{MHz}, \mathrm{CDCl}_{3}\right) \delta 8.41-8.37(\mathrm{~m}, 1 \mathrm{H}), 8.03(\mathrm{~d}, J=9.1 \mathrm{~Hz}, 1 \mathrm{H}), 7.93-7.90(\mathrm{~m}, 1 \mathrm{H}), 7.75(\mathrm{~d}, J$ $=9.0 \mathrm{~Hz}, 1 \mathrm{H}), 7.59-7.55(\mathrm{~m}, 2 \mathrm{H}), 7.55(\mathrm{~s}, 1 \mathrm{H}), 7.32-7.28(\mathrm{~m}, J=9.3,5.4 \mathrm{~Hz}, 2 \mathrm{H}), 7.23$ $(\mathrm{d}, J=7.3 \mathrm{~Hz}, 3 \mathrm{H}), 4.58(\mathrm{~s}, 2 \mathrm{H}), 2.67$ (s, 3H), 2.07 (s, 3H); ${ }^{13} \mathrm{C} \mathrm{NMR}\left(100 \mathrm{MHz}, \mathrm{CDCl}_{3}\right) \delta$ 198.6, 157.4, 153.1, 140.2, 135.0, 132.2, 130.1, 128.8, 128.7, 128.1, 127.9, 126.8, 126.7, $126.4,125.7,125.6,123.3,122.5,118.7,113.3,39.7,32.2$, 13.6; IR $(\mathrm{KBr}): v \max =3784$, $3045,1684,1573,761 \mathrm{~cm}^{-1}$; HRMS (ESI): $\mathrm{m} / z$ calcd for $\mathrm{C}_{26} \mathrm{H}_{21} \mathrm{O}_{2}(\mathrm{M}+\mathrm{H})^{+}: 365.1542$, found: 365.1544 .

\section{5-Benzyl-9,10-dihydronaphtho[2,1-b] benzofuran-11(8H)-one (3o):}

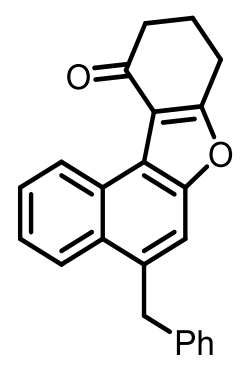

Following the general procedure,to the solution of 1,5-diphenylpenta-2,4-diyn-1-ol 1a (116 mg, $0.5 \mathrm{mmol}$ ) and cyclohexane-1,3-dione $2 \mathbf{f}(39 \mathrm{mg}, 0.35 \mathrm{mmol})$ in in $3 \mathrm{~mL}$ of acetonitrile in a reaction vial was added $\mathrm{BF}_{3} \cdot \mathrm{Et}_{2} \mathrm{O}\left(7 \mathrm{mg}, 10 \mathrm{~mol} \%\right.$ ) at $0{ }^{\circ} \mathrm{C}$ and moved to room temperature and stirred for $30 \mathrm{~min}$. Then, $\mathrm{K}_{2} \mathrm{CO}_{3}\left(172 \mathrm{mg}, 1.25 \mathrm{mmol}\right.$ ) was added and stirred at $80{ }^{\circ} \mathrm{C}$ (oil bath temperature) for $11 \mathrm{~h}$. After completion of the reaction (monitored by TLC), the reaction mixture was concentrated under reduced pressure. The crude product was purified by column 
chromatography on silica gel (EtOAc-hexane) to afford the 5-benzyl-9,10dihydronaphtho[2,1-b]benzofuran-11(8H)-one 3o, $145 \mathrm{mg}, 89 \%$ yield, off-white solid; $R_{f}=$ 0.6 (hexane: EtOAc $=9: 1) ; \mathrm{mp} 105-107{ }^{\circ} \mathrm{C} ;{ }^{1} \mathrm{H}$ NMR $\left(400 \mathrm{MHz}, \mathrm{CDCl}_{3}\right) \delta 9.69$ (d, $J=8.3$ $\mathrm{Hz}, 1 \mathrm{H}), 8.07$ (d, $J=8.4 \mathrm{~Hz}, 1 \mathrm{H}), 7.64$ (t, $J=7.5 \mathrm{~Hz}, 1 \mathrm{H}), 7.50$ (t, $J=7.5 \mathrm{~Hz}, 1 \mathrm{H}), 7.40$ (s, 1H), $7.32-7.25(\mathrm{~m}, 2 \mathrm{H}), 7.24-7.15(\mathrm{~m}, 3 \mathrm{H}), 4.51(\mathrm{~s}, 2 \mathrm{H}), 3.10(\mathrm{t}, J=6.1 \mathrm{~Hz}, 2 \mathrm{H}), 2.75-$ $2.69(\mathrm{~m}, 2 \mathrm{H}), 2.35-2.22(\mathrm{~m}, 2 \mathrm{H}) ;{ }^{13} \mathrm{C} \mathrm{NMR}\left(100 \mathrm{MHz}, \mathrm{CDCl}_{3}\right) \delta 194.2,169.8,152.1,140.0$, $136.0,129.9,128.8,128.7,128.7,128.6,126.4,126.3,125.3,124.5,118.9,118.6,112.9$, 39.4, 39.1, 24.4, 22.3; IR (KBr): vmax = 1671, 1438, 1375, 1008, $761 \mathrm{~cm}^{-1}$; HRMS (ESI): $\mathrm{m} / z$ calcd for $\mathrm{C}_{23} \mathrm{H}_{19} \mathrm{O}_{2}(\mathrm{M}+\mathrm{H})^{+}: 327.1385$, found: 327.1383 .

\section{5-Benzyl-3-methoxy-9,10-dihydronaphtho[2,1-b] benzofuran-11(8H)-one (3p):}

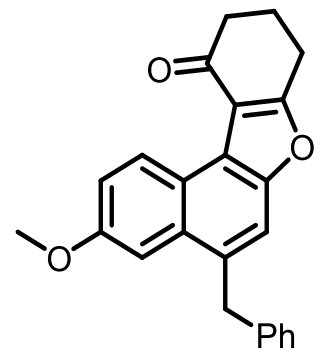

Following the general procedure, to the solution of 1-(4-methoxyphenyl)-5-phenylpenta-2,4-diyn1-ol 1a (131 mg, $0.5 \mathrm{mmol})$ and cyclohexane-1,3-dione $2 \mathbf{f}(39 \mathrm{mg}, 0.35 \mathrm{mmol})$ in $3 \mathrm{~mL}$ of acetonitrile in a reaction vial was added $\mathrm{BF}_{3} \cdot \mathrm{Et}_{2} \mathrm{O}(7 \mathrm{mg}, 10 \mathrm{~mol} \%)$ at $0{ }^{\circ} \mathrm{C}$ and moved to room temperature and stirred for $30 \mathrm{~min}$. Then, $\mathrm{K}_{2} \mathrm{CO}_{3}(172 \mathrm{mg}, 1.25 \mathrm{mmol})$ was added and stirred at $80{ }^{\circ} \mathrm{C}$ (oil bath temperature) for $8 \mathrm{~h}$. After completion of the reaction (monitored by TLC), the reaction mixture was concentrated under reduced pressure. The crude product was purified by column chromatography on silica gel (EtOAc-hexane) to afford the 5-benzyl-3methoxy-9,10-dihydronaphtho[2,1-b]benzofuran-11(8H)-one 3p, $134 \mathrm{mg}$, 75\% yield, offwhite solid; $R_{f}=0.5$ (hexane: EtOAc $=9: 1$ ); mp 112-114 ${ }^{\circ} \mathrm{C} ;{ }^{1} \mathrm{H}$ NMR $\left(400 \mathrm{MHz}, \mathrm{CDCl}_{3}\right) \delta$ $9.61(\mathrm{~d}, J=9.2 \mathrm{~Hz}, 1 \mathrm{H}), 7.40$ (s, 1H), 7.34 (d, $J=2.5 \mathrm{~Hz}, 1 \mathrm{H}), 7.31-7.26$ (m, 3H), 7.24 $7.18(\mathrm{~m}, 3 \mathrm{H}), 4.45$ (s, 2H), $3.82(\mathrm{~s}, 3 \mathrm{H}), 3.10(\mathrm{t}, J=6.3 \mathrm{~Hz}, 2 \mathrm{H}), 2.75-2.68(\mathrm{~m}, 2 \mathrm{H}), 2.29$ $(\mathrm{dd}, J=12.9,6.4 \mathrm{~Hz}, 2 \mathrm{H}) ;{ }^{13} \mathrm{C} \mathrm{NMR}\left(101 \mathrm{MHz}, \mathrm{CDCl}_{3}\right) \delta 194.3,169.9,157.0,151.0,140.0$, 134.7, 131.3, 130.3, 128.8 (2C), 128.6, 126.3, 123.6, 118.7, 117.5, 113.3, 104.6, 55.2, 39.9, 39.1, 24.4, 22.3; IR (KBr): vmax $=1670,1528,1376,1235,760 \mathrm{~cm}^{-1} ; \mathrm{HRMS}(\mathrm{ESI}): \mathrm{m} / z$ calcd for $\mathrm{C}_{24} \mathrm{H}_{21} \mathrm{O}_{3}(\mathrm{M}+\mathrm{H})^{+}:$357.1491, found: 357.1488 .

Ethyl 5-benzyl-2-methyl-6-tosyl-6H-furo[2,3-c] carbazole-1-carboxylate (3q): 


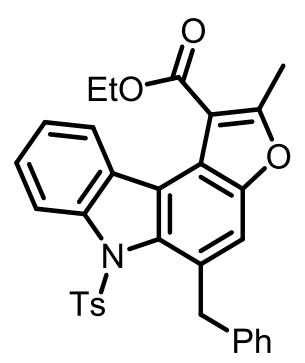

Following the general procedure, to the solution 5-phenyl-1-(1-tosyl-1H-indol-3-yl)penta-2,4diyn-1-ol 1i (212 mg, $0.5 \mathrm{mmol}$ ) and ethyl 3-oxobutanoate $\mathbf{2 a}$ ( $45 \mathrm{mg}, 0.35 \mathrm{mmol}$ ) in $3 \mathrm{~mL}$ of acetonitrile in a reaction vial was added $\mathrm{BF}_{3} \cdot \mathrm{Et}_{2} \mathrm{O}(7 \mathrm{mg}, 10 \mathrm{~mol} \%)$ at $0{ }^{\circ} \mathrm{C}$ and moved to room temperature and stirred for $30 \mathrm{~min}$. Then, $\mathrm{K}_{2} \mathrm{CO}_{3}(172 \mathrm{mg}, 1.25 \mathrm{mmol})$ was added and stirred at $80{ }^{\circ} \mathrm{C}$ (oil bath temperature) for $8 \mathrm{~h}$. After completion of the reaction (monitored by TLC), the reaction mixture was concentrated under reduced pressure. The crude product was purified by column chromatography on silica gel (EtOAc-hexane) to afford the Ethyl 5benzyl-2-methyl-6-tosyl-6H-furo[2,3-c]carbazole-1-carboxylate 3q, $240 \mathrm{mg}, 89 \%$ yield, brown solid; $R_{f}=0.5$ (hexane: EtOAc $\left.=9: 1\right) ; \mathrm{mp} \mathrm{143-145}{ }^{\circ} \mathrm{C} ;{ }^{1} \mathrm{H}$ NMR $\left(400 \mathrm{MHz}, \mathrm{CDCl}_{3}\right) \delta$ $8.20(\mathrm{~d}, J=8.1 \mathrm{~Hz}, 1 \mathrm{H}), 7.55(\mathrm{~d}, J=7.8 \mathrm{~Hz}, 1 \mathrm{H}), 7.39-7.35(\mathrm{~m}, 1 \mathrm{H}), 7.28$ (d, $J=8.5 \mathrm{~Hz}$, 2H), 7.27-7.25 (m, 1H), $7.20(\mathrm{t}, J=6.3 \mathrm{~Hz}, 3 \mathrm{H}), 7.13(\mathrm{~s}, 1 \mathrm{H}), 6.93(\mathrm{~d}, J=8.3 \mathrm{~Hz}, 2 \mathrm{H}), 6.80$ $(\mathrm{d}, J=8.2 \mathrm{~Hz}, 2 \mathrm{H}), 4.80$ (s, 2H), 4.34 (q, $J=7.1 \mathrm{~Hz}, 2 \mathrm{H}), 2.66$ (s, 3H), 2.16 (s, 3H), 1.13 (t, $J$ $=7.1 \mathrm{~Hz}, 3 \mathrm{H}) ;{ }^{13} \mathrm{C} \mathrm{NMR}\left(100 \mathrm{MHz}, \mathrm{CDCl}_{3}\right) \delta 164.7,162.4,153.1,144.1,142.6,141.5,138.0$, $132.7,130.9$, 130.7, 129.7, 128.4, 128.3, 127.4, 126.4, 126.1, 125.3, 124.7, 123.9, 119.9, 117.3, 111.8, 110.6, 60.8, 39.7, 21.4, 14.3, 13.9; IR (KBr): vmax = 1719, 1377, 1180, 1090, $762,667 \mathrm{~cm}^{-1}$; HRMS (ESI): $\mathrm{m} / z$ calcd for $\mathrm{C}_{32} \mathrm{H}_{27} \mathrm{NO}_{5} \mathrm{SNa}(\mathrm{M}+\mathrm{Na})^{+}: 560.1508$, found: 560.1515 .

\section{Methyl 5-benzyl-2-methyl-6-tosyl-6H-furo[2,3-c]carbazole-1-carboxylate (3r):}

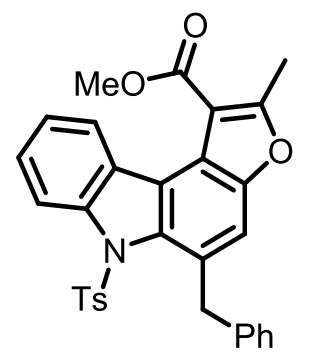

Following the general procedure,to the solution of 5-phenyl-1-(1-tosyl-1H-indol-3-yl)penta-2,4diyn-1-ol 1i (212 mg, $0.5 \mathrm{mmol}$ ) and methyl 3-oxobutanoate $\mathbf{2 b}$ (40 mg, $0.35 \mathrm{mmol})$ in $3 \mathrm{~mL}$ of acetonitrile in a reaction vial was added $\mathrm{BF}_{3} \cdot \mathrm{Et}_{2} \mathrm{O}(7 \mathrm{mg}, 10 \mathrm{~mol} \%)$ at $0{ }^{\circ} \mathrm{C}$ and moved to room temperature and stirred for $30 \mathrm{~min}$. Then, $\mathrm{K}_{2} \mathrm{CO}_{3}(172 \mathrm{mg}, 1.25 \mathrm{mmol})$ was added and 
stirred at $80{ }^{\circ} \mathrm{C}$ (oil bath temperature) for $10 \mathrm{~h}$. After completion of the reaction (monitored by TLC), the reaction mixture was concentrated under reduced pressure. The crude product was purified by column chromatography on silica gel (EtOAc-hexane) to afford the Methyl 5benzyl-2-methyl-6-tosyl-6H-furo[2,3-c]carbazole-1-carboxylate 3r, 225 mg, $86 \%$ yield, offwhite solid; $R_{f}=0.6$ (hexane: EtOAc $=9: 1$ ); mp 192-194 ${ }^{\circ} \mathrm{C} ;{ }^{1} \mathrm{H}$ NMR $\left(500 \mathrm{MHz}, \mathrm{CDCl}_{3}\right) \delta$ $8.19(\mathrm{~d}, J=8.1 \mathrm{~Hz}, 1 \mathrm{H}), 7.53(\mathrm{~d}, J=7.4 \mathrm{~Hz}, 1 \mathrm{H}), 7.40-7.35(\mathrm{~m}, 1 \mathrm{H}), 7.30-7.27(\mathrm{~m}, 2 \mathrm{H})$, $7.26-7.24(\mathrm{~m}, 1 \mathrm{H}), 7.23-7.17(\mathrm{~m}, 3 \mathrm{H}), 7.14(\mathrm{~s}, 1 \mathrm{H}), 6.92-6.86(\mathrm{~m}, 2 \mathrm{H}), 6.79(\mathrm{~d}, J=8.0$ $\mathrm{Hz}, 2 \mathrm{H}), 4.79$ (s, 2H), $3.84(\mathrm{~s}, 3 \mathrm{H}), 2.66(\mathrm{~s}, 3 \mathrm{H}), 2.17(\mathrm{~s}, 3 \mathrm{H}) ;{ }^{13} \mathrm{C} \mathrm{NMR}\left(125 \mathrm{MHz}, \mathrm{CDCl}_{3}\right) \delta$ $165.0,162.5,153.1,144.1,142.6,141.5,138.1$, 132.7, 130.8, 130.6, 129.6, 128.4, 128.2, 127.4, 126.4, 126.1, 125.5, 124.8, 123.8, 120.0, 117.3, 111.8, 110.3, 51.5, 39.6, 21.4, 14.4; IR $(\mathrm{KBr}): \operatorname{vmax}=1723,1451,1377,1181,763 \mathrm{~cm}^{-1}$; HRMS (ESI): $\mathrm{m} / z$ calcd for $\mathrm{C}_{31} \mathrm{H}_{25} \mathrm{NO}_{5} \mathrm{NaS}(\mathrm{M}+\mathrm{Na})^{+}:$546.1351, found: 546.1354 .

\section{1-(5-Benzyl-2-methyl-6-tosyl-6H-furo[2,3-c] carbazol-1-yl) ethan-1-one (3s):}

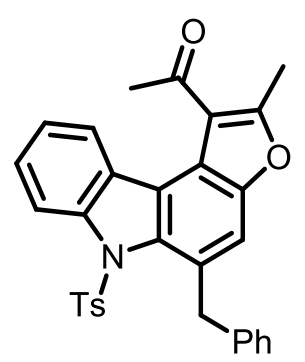

Following the general procedure, to the solution of 5-phenyl-1-(1-tosyl-1H-indol-3-yl)penta-2,4diyn-1-ol 1e (212 mg, $0.5 \mathrm{mmol})$ and pentane-2,4-dione $2 \mathbf{e}(35 \mathrm{mg}, 0.35 \mathrm{mmol})$ in $3 \mathrm{~mL}$ of acetonitrile in a reaction vial was added $\mathrm{BF}_{3} \cdot \mathrm{Et}_{2} \mathrm{O}(7 \mathrm{mg}, 10 \mathrm{~mol} \%)$ at $0{ }^{\circ} \mathrm{C}$ and moved to room temperature and stirred for $30 \mathrm{~min}$. Then, $\mathrm{K}_{2} \mathrm{CO}_{3}(172 \mathrm{mg}, 1.25 \mathrm{mmol})$ was added and stirred at $80{ }^{\circ} \mathrm{C}$ (oil bath temperature) for $9 \mathrm{~h}$. After completion of the reaction (monitored by TLC), the reaction mixture was concentrated under reduced pressure. The crude product was purified by column chromatography on silica gel (EtOAc-hexane) to afford the 1-(5-benzyl2-methyl-6-tosyl-6H-furo[2,3-c]carbazol-1-yl)ethan-1-one 3s, 215 mg, $85 \%$ yield, off-white solid; $R_{f}=0.5$ (hexane: EtOAc $=9: 1$ ); mp 190-192 ${ }^{\circ} \mathrm{C} ;{ }^{1} \mathrm{H}$ NMR $\left(400 \mathrm{MHz}, \mathrm{CDCl}_{3}\right) \delta 8.22$ $(\mathrm{d}, J=8.1 \mathrm{~Hz}, 1 \mathrm{H}), 7.45-7.37(\mathrm{~m}, 1 \mathrm{H}), 7.31-7.26(\mathrm{~m}, 3 \mathrm{H}), 7.22(\mathrm{t}, J=7.9 \mathrm{~Hz}, 4 \mathrm{H}), 7.13$ (s, 1H), $6.90(\mathrm{~d}, J=8.3 \mathrm{~Hz}, 2 \mathrm{H}), 6.80(\mathrm{~d}, J=8.2 \mathrm{~Hz}, 2 \mathrm{H}), 4.80(\mathrm{~s}, 2 \mathrm{H}), 2.57$ (s, 3H), 2.21 (s, 3H), $2.16(\mathrm{~s}, 3 \mathrm{H}) ;{ }^{13} \mathrm{C}$ NMR $\left(100 \mathrm{MHz}, \mathrm{CDCl}_{3}\right) \delta 196.9,158.9,153.0,144.2,142.8,141.2$, $137.9,133.0,130.4,130.3,129.6,128.5,128.2$, 127.5, 126.8, 126.2, 125.8, 124.3, 122.4, 120.6, 119.9, 117.2, 111.8, 39.4, 31.5, 21.3, 13.8; IR (KBr): $v_{\max }=1685,1376,1179,763$, $670 \mathrm{~cm}^{-1}$; HRMS (ESI): $\mathrm{m} / z$ calcd for $\mathrm{C}_{31} \mathrm{H}_{26} \mathrm{NO}_{4} \mathrm{~S}(\mathrm{M}+\mathrm{H})^{+}: 508.1583$, found: 508.1583. 
7-Benzyl-8-tosyl-1,2,4,8-tetrahydro-3H-benzofuro[2,3-c]carbazol-3-one (3t):

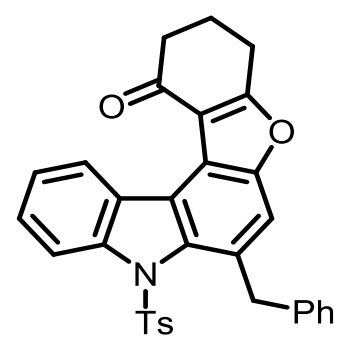

Following the general procedure, to the solution of 5-phenyl-1-(1-tosyl-1H-indol-3-yl)penta-2,4diyn-1-ol 1e (212 mg, $0.5 \mathrm{mmol})$ and cyclohexane-1,3-dione $2 \mathbf{f}(20 \mathrm{mg}, 0.35 \mathrm{mmol})$ in $3 \mathrm{~mL}$ of acetonitrile in a reaction vial was added $\mathrm{BF}_{3} \cdot \mathrm{Et}_{2} \mathrm{O}(7 \mathrm{mg}, 10 \mathrm{~mol} \%)$ at $0{ }^{\circ} \mathrm{C}$ and moved to room temperature and stirred for $30 \mathrm{~min}$. Then, $\mathrm{K}_{2} \mathrm{CO}_{3}(172 \mathrm{mg}, 1.25 \mathrm{mmol})$ was added and stirred at $80{ }^{\circ} \mathrm{C}$ (oil bath temperature) for $8 \mathrm{~h}$. After completion of the reaction (monitored by TLC), the reaction mixture was concentrated under reduced pressure. The crude product was purified by column chromatography on silica gel (EtOAc-hexane) to afford the 7-benzyl-8tosyl-1,2,4,8-tetrahydro-3H-benzofuro[2,3-c]carbazol-3-one 3t, $223 \mathrm{mg}, 86 \%$ yield, off-white solid; $R_{f}=0.5$ (hexane: EtOAc $\left.=9: 1\right) ; \mathrm{mp} 164-166{ }^{\circ} \mathrm{C} ;{ }^{1} \mathrm{H} \mathrm{NMR}\left(500 \mathrm{MHz}, \mathrm{CDCl}_{3}\right) \delta 8.87$ $(\mathrm{d}, J=7.6 \mathrm{~Hz}, 1 \mathrm{H}), 8.16(\mathrm{~d}, J=8.1 \mathrm{~Hz}, 1 \mathrm{H}), 7.41-7.32(\mathrm{~m}, J=15.1,14.0,6.8 \mathrm{~Hz}, 2 \mathrm{H}), 7.28$ (s, 1H), $7.25(\mathrm{~s}, 1 \mathrm{H}), 7.22-7.14(\mathrm{~m}, 4 \mathrm{H}), 6.91(\mathrm{~d}, J=8.3 \mathrm{~Hz}, 2 \mathrm{H}), 6.79(\mathrm{~d}, J=8.2 \mathrm{~Hz}, 2 \mathrm{H})$, $4.82(\mathrm{~s}, 2 \mathrm{H}), 3.05(\mathrm{t}, J=6.3 \mathrm{~Hz}, 2 \mathrm{H}), 2.70-2.63(\mathrm{~m}, 2 \mathrm{H}), 2.28-2.21(\mathrm{~m}, 2 \mathrm{H}), 2.17(\mathrm{~s}, 3 \mathrm{H})$; ${ }^{13} \mathrm{C}$ NMR $\left(100 \mathrm{MHz}, \mathrm{CDCl}_{3}\right) \delta 193.2,172.1,154.0,144.3,142.4,141.6,138.4,133.6,131.1$, 130.7, 129.6, 128.5, 128.4 (2C), 127.3, 126.7, 126.2, 125.9,125.8, 119.2, 117.8, 114.0, 112.2, 40.0, 39.0, 24.6, 21.7, 21.5; IR (KBr): vmax $=1633,1373,1177,764,666 \mathrm{~cm}^{-1}$; HRMS (ESI): $m / z$ calcd for $\mathrm{C}_{32} \mathrm{H}_{26} \mathrm{NO}_{4} \mathrm{~S}(\mathrm{M}+\mathrm{H})^{+}: 520.1583$, found: 520.1579 .

\section{C) General Procedure: Synthesis of Annulated-Indoles}

Ethyl 2-methyl-1,4-diphenyl-5-(3-phenylprop-2-yn-1-yl)-1H-pyrrole-3-carboxylate (B):
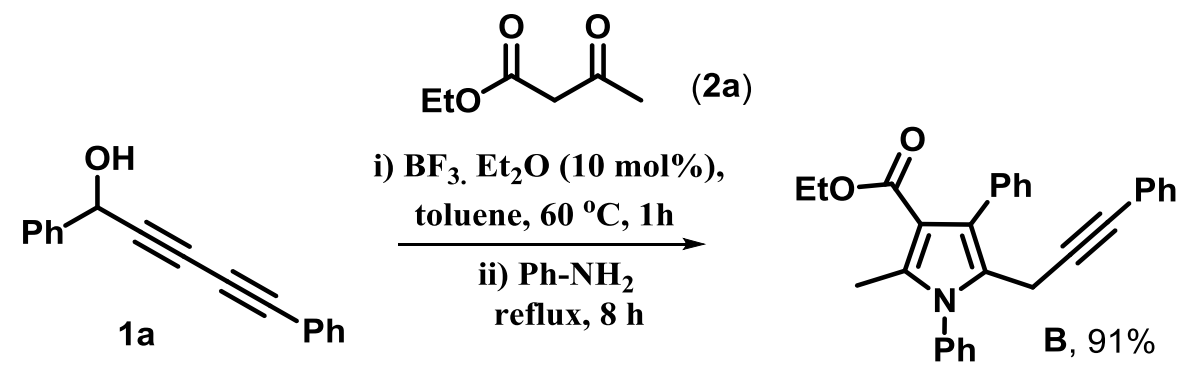

To a stirred solution of 1,5-diphenylpenta-2,4-diyn-1-ol $1 \mathbf{a}(116 \mathrm{mg}, 0.5 \mathrm{mmol})$ and ethyl 3oxobutanoate 2 (45 mg, $0.35 \mathrm{mmol})$ in $3 \mathrm{~mL}$ of toluene, was added $\mathrm{BF}_{3} \cdot \mathrm{Et}_{2} \mathrm{O}(7 \mathrm{mg}, 10$ 
$\mathrm{mol} \%$ ) and the mixture was stirred for $1 \mathrm{~h}$ at $60{ }^{\circ} \mathrm{C}$. Subsequently, aniline $(65 \mathrm{mg}, 0.6 \mathrm{mmol})$ was added and the mixture was heated to reflux for an additional $4 \mathrm{~h}$. After completion of the reaction (monitored by TLC), the reaction mixture was concentrated under reduced pressure and the crude product was purified by column chromatography on silica gel (EtOAchexanes) to afford the ethyl 2-methyl-1,4-diphenyl-5-(3-phenylprop-2-yn-1-yl)-1H-pyrrole-3carboxylate B, $190 \mathrm{mg}$, 91\% yield, off-white solid, $R_{f}=0.4$ (hexane: EtOAc $=9: 1$ ); mp 114$116{ }^{\circ} \mathrm{C} ;{ }^{1} \mathrm{H}$ NMR $\left(400 \mathrm{MHz}, \mathrm{CDCl}_{3}\right) \delta 7.50(\mathrm{~m}, 3 \mathrm{H}), 7.45-7.36(\mathrm{~m}, 6 \mathrm{H}), 7.31-7.25(\mathrm{~m}$, $6 \mathrm{H}), 4.10(\mathrm{q}, J=7.1 \mathrm{~Hz}, 2 \mathrm{H}), 3.37(\mathrm{~s}, 2 \mathrm{H}), 2.33(\mathrm{~s}, 3 \mathrm{H}), 1.03(\mathrm{t}, J=7.1 \mathrm{~Hz}, 3 \mathrm{H}),{ }^{13} \mathrm{C} \mathrm{NMR}$ $\left(100 \mathrm{MHz} \mathrm{CDCl}_{3}\right) \delta 165.8,137.2,136.8,135.7,131.4,130.5,129.3,128.9,128.8,128.2$, 127.7, 127.5, 126.3, 125.6, 123.6, 123.4, 111.3, 87.3, 80.7, 59.3, 16.4, 14.0, 12.6; IR (KBr): $v \max =1701,1258,1123,1082,762 \mathrm{~cm}^{-1}$; HRMS (ESI): $\mathrm{m} / z$ calcd for $\mathrm{C}_{29} \mathrm{H}_{26} \mathrm{NO}_{2}(\mathrm{M}+\mathrm{H})^{+}$: 420.1964, found: 420.1955 .
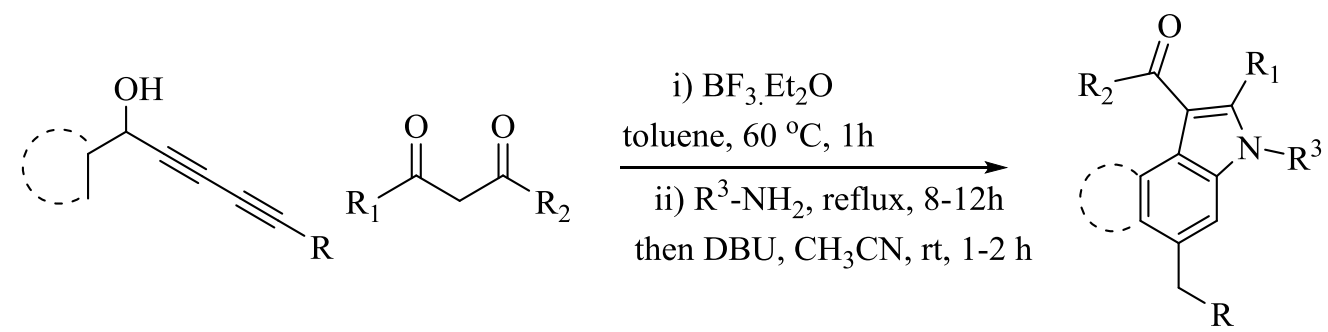

To a stirred solution of Diacetylinic propargylic alcohol 1 (0.5 mmol) and 1,3-dicarbonyl compounds $2(0.6 \mathrm{mmol})$ in $3 \mathrm{~mL}$ of Toluene in a reaction vial was added $\mathrm{BF}_{3} . \mathrm{Et}_{2} \mathrm{O}(10$ mol\%) The reaction mixture was stirred at $60{ }^{\circ} \mathrm{C}$, until completion of reaction. Followed by the addition of aniline $(0.6 \mathrm{mmol})$. The reaction mixture was heated to reflux for an additional 8-12 h until completion of reaction, then removed toluene add DBU (0.5 mmol) in $\mathrm{CH}_{3} \mathrm{CN}$ was added and stirred at $\mathrm{rt}$ for 1-2 hour. After completion of the reaction (monitored by TLC), the reaction mixture was concentrated under reduced pressure. The crude product was purified by column chromatography on silica gel (EtOAc-hexane) to afford the corresponding product.

\section{Ethyl 5-benzyl-2-methyl-3-phenyl-3H-benzo[e]indole-1-carboxylate (4a):}<smiles>CCOC(=O)c1c(C)n(-c2ccccc2)c2cc(Cc3ccccc3)c3ccccc3c12</smiles> 
Following the general procedure, to the solution of 1,5-diphenylpenta-2,4-diyn-1-ol 1a (116 mg, $0.5 \mathrm{mmol})$ and ethyl 3-oxobutanoate $\mathbf{2 a}(78 \mathrm{mg}, 0.6 \mathrm{mmol})$ in $3 \mathrm{~mL}$ of Toulene in a reaction vial was added $\mathrm{BF}_{3} \cdot \mathrm{Et}_{2} \mathrm{O}(7 \mathrm{mg}, 10 \mathrm{~mol} \%)$. The reaction mixture was stirred at $60{ }^{\circ} \mathrm{C}$ for $1 \mathrm{~h}$. Followed by the addition of aniline $(0.05 \mathrm{~mL}, 0.6 \mathrm{mmol})$. The reaction mixture was heated to reflux for an additional $8 \mathrm{~h}$ then removed toluene add DBU $(0.07 \mathrm{ml}, 0.5 \mathrm{mmol})$ in $\mathrm{CH}_{3} \mathrm{CN}$ was added and stirred at $\mathrm{rt}$ for 1 hour. After completion of the reaction (monitored by TLC), the reaction mixture was concentrated under reduced pressure. The crude product was purified by column chromatography on silica gel (EtOAc-hexane) to afford the Ethyl 5benzyl-2-methyl-3-phenyl-3H-benzo[e]indole-1-carboxylate 4a, $183 \mathrm{mg}, 87 \%$ yield, offwhite solid; $R_{f}=0.5$ (hexane: EtOAc $=9: 1$ ); mp $135-137{ }^{\circ} \mathrm{C} ;{ }^{1} \mathrm{H} \mathrm{NMR}\left(400 \mathrm{MHz}, \mathrm{CDCl}_{3}\right) \delta$ 9.17 (dd, $J=8.5,0.8 \mathrm{~Hz}, 1 \mathrm{H}), 7.94$ (d, $J=7.8 \mathrm{~Hz}, 1 \mathrm{H}), 7.60-7.51$ (m, 4H), $7.39-7.33$ (m, 3H), $7.20-7.15$ (m, 2H), $7.14-7.07$ (m, 3H), 7.02 (s, 1H), 4.53 (q, J = 7.1 Hz, 2H), 4.40 (s, 2H), $2.52(\mathrm{~s}, 3 \mathrm{H}), 1.49(\mathrm{t}, J=7.1 \mathrm{~Hz}, 3 \mathrm{H}) ;{ }^{13} \mathrm{C} \mathrm{NMR}\left(100 \mathrm{MHz}, \mathrm{CDCl}_{3}\right) \delta 167.2,140.9$, $140.9,136.7,134.3,132.0,129.8,129.5,128.9,128.5,128.4,128.3,128.2,126.3,125.8$, 125.3, 125.2, 123.9, 119.6, 113.5, 108.7, 60.4, 39.8, 14.5, 13.5; IR (KBr): $v_{\max }=1725,1251$, 1164, 761, $701 \mathrm{~cm}^{-1}$; HRMS (ESI): $\mathrm{m} / z$ calcd for $\mathrm{C}_{29} \mathrm{H}_{26} \mathrm{NO}_{2}(\mathrm{M}+\mathrm{H})^{+}$: 420.1964, found: 420.1975 .

Ethyl 5-benzyl-3-phenyl-2-(p-tolyl)-3H-benzo[e]indole-1-carboxylate (4b):

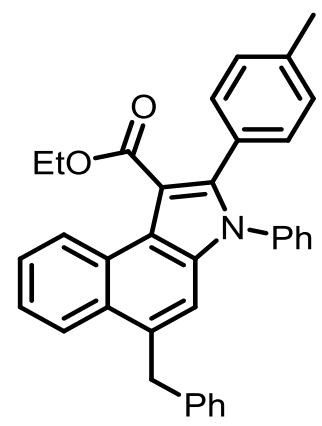

Following the general procedure, to the solution of 1,5-diphenylpenta-2,4-diyn-1-ol 1a (116 mg, $0.5 \mathrm{mmol})$ and ethyl 3-oxo-3-(p-tolyl) propanoate $2 \mathbf{d}(123 \mathrm{mg}, 0.6 \mathrm{mmol})$ in $3 \mathrm{~mL}$ of Toulene in a reaction vial was added $\mathrm{BF}_{3} \cdot \mathrm{Et}_{2} \mathrm{O}(7 \mathrm{mg}, 10 \mathrm{~mol} \%)$. The reaction mixture was stirred at 60 ${ }^{\circ} \mathrm{C}$, for $1 \mathrm{~h}$, followed by the addition of aniline $(0.05 \mathrm{~mL}, 0.6 \mathrm{mmol})$. The reaction mixture was heated to reflux for an additional $9 \mathrm{~h}$, then removed toluene the reaction mixture dissolved in $\mathrm{CH}_{3} \mathrm{CN}$ and added DBU $(0.07 \mathrm{ml}, 0.5 \mathrm{mmol})$ stirred at room temperature for 1 hour. After completion of the reaction (monitored by TLC), the reaction mixture was concentrated under reduced pressure. The crude product was purified by column 
chromatography on silica gel (EtOAc-hexane) to afford ethyl 5-benzyl-3-phenyl-2-(p-tolyl)3H-benzo[e]indole-1-carboxylate $4 \mathbf{b}, 194 \mathrm{mg}, 78 \%$ yield, off-white solid; $R_{f}=0.5$ (hexane: EtOAc = 8:2); mp $145-147{ }^{\circ} \mathrm{C} ;{ }^{1} \mathrm{H}$ NMR $\left(400 \mathrm{MHz} \mathrm{CDCl}_{3}\right) \delta 8.95(\mathrm{dd}, J=8.5,0.7 \mathrm{~Hz}, 1 \mathrm{H})$, $7.97(\mathrm{~d}, J=7.9 \mathrm{~Hz}, 1 \mathrm{H}), 7.57-7.53(\mathrm{~m}, 1 \mathrm{H}), 7.41-7.33(\mathrm{~m}, 5 \mathrm{H}), 7.21-7.18(\mathrm{~m}, 4 \mathrm{H}), 7.17$ $-7.11(\mathrm{~m}, 5 \mathrm{H}), 7.06(\mathrm{~d}, J=7.9 \mathrm{~Hz}, 2 \mathrm{H}), 4.44(\mathrm{~s}, 2 \mathrm{H}), 4.26(\mathrm{q}, J=7.1 \mathrm{~Hz}, 2 \mathrm{H}), 2.32(\mathrm{~s}, 3 \mathrm{H})$, $1.07(\mathrm{t}, J=7.1 \mathrm{~Hz}, 3 \mathrm{H}) ;{ }^{13} \mathrm{C} \mathrm{NMR}\left(125 \mathrm{MHz}, \mathrm{CDCl}_{3}\right) \delta 167.6,141.7,140.9,137.8,137.0$, $134.3,132.7,130.6,129.5,129.2$, 128.7, 128.6, 128.4, 128.4, 128.3, 128.3, 128.0, 125.9, $125.8,125.5,125.4,124.2,119.5,113.7,110.4,60.6,39.9,21.3,13.8$; IR $(\mathrm{KBr})$ : vmax $=$ 1709, 1499, 1184, 761, $703 \mathrm{~cm}^{-1}$; HRMS (ESI): $\mathrm{m} / z$ calcd for $\mathrm{C}_{35} \mathrm{H}_{30} \mathrm{NO}_{2}(\mathrm{M}+\mathrm{H})^{+}: 496.2277$, found: 496.2280 .

1-(5-benzyl-2-methyl-3-phenyl-3H-benzo[e]indol-1-yl) ethan-1-one (4c):

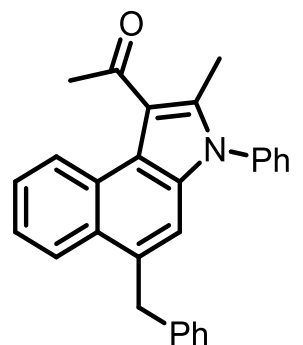

Following the general procedure, to the solution of 1,5-diphenylpenta-2,4-diyn-1-ol 1a (116 mg, $0.5 \mathrm{mmol})$ and pentane-2,4-dione $2 \mathbf{e}(60 \mathrm{mg}, 0.6 \mathrm{mmol})$ in $3 \mathrm{~mL}$ of Toulene in a reaction vial was added $\mathrm{BF}_{3} \cdot \mathrm{Et}_{2} \mathrm{O}(7 \mathrm{mg}, 10 \mathrm{~mol} \%)$. The reaction mixture was stirred at $60{ }^{\circ} \mathrm{C}$, for $1 \mathrm{~h}$, followed by the addition of aniline $(0.05 \mathrm{~mL}, 0.6 \mathrm{mmol})$. The reaction mixture was heated to reflux for an additional $9 \mathrm{~h}$, then removed toluene the reaction mixture dissolved in $\mathrm{CH}_{3} \mathrm{CN}$ and added DBU $(0.07 \mathrm{ml}, 0.5 \mathrm{mmol})$ stirred at room temperature for 1 hour. After completion of the reaction (monitored by TLC), the reaction mixture was concentrated under reduced pressure. The crude product was purified by column chromatography on silica gel (EtOAchexane) to afford the corresponding product 1-(5-benzyl-2-methyl-3-phenyl-3Hbenzo[e]indol-1-yl)ethan-1-one 4c, $168 \mathrm{mg}, 86 \%$ yield, off-white solid; $R_{f}=0.6$ (hexane: EtOAc $=8: 2) ; \mathrm{mp} 162-164{ }^{\circ} \mathrm{C} ;{ }^{1} \mathrm{H}$ NMR $\left(500 \mathrm{MHz}, \mathrm{CDCl}_{3}\right) \delta 8.46(\mathrm{~d}, J=8.3 \mathrm{~Hz}, 1 \mathrm{H}), 7.96$ $(\mathrm{d}, J=8.3 \mathrm{~Hz}, 1 \mathrm{H}), 7.58(\mathrm{t}, J=7.4 \mathrm{~Hz}, 2 \mathrm{H}), 7.57-7.50(\mathrm{~m}, 2 \mathrm{H}), 7.39-7.35(\mathrm{~m}, 3 \mathrm{H}), 7.19$ (t, $J=7.4 \mathrm{~Hz}, 2 \mathrm{H}), 7.14-7.09(\mathrm{~m}, 3 \mathrm{H}), 7.05(\mathrm{~s}, 1 \mathrm{H}), 4.41(\mathrm{~s}, 2 \mathrm{H}), 2.76(\mathrm{~s}, 3 \mathrm{H}), 2.45(\mathrm{~s}, 3 \mathrm{H}),{ }^{13} \mathrm{C}$ NMR $\left(100 \mathrm{MHz}, \mathrm{CDCl}_{3}\right) \delta 200.0,140.9,137.5,136.6,134.2,132.3,129.8,129.4,128.9$, 128.4, 128.3 (2C), 128.0, 125.9, 125.7, 125.6, 125.5, 123.9, 119.9, 119.2, 113.5, 39.8, 32.3, 13.0; IR (KBr): vmax $=1665,1400,761,707 \mathrm{~cm}^{-1}$; HRMS (ESI): $\mathrm{m} / z$ calcd for $\mathrm{C}_{28} \mathrm{H}_{24} \mathrm{NO}$ $(\mathrm{M}+\mathrm{H})^{+}:$390.1858, found: 390.1864 . 


\section{5-Benzyl-7-phenyl-7,8,9,10-tetrahydro-11H-benzo[c]carbazol-11-one (4d):}<smiles>O=C1CCCc2c1c1c3ccccc3c(Cc3ccccc3)cc1n2-c1ccccc1</smiles>

Following the general procedure, to the solution of 1,5-diphenylpenta-2,4-diyn-1-ol 1a (116 mg, $0.5 \mathrm{mmol})$ and cyclohexane-1,3-dione $2 \mathbf{f}(67 \mathrm{mg}, 0.6 \mathrm{mmol})$ in $3 \mathrm{~mL}$ of Toulene in a reaction vial was added $\mathrm{BF}_{3} \cdot \mathrm{Et}_{2} \mathrm{O}(7 \mathrm{mg}, 10 \mathrm{~mol} \%)$. The reaction mixture was stirred at $60{ }^{\circ} \mathrm{C}$, for $1 \mathrm{~h}$, followed by the addition of aniline $(0.05 \mathrm{~mL}, 0.6 \mathrm{mmol})$. The reaction mixture was heated to reflux for an additional $10 \mathrm{~h}$, then removed toluene the reaction mixture dissolved in $\mathrm{CH}_{3} \mathrm{CN}$ and added DBU $(0.07 \mathrm{ml}, 0.5 \mathrm{mmol})$ stirred at room temperature for 1 hour. After completion of the reaction (monitored by TLC), the reaction mixture was concentrated under reduced pressure. The crude product was purified by column chromatography on silica gel (EtOAchexane) to afford the corresponding product 5-benzyl-7-phenyl-7,8,9,10-tetrahydro- $11 \mathrm{H}$ benzo[c]carbazol-11-one 4d, $165 \mathrm{mg}, 82 \%$ yield, Brown solid; $R_{f}=0.8$ (hexane: EtOAc $=8: 2$ ); mp 158-160 ${ }^{\circ} \mathrm{C} ;{ }^{1} \mathrm{H}$ NMR $\left(400 \mathrm{MHz}, \mathrm{CDCl}_{3}\right) \delta 10.13$ (d, $\left.J=8.4 \mathrm{~Hz}, 1 \mathrm{H}\right), 7.96$ (d, $J=8.4 \mathrm{~Hz}$, 1H), $7.66-7.53(\mathrm{~m}, 4 \mathrm{H}), 7.46-7.39(\mathrm{~m}, 3 \mathrm{H}), 7.19(\mathrm{t}, J=7.2 \mathrm{~Hz}, 2 \mathrm{H}), 7.14-7.07(\mathrm{~m}, 4 \mathrm{H})$, $4.43(\mathrm{~s}, 2 \mathrm{H}), 2.87(\mathrm{t}, J=6.2 \mathrm{~Hz}, 2 \mathrm{H}), 2.80-2.72(\mathrm{~m}, 2 \mathrm{H}), 2.26-2.19(\mathrm{~m}, 2 \mathrm{H}) ;{ }^{13} \mathrm{C} \mathrm{NMR}$ $\left(125 \mathrm{MHz}, \mathrm{CDCl}_{3}\right) \delta 193.7,150.2,140.8,136.1,134.9,133.4,129.9,129.6,129.2,129.1$, 129.0, 128.3 (2C), 127.8, 125.9, 125.9, 124.7, 124.6, 119.9, 116.2, 113.0, 39.8, 39.7, 23.9, 23.3; IR (KBr): vmax $=1652,1506,1451,760,708 \mathrm{~cm}^{-1}$; HRMS (ESI): $\mathrm{m} / \mathrm{z}$ calcd for $\mathrm{C}_{29} \mathrm{H}_{24} \mathrm{NO}(\mathrm{M}+\mathrm{H})^{+}: 402.1858$, found: 402.1869 .

Ethyl 3,5-dibenzyl-2-methyl-3H-benzo[e]indole-1-carboxylate (4e): 


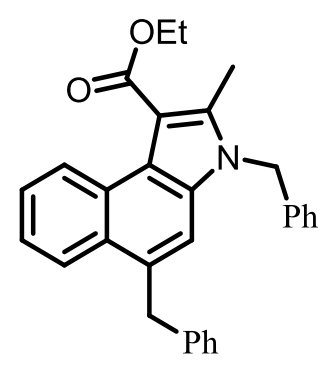

Following the general procedure, to the solution of 1,5-diphenylpenta-2,4-diyn-1-ol 1a (116 mg, $0.5 \mathrm{mmol}$ ) and ethyl 3-oxobutanoate $\mathbf{2 a}(78 \mathrm{mg}, 0.6 \mathrm{mmol})$ in $3 \mathrm{~mL}$ of Toulene in a reaction vial was added $\mathrm{BF}_{3} \cdot \mathrm{Et}_{2} \mathrm{O}(7 \mathrm{mg}, 10 \mathrm{~mol} \%)$. The reaction mixture was stirred at $60{ }^{\circ} \mathrm{C}$, for $1 \mathrm{~h}$, followed by the addition of Benzylamine $(0.06 \mathrm{~mL}, 0.6 \mathrm{mmol})$. The reaction mixture was heated to reflux for an additional $8 \mathrm{~h}$, then removed toluene the reaction mixture dissolved in $\mathrm{CH}_{3} \mathrm{CN}$ and added $\mathrm{DBU}(0.07 \mathrm{ml}, 0.5 \mathrm{mmol})$ stirred at room temperature for 1 hour. After completion of the reaction (monitored by TLC), the reaction mixture was concentrated under reduced pressure. The crude product was purified by column chromatography on silica gel (EtOAc-hexane) to afford the corresponding product Ethyl 3,5-dibenzyl-2-methyl-3Hbenzo[e]indole-1-carboxylate 4e, $182 \mathrm{mg}, 84 \%$ yield, off-white solid; $R_{f}=0.7$ (hexane: EtOAc = 8:2); mp 125-127 ${ }^{\circ} \mathrm{C} ;{ }^{1} \mathrm{H}$ NMR (400 MHz, $\left.\mathrm{CDCl}_{3}\right) \delta 9.13(\mathrm{dd}, J=8.6,0.8 \mathrm{~Hz}, 1 \mathrm{H}), 7.99(\mathrm{~d}$, $J=7.7 \mathrm{~Hz}, 1 \mathrm{H}), 7.56-7.49$ (m, 1H), $7.41-7.35$ (m, 1H), 7.28 - 7.26 (m, 1H), 7.26 - 7.24 $(\mathrm{m}, 2 \mathrm{H}), 7.23-7.14(\mathrm{~m}, 4 \mathrm{H}), 7.13-7.08(\mathrm{~m}, 2 \mathrm{H}), 6.95(\mathrm{dd}, J=7.4,2.0 \mathrm{~Hz}, 2 \mathrm{H}), 5.40(\mathrm{~s}$, $2 \mathrm{H}), 4.51(\mathrm{q}, J=7.1 \mathrm{~Hz}, 2 \mathrm{H}), 4.44(\mathrm{~s}, 2 \mathrm{H}), 2.66(\mathrm{~s}, 3 \mathrm{H}), 1.48(\mathrm{t}, J=7.1 \mathrm{~Hz}, 3 \mathrm{H}) ;{ }^{13} \mathrm{C}$ NMR $\left(125 \mathrm{MHz}, \mathrm{CDCl}_{3}\right) \delta 167.2,140.7,140.3,136.5,133.1,132.3,129.3,128.9,128.6,128.5$, 128.3, 127.6, 126.2, 126.0, 125.9, 125.3, 124.9, 123.8, 119.7, 112.5, 108.4, 60.3, 46.8, 39.8, 14.5, 12.4; IR (KBr): $v \max =1698,1413,1115,760,710 \mathrm{~cm}^{-1}$; HRMS (ESI): $\mathrm{m} / z$ calcd for $\mathrm{C}_{30} \mathrm{H}_{28} \mathrm{NO}_{2}(\mathrm{M}+\mathrm{H})^{+}:$434.2120, found: 434.2124.

Ethyl 5-benzyl-3-(4-methoxybenzyl)-2-methyl-3H-benzo[e]indole-1-carboxylate (4f):

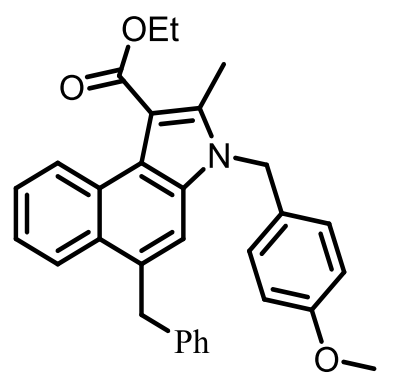

Following the general procedure, to the solution of 1,5-diphenylpenta-2,4-diyn-1-ol 1a (116 mg, $0.5 \mathrm{mmol}$ ) and ethyl 3-oxobutanoate $\mathbf{2 a}(78 \mathrm{mg}, 0.6 \mathrm{mmol})$ in $3 \mathrm{~mL}$ of Toulene in a reaction vial was added $\mathrm{BF}_{3} \cdot \mathrm{Et}_{2} \mathrm{O}(7 \mathrm{mg}, 10 \mathrm{~mol} \%)$. The reaction mixture was stirred at $60{ }^{\circ} \mathrm{C}$, for $1 \mathrm{~h}$, 
followed by the addition of 4-methoxy benzylamine $(0.06 \mathrm{~mL}, 0.6 \mathrm{mmol})$. The reaction mixture was heated to reflux for an additional $9 \mathrm{~h}$, then removed toluene the reaction mixture dissolved in $\mathrm{CH}_{3} \mathrm{CN}$ and added DBU $(0.07 \mathrm{ml}, 0.5 \mathrm{mmol})$ stirred at room temperature for 1 hour. After completion of the reaction (monitored by TLC), the reaction mixture was concentrated under reduced pressure. The crude product was purified by column chromatography on silica gel (EtOAc-hexane) to afford the corresponding product ethyl 5benzyl-3-(4-methoxybenzyl)-2-methyl-3H-benzo[e]indole-1-carboxylate 4f, $188 \mathrm{mg}, 81 \%$ yield, off-white solid; $R_{f}=0.6$ (hexane: EtOAc $=8: 2$ ); mp $132-134{ }^{\circ} \mathrm{C} ;{ }^{1} \mathrm{H}$ NMR $(400 \mathrm{MHz}$, $\left.\mathrm{CDCl}_{3}\right) \delta 9.12(\mathrm{dd}, J=8.5,0.8 \mathrm{~Hz}, 1 \mathrm{H}), 7.99(\mathrm{~d}, J=7.8 \mathrm{~Hz}, 1 \mathrm{H}), 7.52(\mathrm{~m}, 1 \mathrm{H}), 7.38(\mathrm{~m}, 1 \mathrm{H})$, $7.24-7.10(\mathrm{~m}, 6 \mathrm{H}), 6.88(\mathrm{~d}, J=8.8 \mathrm{~Hz}, 2 \mathrm{H}), 6.81-6.76(\mathrm{~m}, 2 \mathrm{H}), 5.33(\mathrm{~s}, 2 \mathrm{H}), 4.50$ (q, $J=$ $7.1 \mathrm{~Hz}, 2 \mathrm{H}), 4.45$ (s, 2H), $3.76(\mathrm{~s}, 3 \mathrm{H}), 2.66(\mathrm{~s}, 3 \mathrm{H}), 1.47(\mathrm{t}, J=7.1 \mathrm{~Hz}, 3 \mathrm{H}) ;{ }^{13} \mathrm{C} \mathrm{NMR}(125$ $\left.\mathrm{MHz}, \mathrm{CDCl}_{3}\right) \delta 167.2,159.0,140.8,140.3,133.0,132.2,129.2,128.7$ (2C), 128.5, 128.4, 127.3, 126.2, 125.9, 125.3, 124.9, 123.8, 119.7, 114.3, 112.5, 108.3, 60.3, 55.3, 46.3, 39.8, 14.5, 12.4; IR (KBr): vmax $=1699,1517,1253,1115,762 \mathrm{~cm}^{-1}$; HRMS (ESI): $\mathrm{m} / z$ calcd for $\mathrm{C}_{31} \mathrm{H}_{30} \mathrm{NO}_{3}(\mathrm{M}+\mathrm{H})^{+}:$464.2226, found: 464.2232.

\section{Ethyl5-benzyl-7-ethyl-2-methyl-3-phenyl-3,7-dihydroindolo[5,4-b]carbazole-1- carboxylate $(4 \mathrm{~g})$ :}

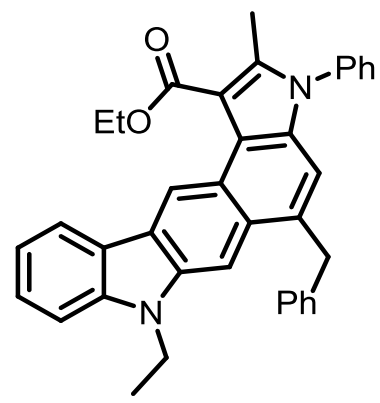

Following the general procedure, to the solution of 1-(9-ethyl-9H-carbazol-3-yl)-5-phenylpenta2,4-diyn-1-ol 1e (174 mg, $0.5 \mathrm{mmol})$ and ethyl 3-oxobutanoate $\mathbf{2 a}(78 \mathrm{mg}, 0.6 \mathrm{mmol})$ in $3 \mathrm{~mL}$ of Toulene in a reaction vial was added $\mathrm{BF}_{3} . \mathrm{Et}_{2} \mathrm{O}(7 \mathrm{mg}, 10 \mathrm{~mol} \%)$. The reaction mixture was stirred at $60{ }^{\circ} \mathrm{C}$, for $1 \mathrm{~h}$, followed by the addition of aniline $(0.05 \mathrm{~mL}, 0.6 \mathrm{mmol})$. The reaction mixture was heated to reflux for an additional $8 \mathrm{~h}$, then removed toluene the reaction mixture dissolved in $\mathrm{CH}_{3} \mathrm{CN}$ and added $\mathrm{DBU}(0.07 \mathrm{ml}, 0.5 \mathrm{mmol})$ stirred at room temperature for 2 hours. After completion of the reaction (monitored by TLC), the reaction mixture was concentrated under reduced pressure. The crude product was purified by column chromatography on silica gel (EtOAc-hexane) to afford the ethyl 5-benzyl-7-ethyl-2-methyl3-phenyl-3,7-dihydroindolo[5,4-b]carbazole-1-carboxylate 4g, 212 mg, 79\% yield, brown 
solid; $R_{f}=0.5$ (hexane: EtOAc $\left.=8: 2\right) ; \mathrm{mp} 110-112{ }^{\circ} \mathrm{C} ;{ }^{1} \mathrm{H} \mathrm{NMR}\left(300 \mathrm{MHz}, \mathrm{CDCl}_{3}\right) \delta 9.14$ $(\mathrm{d}, J=9.2 \mathrm{~Hz}, 1 \mathrm{H}), 8.17(\mathrm{~d}, J=8.2 \mathrm{~Hz}, 1 \mathrm{H}), 7.71(\mathrm{~d}, J=9.2 \mathrm{~Hz}, 1 \mathrm{H}), 7.57-7.48(\mathrm{~m}, 4 \mathrm{H})$, $7.42-7.37(\mathrm{~m}, 1 \mathrm{H}), 7.27(\mathrm{~d}, J=1.8 \mathrm{H}, 1 \mathrm{H}), 7.18-7.10(\mathrm{~m}, 2 \mathrm{H}), 7.09-7.01(\mathrm{~m}, 4 \mathrm{H}), 6.88$ $(\mathrm{d}, J=6.4 \mathrm{~Hz}, 2 \mathrm{H}), 4.90(\mathrm{~s}, 2 \mathrm{H}), 4.60-4.49(\mathrm{~m}, 4 \mathrm{H}), 2.52$ (s, 3H), 1.52 (d, J=4.4 Hz, 3H), $1.49(\mathrm{t}, J=5.9 \mathrm{~Hz}, 3 \mathrm{H}) ;{ }^{13} \mathrm{C} \mathrm{NMR}\left(100 \mathrm{MHz}, \mathrm{CDCl}_{3}\right) \delta 167.4,143.4,141.5,138.7,138.2$, $136.8,134.0,132.0,129.6,128.8,128.6,128.3$, 128.0, 127.0, 125.7, 125.4, 124.7, 124.4, $123.4,123.3,120.5,118.1,115.6,114.6,108.7,108.5,108.2,60.3,41.7,37.7,14.6,14.2$, 13.6; IR (KBr): vmax $=1699,1514,1412,1172,762 \mathrm{~cm}^{-1}$; HRMS (ESI): $\mathrm{m} / z$ calcd for $\mathrm{C}_{37} \mathrm{H}_{33} \mathrm{~N}_{2} \mathrm{O}_{2}(\mathrm{M}+\mathrm{H})^{+}:$537.2542, found: 537.2528.

Ethyl 4-benzyl-7-methyl-6-phenyl-6H-thieno[2,3-e] indole-8-carboxylate (4h):

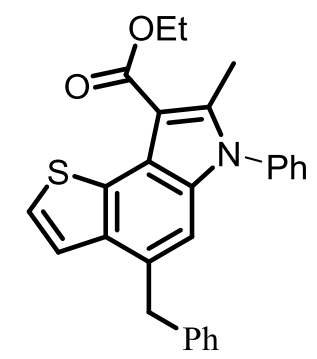

Following the general procedure, to the solution of 5-phenyl-1-(thiophen-3-yl) penta-2,4-diyn-1-ol 1f $(119 \mathrm{mg}, 0.5 \mathrm{mmol})$ and ethyl 3-oxobutanoate $\mathbf{2 a}(78 \mathrm{mg}, 0.6 \mathrm{mmol})$ in $3 \mathrm{~mL}$ of Toulene in a reaction vial was added $\mathrm{BF}_{3} \cdot \mathrm{Et}_{2} \mathrm{O}(7 \mathrm{mg}, 10 \mathrm{~mol} \%)$. The reaction mixture was stirred at 60 ${ }^{\circ} \mathrm{C}$, for $1 \mathrm{~h}$, followed by the addition of aniline $(0.05 \mathrm{~mL}, 0.6 \mathrm{mmol})$. The reaction mixture was heated to reflux for an additional $10 \mathrm{~h}$, then removed toluene the reaction mixture dissolved in $\mathrm{CH}_{3} \mathrm{CN}$ and added $\mathrm{DBU}(0.07 \mathrm{ml}, 0.5 \mathrm{mmol})$ stirred at room temperature for 1 hour. After completion of the reaction (monitored by TLC), the reaction mixture was concentrated under reduced pressure. The crude product was purified by column chromatography on silica gel (EtOAc-hexane) to afford the ethyl 4-benzyl-7-methyl-6phenyl-6H-thieno[2,3-e]indole-8-carboxylate $4 \mathbf{h}, 193 \mathrm{mg}, 91 \%$ yield, brown solid; $R_{f}=0.6$ (hexane: EtOAc = 9:1); $\mathrm{mp} 110-112{ }^{\circ} \mathrm{C} ;{ }^{1} \mathrm{H}$ NMR (500 MHz, $\left.\mathrm{CDCl}_{3}\right) \delta 7.60-7.52(\mathrm{~m}, 3 \mathrm{H})$, $7.38-7.33(\mathrm{~m}, 4 \mathrm{H}), 7.21-7.17(\mathrm{~m}, 2 \mathrm{H}), 7.14-7.08(\mathrm{~m}, 3 \mathrm{H}), 6.85(\mathrm{~s}, 1 \mathrm{H}), 4.56$ (q, J = 7.1 $\mathrm{Hz}, 2 \mathrm{H}), 4.30$ (s, 2H), 2.60 (s, 3H), 1.52 (t, $J=7.1 \mathrm{~Hz}, 3 \mathrm{H}) ;{ }^{13} \mathrm{C} \mathrm{NMR}\left(100 \mathrm{MHz}, \mathrm{CDCl}_{3}\right) \delta$ $164.73,142.24,139.85,135.71,134.71,133.83,131.32,129.04,128.80,127.99$, 127.41, $127.35,127.28,124.90,123.66,121.18,118.48,108.66,104.26,58.88,39.38,13.80,12.40$; IR $(\mathrm{KBr}): v \max =1701,1194,759,709 \mathrm{~cm}^{-1}$; HRMS (ESI): $\mathrm{m} / z$ calcd for $\mathrm{C}_{27} \mathrm{H}_{24} \mathrm{NO}_{2} \mathrm{~S}$ $(\mathrm{M}+\mathrm{H})^{+}:$426.1521, found: 426.1528 . 

carboxylate (4i):

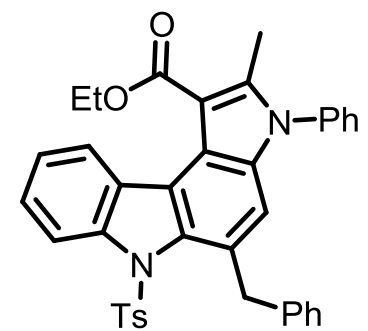

Following the general procedure, to the solution of 5-phenyl-1-(1-tosyl-1H-indol-3-yl) penta-2,4diyn-1-ol 1i (212 mg, $0.5 \mathrm{mmol})$ and ethyl 3-oxobutanoate $\mathbf{2 a}(78 \mathrm{mg}, 0.6 \mathrm{mmol})$ in $3 \mathrm{~mL}$ of Toulene in a reaction vial was added $\mathrm{BF}_{3} . \mathrm{Et}_{2} \mathrm{O}(7 \mathrm{mg}, 10 \mathrm{~mol} \%)$. The reaction mixture was stirred at $60{ }^{\circ} \mathrm{C}$, for $1 \mathrm{~h}$, followed by the addition of aniline $(0.05 \mathrm{~mL}, 0.6 \mathrm{mmol})$. The reaction mixture was heated to reflux for an additional $9 \mathrm{~h}$, then removed toluene the reaction mixture dissolved in $\mathrm{CH}_{3} \mathrm{CN}$ and added $\mathrm{DBU}(0.07 \mathrm{ml}, 0.5 \mathrm{mmol})$ stirred at room temperature for 1 hour. After completion of the reaction (monitored by TLC), the reaction mixture was concentrated under reduced pressure. The crude product was purified by column chromatography on silica gel (EtOAc-hexane) to afford the ethyl 5-benzyl-2-methyl-3phenyl-6-tosyl-3,6-dihydropyrrolo[2,3-c]carbazole-1-carboxylate 4i, $255 \mathrm{mg}, 83 \%$ yield, offwhite solid; $R_{f}=0.6$ (hexane: EtOAc $=9: 1$ ); mp 152-154 ${ }^{\circ} \mathrm{C} ;{ }^{1} \mathrm{H}$ NMR $\left(500 \mathrm{MHz}, \mathrm{CDCl}_{3}\right) \delta$ $8.17(\mathrm{~d}, J=8.1 \mathrm{~Hz}, 1 \mathrm{H}), 7.50(\mathrm{t}, J=7.1 \mathrm{~Hz}, 3 \mathrm{H}), 7.44(\mathrm{~d}, J=7.7 \mathrm{~Hz}, 1 \mathrm{H}), 7.34-7.30(\mathrm{~m}$, 1H), $7.25-7.21(\mathrm{~m}, 3 \mathrm{H}), 7.14(\mathrm{t}, J=7.2 \mathrm{~Hz}, 2 \mathrm{H}), 7.10-7.05(\mathrm{~m}, 3 \mathrm{H}), 7.02(\mathrm{~d}, J=8.3 \mathrm{~Hz}$, 2H), $6.85(\mathrm{~d}, J=8.2 \mathrm{~Hz}, 2 \mathrm{H}), 6.74(\mathrm{~s}, 1 \mathrm{H}), 4.74(\mathrm{~s}, 2 \mathrm{H}), 4.28$ (q, J = $7.1 \mathrm{~Hz}, 2 \mathrm{H}), 2.50$ (s, $3 \mathrm{H}), 2.17(\mathrm{~s}, 3 \mathrm{H}), 0.95(\mathrm{t}, J=7.2 \mathrm{~Hz}, 3 \mathrm{H}) ;{ }^{13} \mathrm{C} \mathrm{NMR}\left(100 \mathrm{MHz}, \mathrm{CDCl}_{3}\right) \delta 166.5,144.2$, $143.7,142.2$, 141.8, 137.2, 137.1, 136.3, 131.5, 131.4, 130.5, 129.7, 129.2, 128.9, 128.4, 128.1 (2C), 128.0, 127.5, 125.7, 124.9, 124.2, 123.7, 119.6, 117.3, 112.2, 106.9, 60.0, 39.6, 21.4, 13.8, 12.9; IR (KBr): $v \max =1214,1172,757,666 \mathrm{~cm}^{-1}$; HRMS (ESI): $\mathrm{m} / z$ calcd for $\mathrm{C}_{38} \mathrm{H}_{33} \mathrm{O}_{4} \mathrm{~N}_{2} \mathrm{~S}(\mathrm{M}+\mathrm{H})^{+}:$613.2167, found: 613.2155 .

\section{4) Gram scale reaction and derivatization:}

1-(5-Benzyl-7-methoxy-2-methylnaphtho[2,1-b] furan-1-yl) ethan-1-one (3m):<smiles>COc1ccc2c(c1)c(Cc1ccccc1)cc1oc(C)c(C(C)=O)c12</smiles> 
Following the general procedure, to the solution of 1-(4-methoxyphenyl)-5-phenylpenta-2,4-diyn1-ol $1 \mathbf{b}(1 \mathrm{~g}, 3.8 \mathrm{mmol})$ and pentane-2,4-dione $2 \mathbf{e}(286 \mathrm{mg}, 0.35 \mathrm{mmol})$ in $3 \mathrm{~mL}$ of acetonitrile in a reaction vial was added $\mathrm{BF}_{3} \cdot \mathrm{Et}_{2} \mathrm{O}(0.1 \mathrm{~mL}, 10 \mathrm{~mol} \%)$ at $0{ }^{\circ} \mathrm{C}$ and moved to room temperature and stirred for $30 \mathrm{~min}$. Then, $\mathrm{K}_{2} \mathrm{CO}_{3}(1.31 \mathrm{~g}, 1.25 \mathrm{mmol})$ was added and stirred at $80{ }^{\circ} \mathrm{C}$ (oil bath temperature) for $8 \mathrm{~h}$. After completion of the reaction (monitored by TLC), the reaction mixture was concentrated under reduced pressure. The crude product was purified by column chromatography on silica gel (EtOAc-hexane) to afford the corresponding product 1-(5-benzyl-7-methoxy-2-methylnaphtho[2,1-b]furan-1-yl)ethan-1one 3m, $1.06 \mathrm{~g}, 81 \%$ yield.

(E)-1-(5-Benzyl-7-methoxy-2-methylnaphtho[2,1-b]furan-1-yl)ethan-1-one oxime (5):
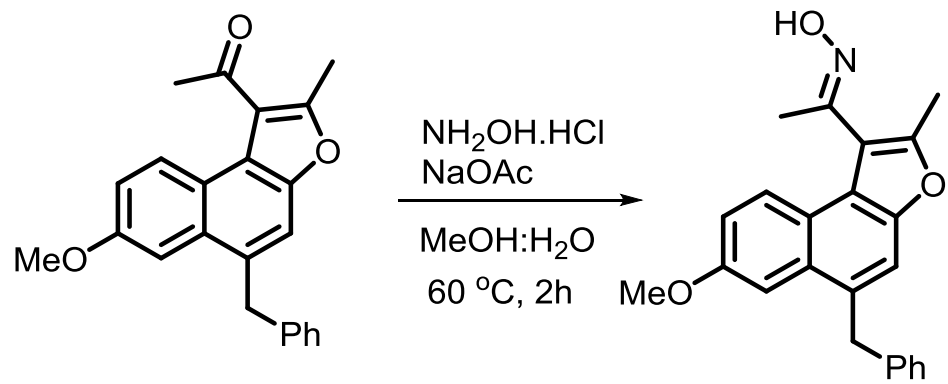

To the solution of 1-(5-benzyl-7-methoxy-2-methylnaphtho[2,1-b] furan-1-yl) ethan-1-one 3m (100 mg, $0.29 \mathrm{mmol}$ ) and Hydroxylamine hydrochloride (40 mg, $0.34 \mathrm{mmol})$, Sodium acetate (36 mg, 0.43) in $10 \mathrm{~mL}$ of $\mathrm{MeOH}: \mathrm{H}_{2} \mathrm{O}(1: 1)$ at $0{ }^{\circ} \mathrm{C}$. The reaction mixture was stirred at $60{ }^{\circ} \mathrm{C}$, for 2h. After completion of the reaction (monitored by TLC), the reaction mixture was concentrated under reduced pressure. The crude product was purified by column chromatography on silica gel (EtOAc-hexane) to afford the $(E)$-1-(5-benzyl-7-methoxy-2methylnaphtho[2,1-b] furan-1-yl) ethan-1-one oxime 5, semi-solid, $96 \mathrm{mg}, 92 \%$ yield, $R_{f}=$ 0.5 (hexane: EtOAc $=4: 6) ;{ }^{1} \mathrm{H}$ NMR $\left(400 \mathrm{MHz} \mathrm{CDCl}_{3}\right) \delta 7.94(\mathrm{~d}, J=9.1 \mathrm{~Hz}, 1 \mathrm{H}), 7.40(\mathrm{~s}$, 1H), $7.36(\mathrm{~d}, J=2.5 \mathrm{~Hz}, 1 \mathrm{H}), 7.30-7.27(\mathrm{~m}, 2 \mathrm{H}), 7.24-7.16(\mathrm{~m}, 4 \mathrm{H}), 4.44(\mathrm{~s}, 2 \mathrm{H}), 3.80(\mathrm{~s}$, $3 \mathrm{H}), 2.50(\mathrm{~s}, 3 \mathrm{H}), 2.34(\mathrm{~s}, 3 \mathrm{H}) ;{ }^{13} \mathrm{C} \mathrm{NMR}\left(100 \mathrm{MHz}, \mathrm{CDCl}_{3}\right) \delta 156.4,152.6,152.2,150.3$, $140.3,132.7,130.7,128.8,128.6,126.2,125.4,122.5,120.7,117.4,114.9,113.8,105.3$, 55.2, 40.0, 16.5, 12.6; IR (KBr): vmax $=3818,3583,2924,1604,1240,710 \mathrm{~cm}^{-1}$; HRMS (ESI): $m / z$ calcd for $\mathrm{C}_{23} \mathrm{H}_{22} \mathrm{NO}_{3}(\mathrm{M}+\mathrm{H})^{+}: 360.1600$, found: 360.1595 .

\section{5) Crystal Structure determination of 5 :}

\section{X-ray Crystallography:}


X-ray data for the compound was collected at room temperature on a Bruker D8 QUEST instrument with an $\mathrm{I} \mu \mathrm{S}$ Mo microsource $(\lambda=0.7107 \mathrm{~A})$ and a PHOTON-100 detector. The raw data frames were reduced and corrected for absorption effects using the Bruker Apex 3 software suite programs ${ }^{3}$. The structure was solved using intrinsic phasing method ${ }^{2}$ and further refined with the SHELXL ${ }^{4}$ program and expanded using Fourier techniques. Anisotropic displacement parameters were included for all non-hydrogen atoms. All C bound $\mathrm{H}$ atoms were positioned geometrically and treated as riding on their parent $\mathrm{C}$ atoms $[\mathrm{C}-\mathrm{H}=$ 0.93-0.97 $\AA$, and $\operatorname{Uiso}(\mathrm{H})=1.5 \mathrm{Ueq}(\mathrm{C})$ for methyl $\mathrm{H}$ or $1.2 \mathrm{Ueq}(\mathrm{C})$ for other $\mathrm{H}$ atoms]. The $\mathrm{O}$ bound $\mathrm{H}$ atoms were located in the difference Fourier map and the positional parameters of $\mathrm{H}$ atoms were refined. The carbon and oxygen atoms of methoxy group are disordered over two sites [3]. The site occupancy factor for major component of the disordered atoms (C7/O1) is $0.772(6)$ and the minor component of the disordered atoms (C7D/O1D) is $0.228(6)$.

Crystal data for compound 5: $\mathrm{C}_{29} \mathrm{H}_{21} \mathrm{~N}_{1} \mathrm{O}_{3}, M=359.41$, Monoclinic, space group $C 2 / c$ (No.15), $a=20.6964(6) \AA, b=7.2666(2) \AA, c=25.2715(8) \AA, \alpha=90^{\circ}, \beta=98.8302(14)^{\circ}, \gamma=$ $90^{\circ}, V=3755.60(19) \AA^{3}, Z=8, D_{\mathrm{c}}=1.271 \mathrm{~g} / \mathrm{cm}^{3}, F_{000}=1520$, Bruker D8 QUEST PHOTON-100, Mo-K $\alpha$ radiation, $\lambda=0.71073 \AA, T=293(2) \mathrm{K}, 2 \theta_{\max }=55^{\circ}, \mu=0.084 \mathrm{~mm}^{-1}$, 27397 reflections collected, 4316 unique $\left(\mathrm{R}_{\text {int }}=0.0430\right), 271$ parameters, $R 1=0.0511, w R 2=$ $0.1082, R$ indices based on 3069 reflections with $\mathrm{I}>2 \sigma(\mathrm{I})$ (refinement on $F^{2}$ ), Final GooF $=$ 1.035, largest difference hole and peak $=-0.151$ and 0.178 e. $\AA^{-3}$. CCDC 2086647 deposition numbers contains the supplementary crystallographic data for this paper which can be obtained free of charge at https://www.ccdc.cam.ac.uk/structures/ 


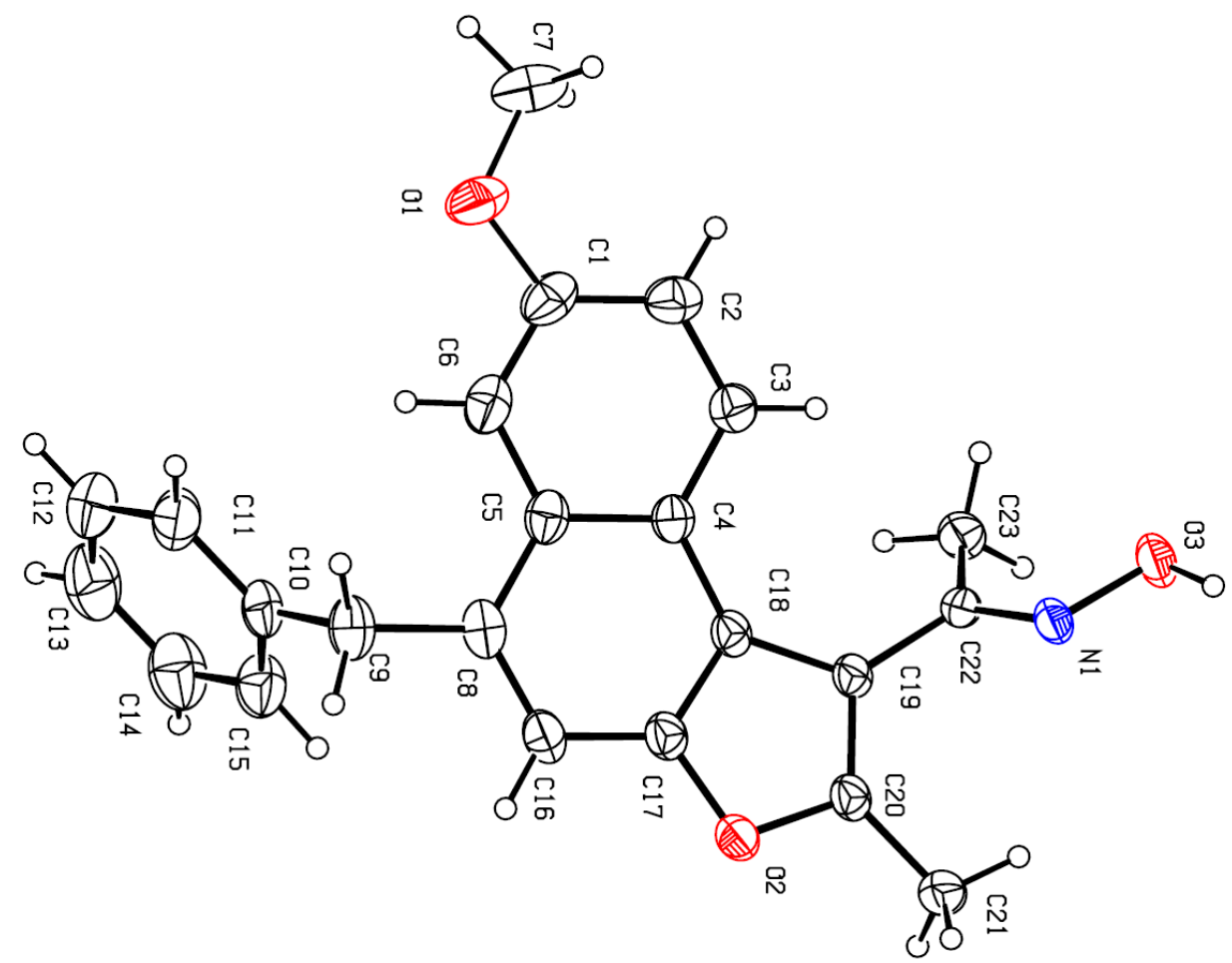

Figure caption: ORTEP diagram compound 5 with the atom-numbering. Displacement ellipsoids are drawn at the $35 \%$ probability level and $\mathrm{H}$ atoms are shown as small spheres of arbitrary radius. Minor component of the disordered methoxy group carbon and oxygen atoms are omitted for clarity.

\section{6) References:}

1) (a) Chen, J.; Fan, G.; Liu, Y. Stereoselective synthesis of enynones via base-catalyzed isomerization of 1,5-disubstituted-2,4-pentadiynyl silyl ethers or their alcohol derivatives. Org. Biomol. Chem. 2010, 8, 4806-4810. (b) Chinta, B. S.; Baire, B. A systematic study on the Cadiot-Chodkiewicz cross coupling reaction for the selective and efficient synthesis of hetero-diynes. RSC $A d v$. 2016, 6, 54449-54455. (c) Raji Reddy, C.; Rani Valleti, R.; Sathish, P. [4 + 2] Benzannulation of 3Alkenylpyrroles/Thiophenes with Propargylic Alcohols: Access to Substituted Indoles, Benzothiophenes, and Aza[5]helicenes. J. Org. Chem. 2017, 82, 2345-2354. (d) Petrone, D. A.; Isomura, M.; Franzoni, I.; Rössler, S. L.; Carreira, E. M. Allenylic Carbonates in Enantioselective IridiumCatalyzed Alkylations. J. Am. Chem. Soc. 2018, 140, 46974704. (e) Zhao, Y.; Jin, J.; Chan, P. W. H. Gold Catalyzed Photoredox C1-Alkynylation of N-Alkyl-1,2,3,4-tetrahydroisoquinolines by 1-Bromoalkynes with UVA LED Light. Adv. Synth. Catal. 2019, 361, 1313-1321. 
2) Bruker (2016). APEX3, SAINT and SADABS. Bruker AXS, Inc., Madison, Wisconsin, USA.

3) Sheldrick G. M. (2015).ActaCrystallogr C71: 3-8.

4) Muller, P, Herbst-Imer, R, Spek, A. L, Schneider, T. R, and Sawaya, M. R. Crystal Structure Refinement: A Crystallographer's Guide to SHELXL. Muller, P. Ed. 2006 Oxford University Press: Oxford, New York, pp. 57-91. 
7) ${ }^{1} \mathrm{H}$ NMR and ${ }^{13} \mathrm{C}$ NMR spectral copies of

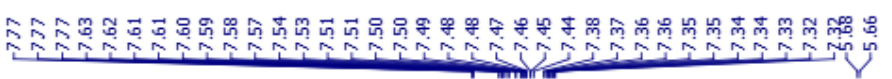

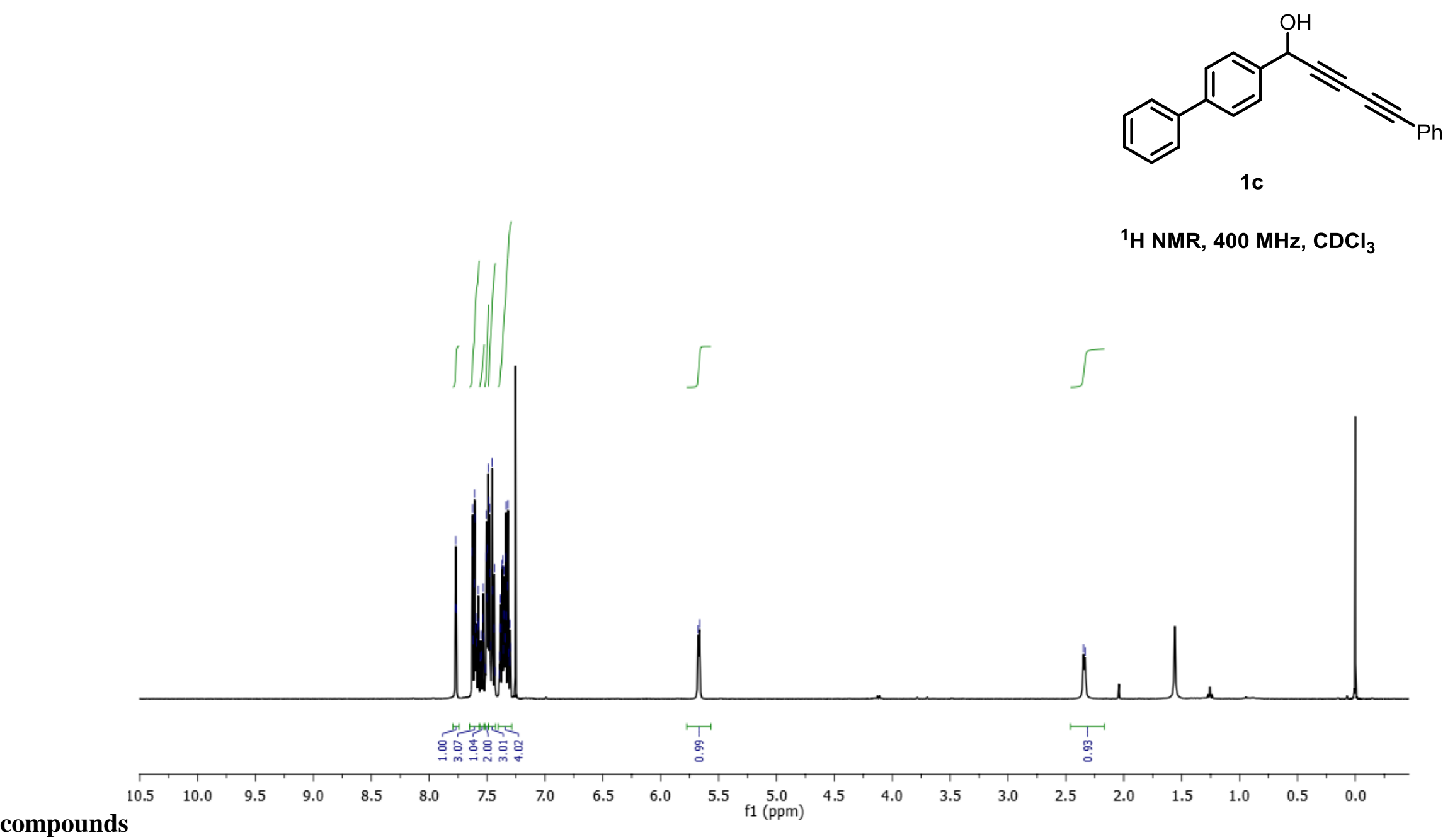

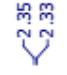

${ }^{1} \mathrm{H} \mathrm{NMR}, 400 \mathrm{MHz}, \mathrm{CDCl}_{3}$

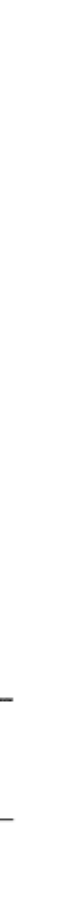




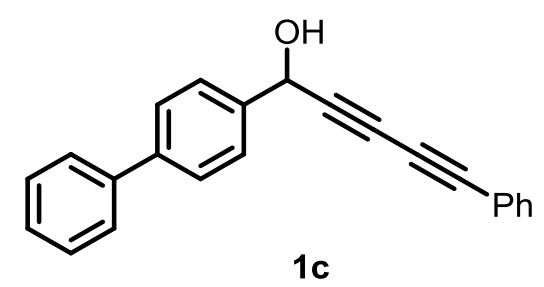

${ }^{13} \mathrm{C}$ NMR, $125 \mathrm{MHz}, \mathrm{CDCl}_{3}$

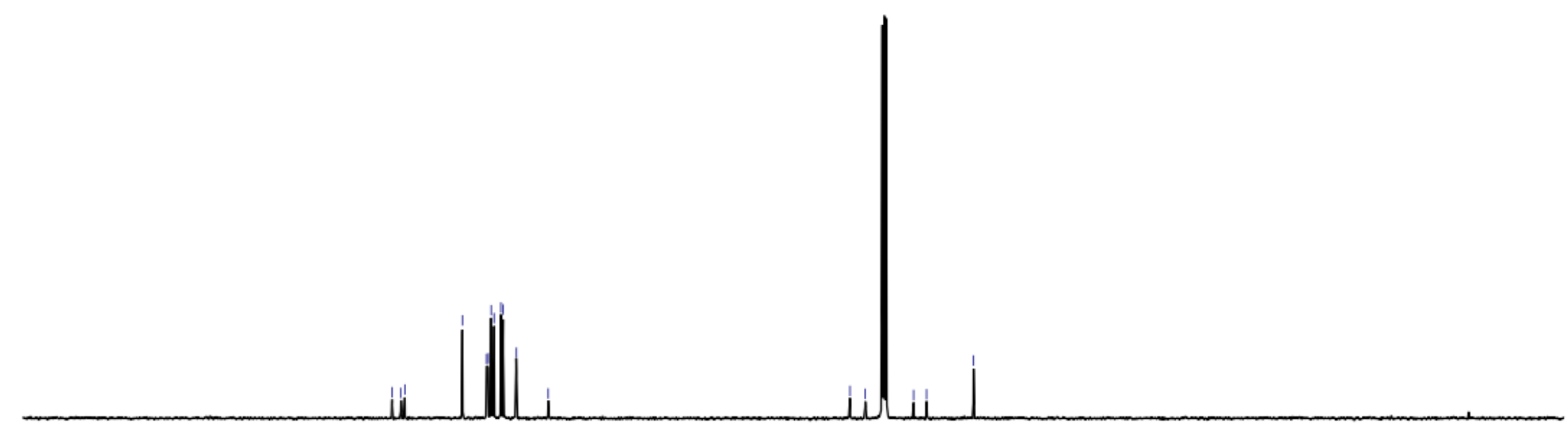




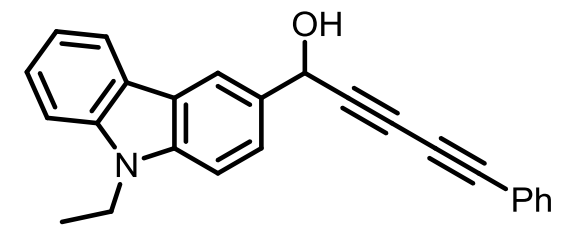

$1 e$

${ }^{1} \mathrm{H}$ NMR, $400 \mathrm{MHz}, \mathrm{CDCl}_{3}$
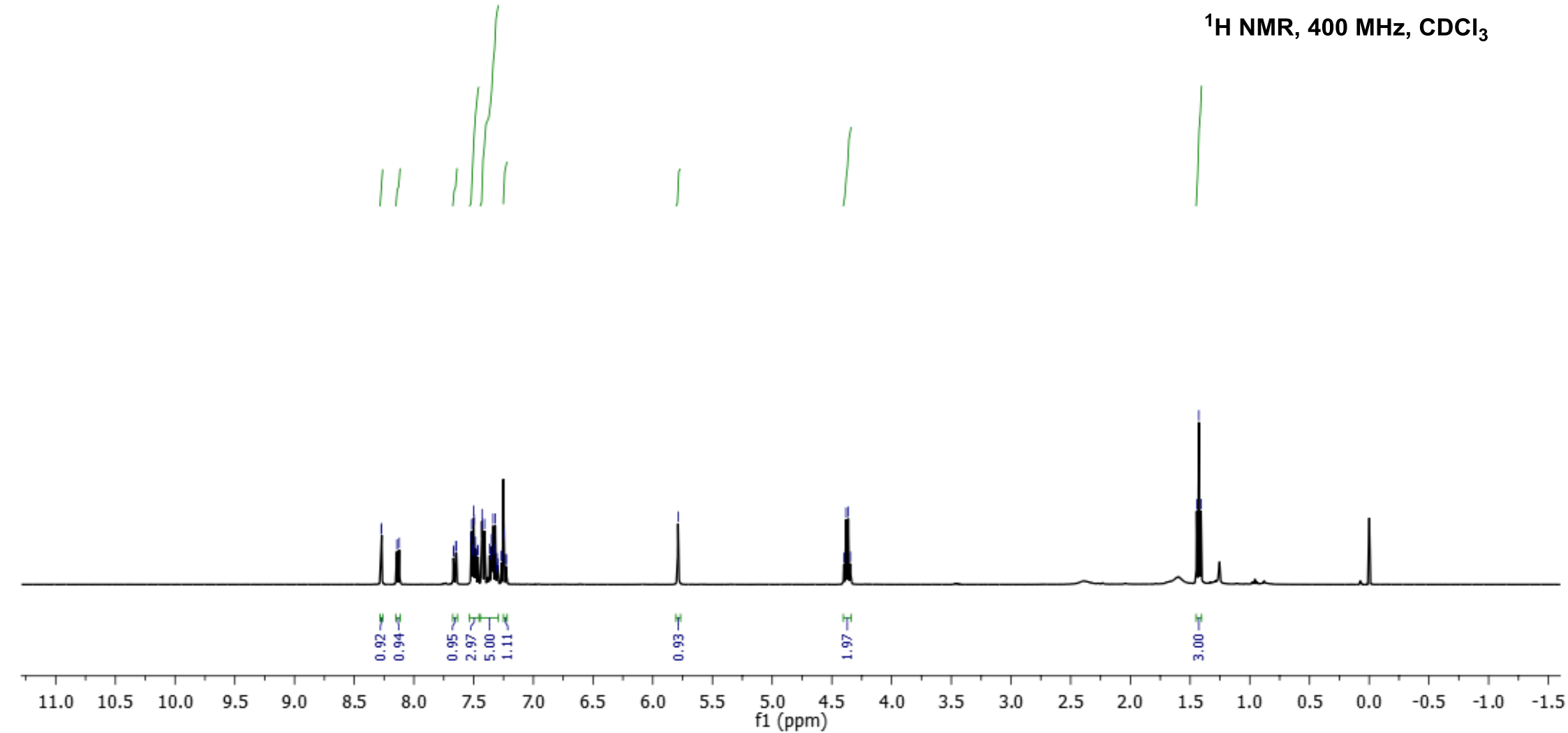


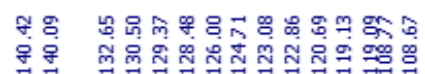

†ో

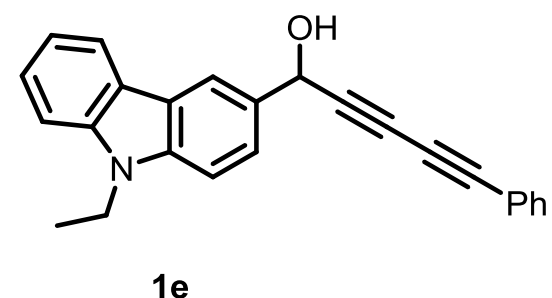

${ }^{13} \mathrm{C} \mathrm{NMR}, 101 \mathrm{MHz}, \mathrm{CDCl}_{3}$
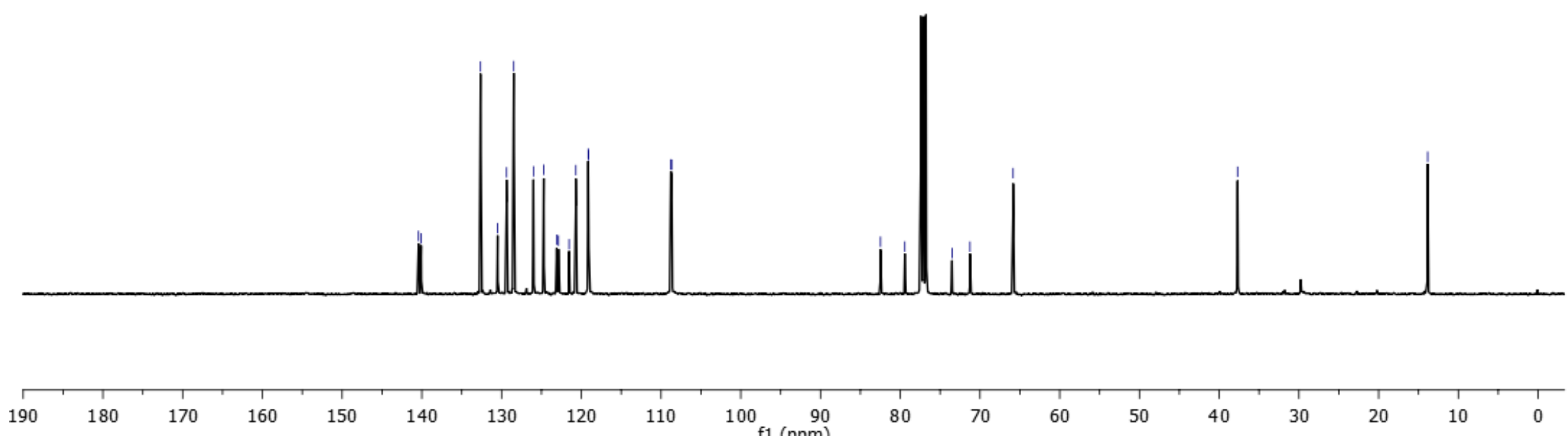
f1 (ppm) 


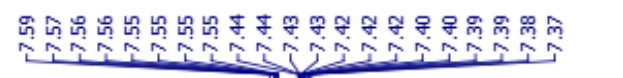

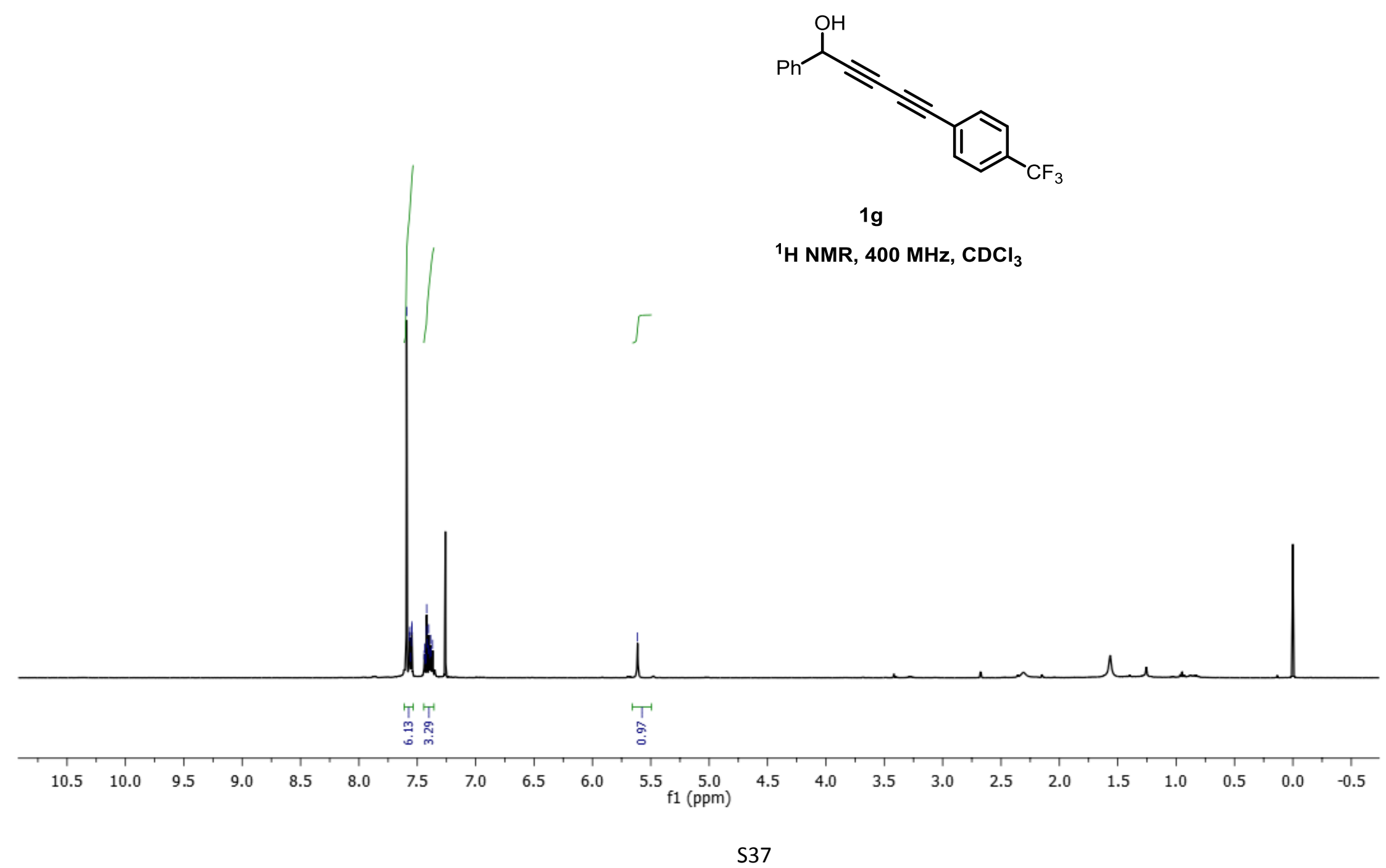




$$
I=
$$




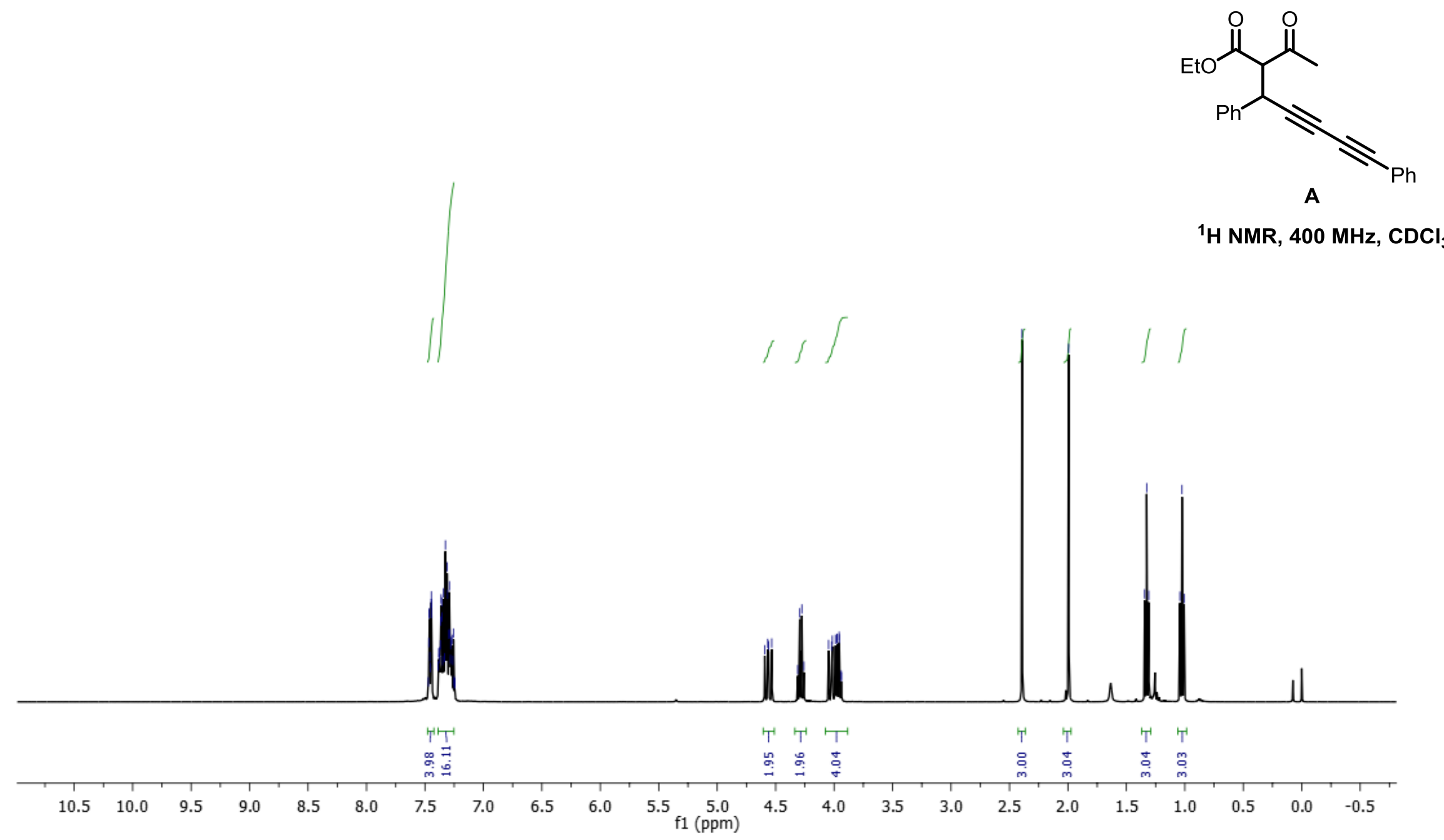



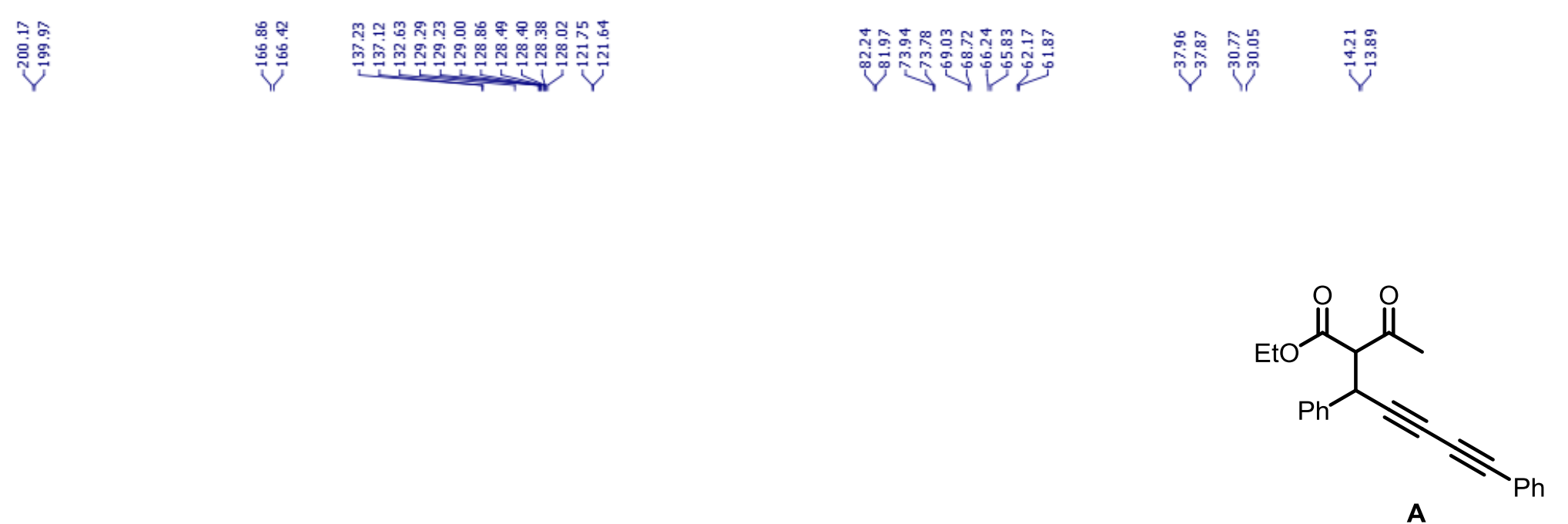

$13 \mathrm{NMR}, 100 \mathrm{MHz}, \mathrm{CDCl}_{3}$

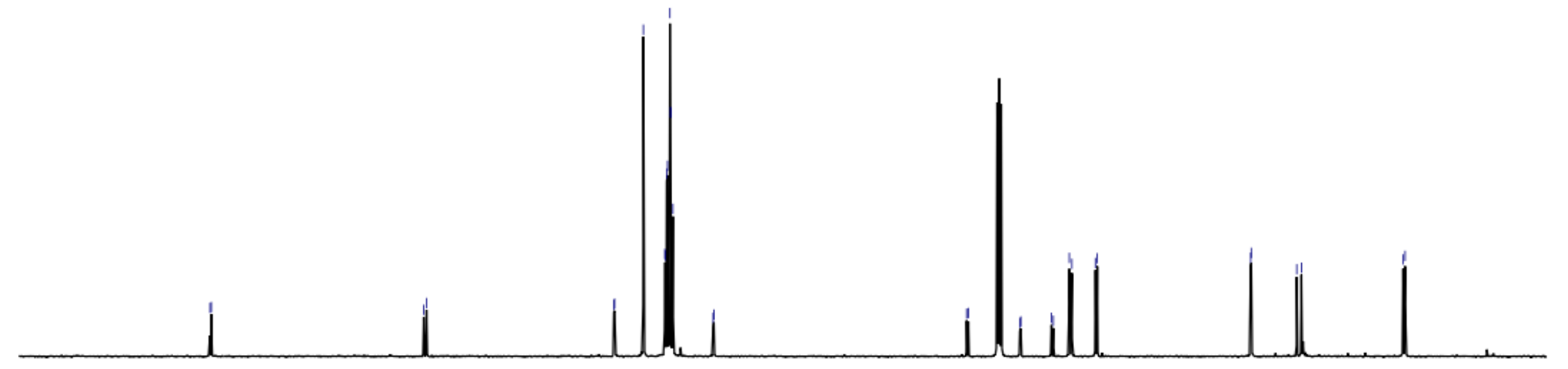

$\begin{array}{lllllllllll}220 & 210 & 200 & 190 & 180 & 170 & 160 & 150 & 140 & 130 & 120 \\ \mathrm{f} 1(\mathrm{ppm})\end{array}$ 


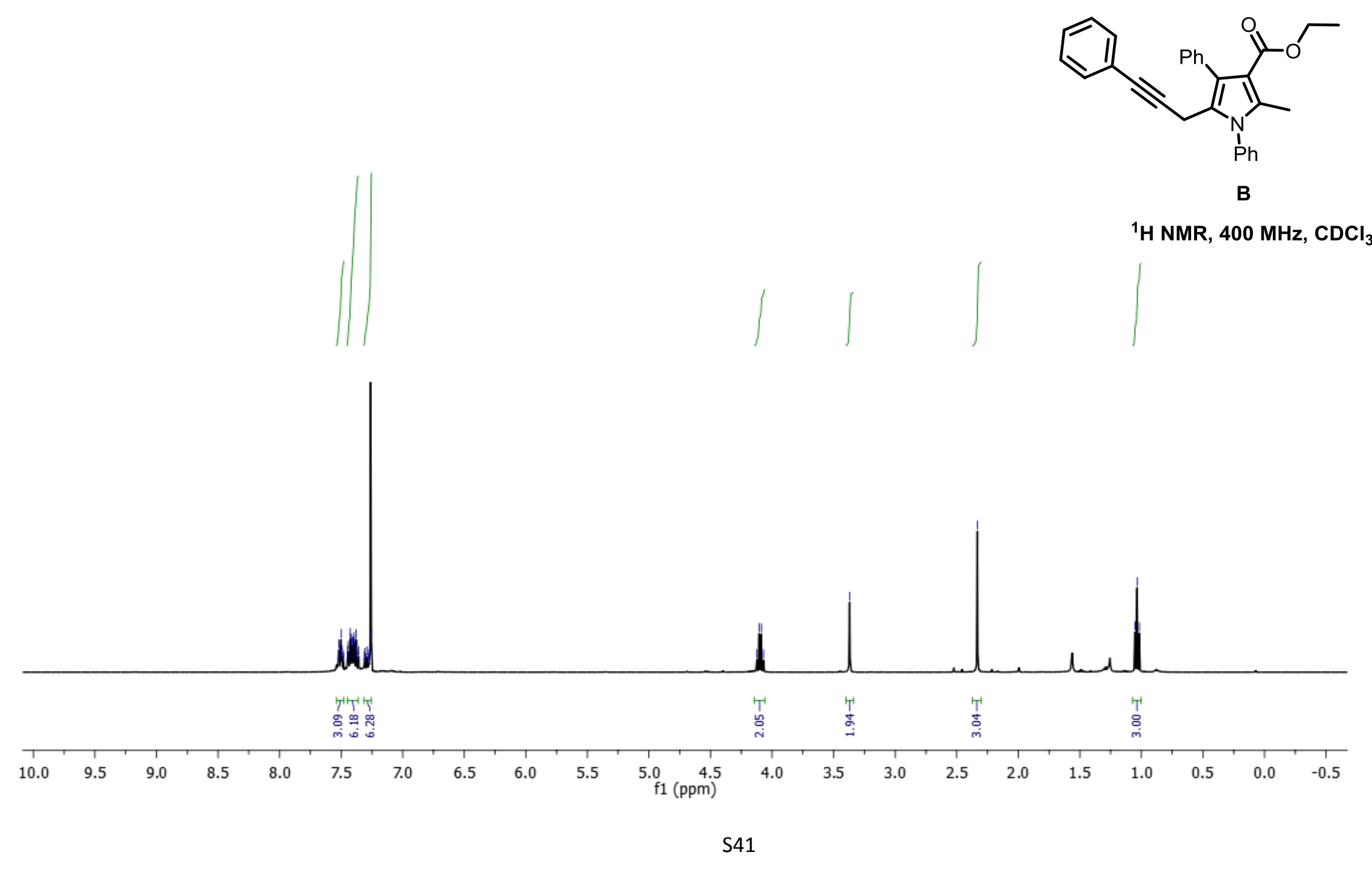




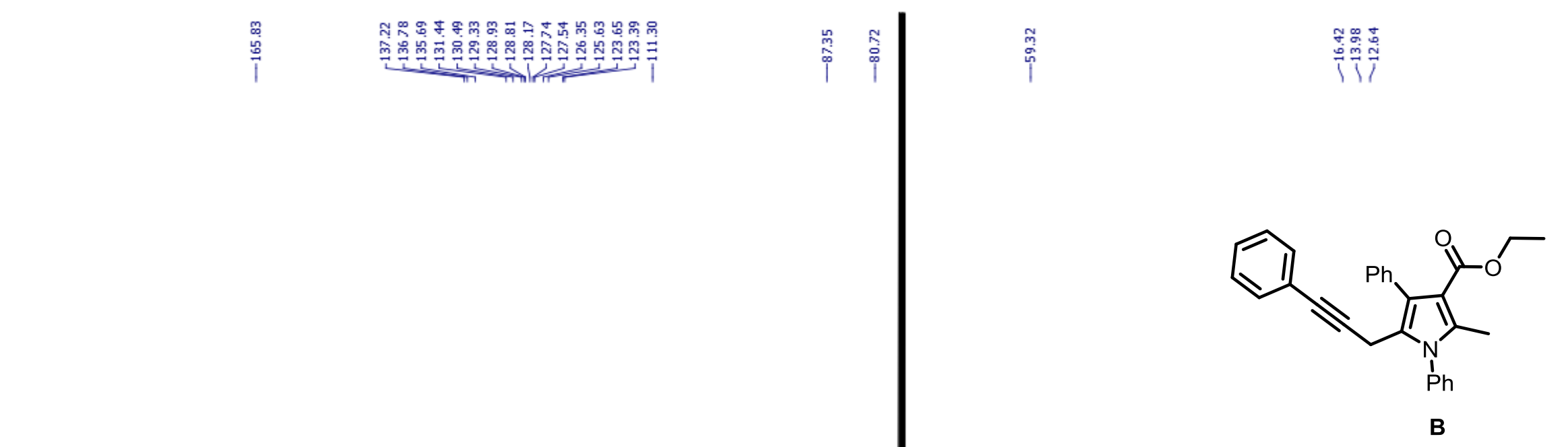

${ }^{13} \mathrm{C} \mathrm{NMR}, 100 \mathrm{MHz}, \mathrm{CDCl}_{3}$
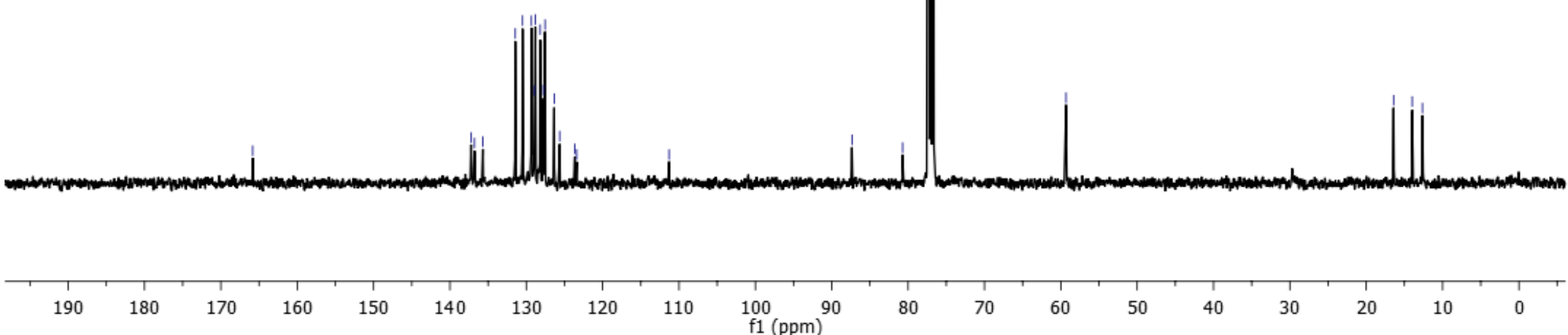


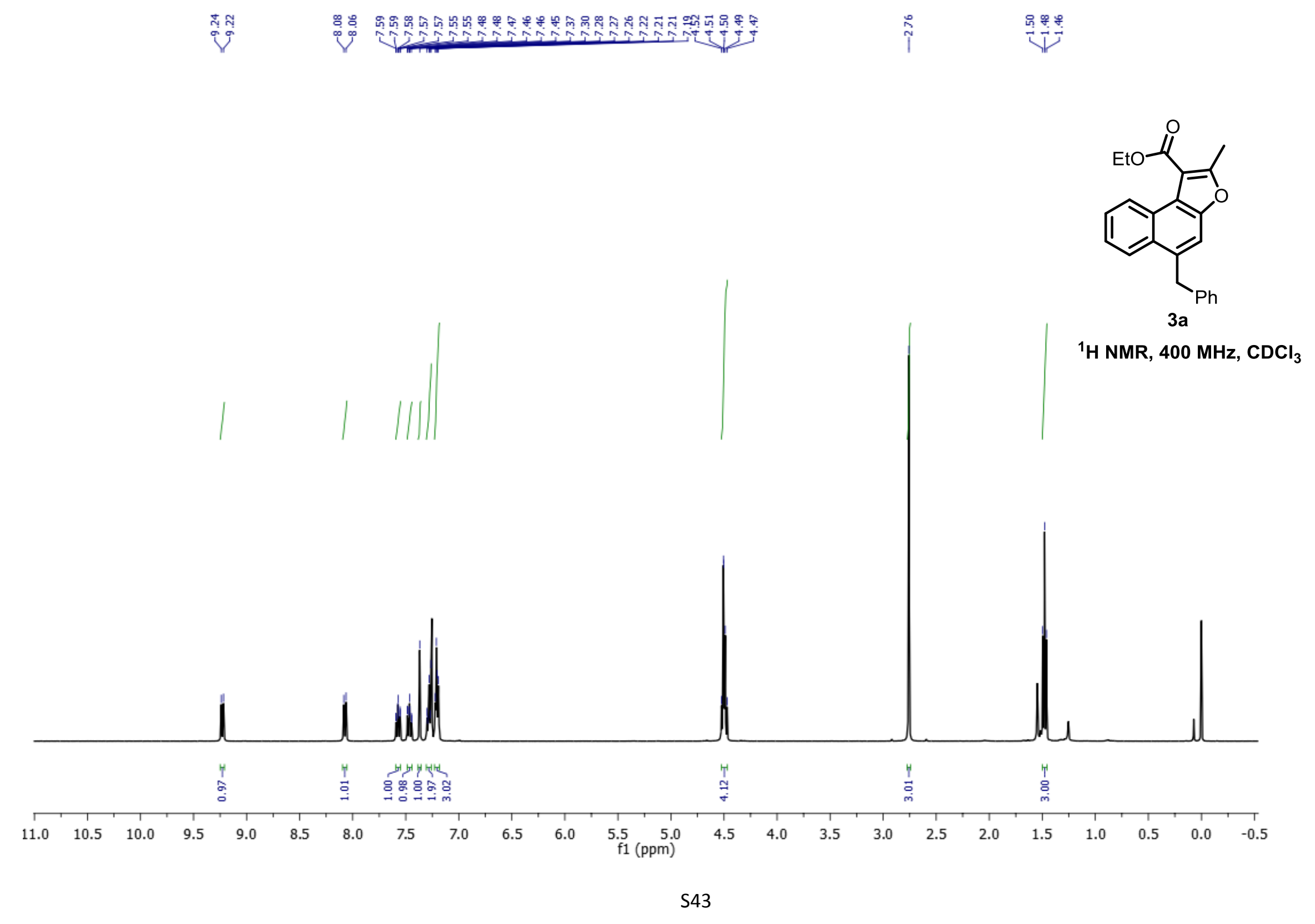




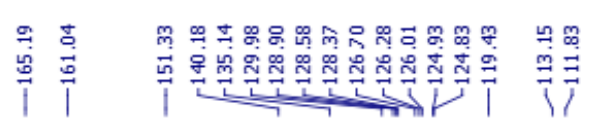

$\infty$
0
0
0

菖

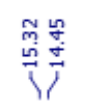

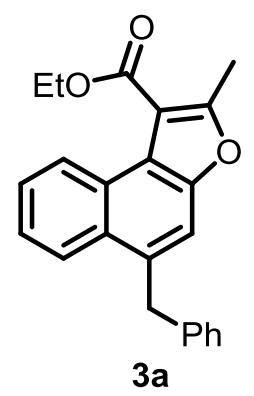

${ }^{13} \mathrm{C}$ NMR, $125 \mathrm{MHz}, \mathrm{CDCl}_{3}$

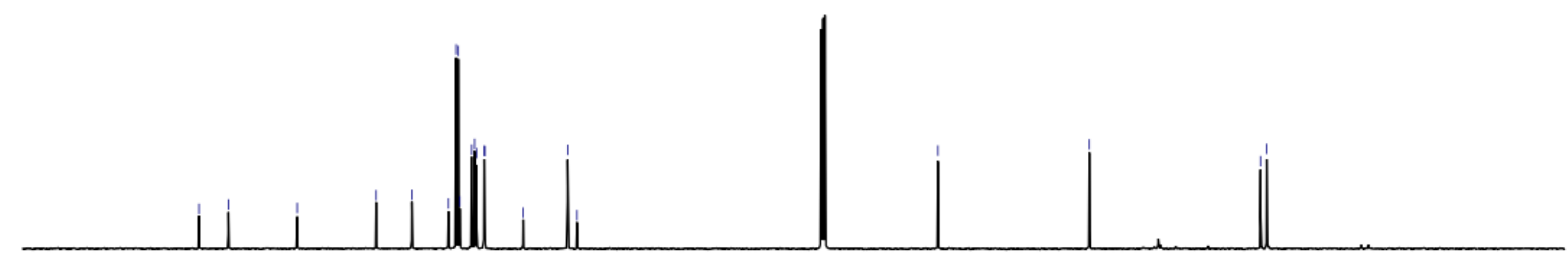

190

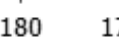

$170 \quad 160$
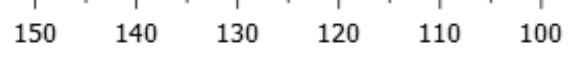

$90 \begin{gathered}80 \\ \mathrm{f} 1(\mathrm{ppm})\end{gathered}$ 


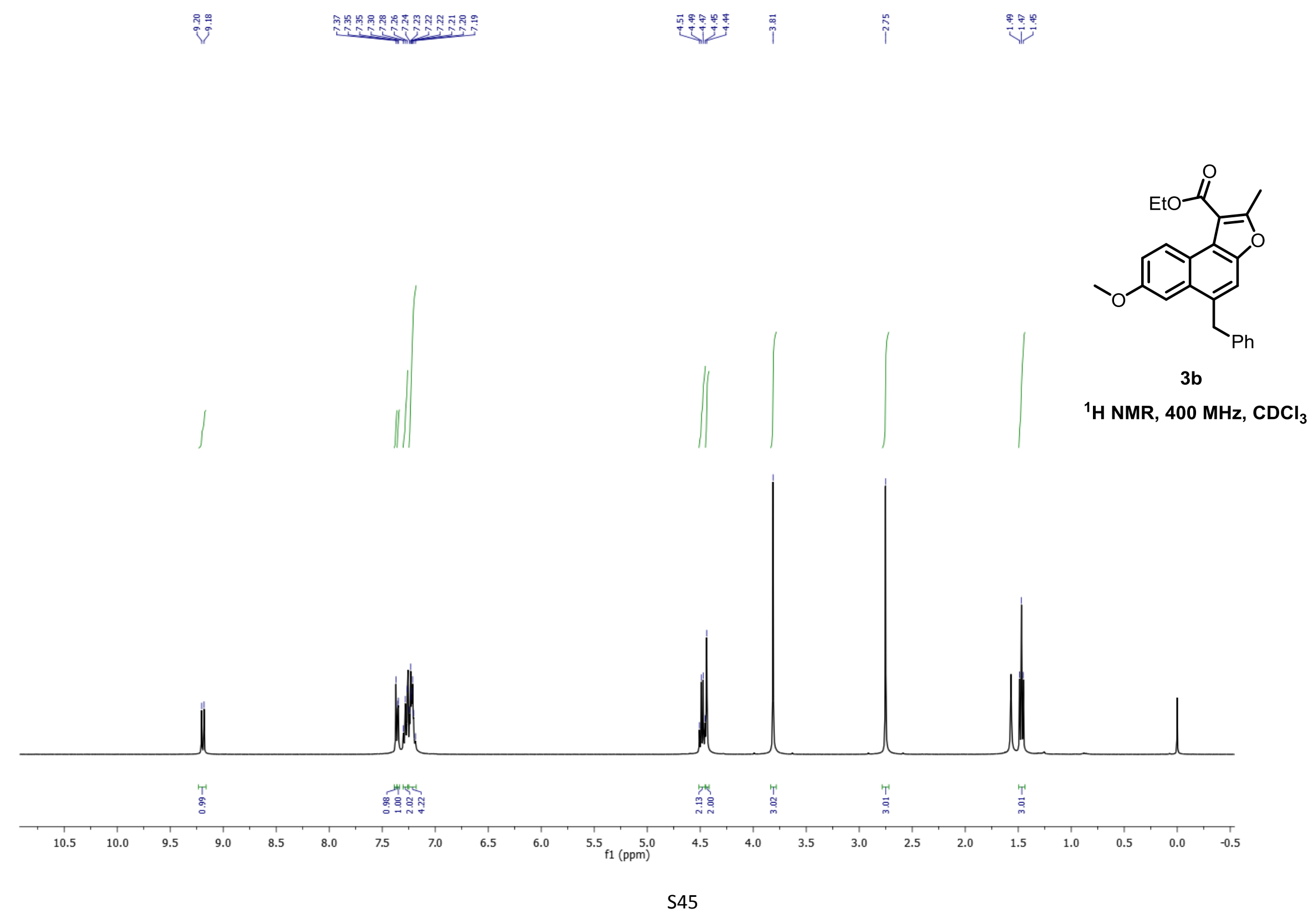




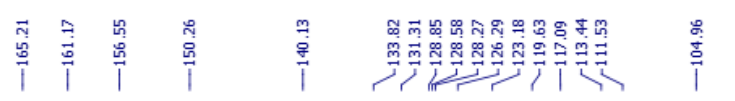

辛市

琹重

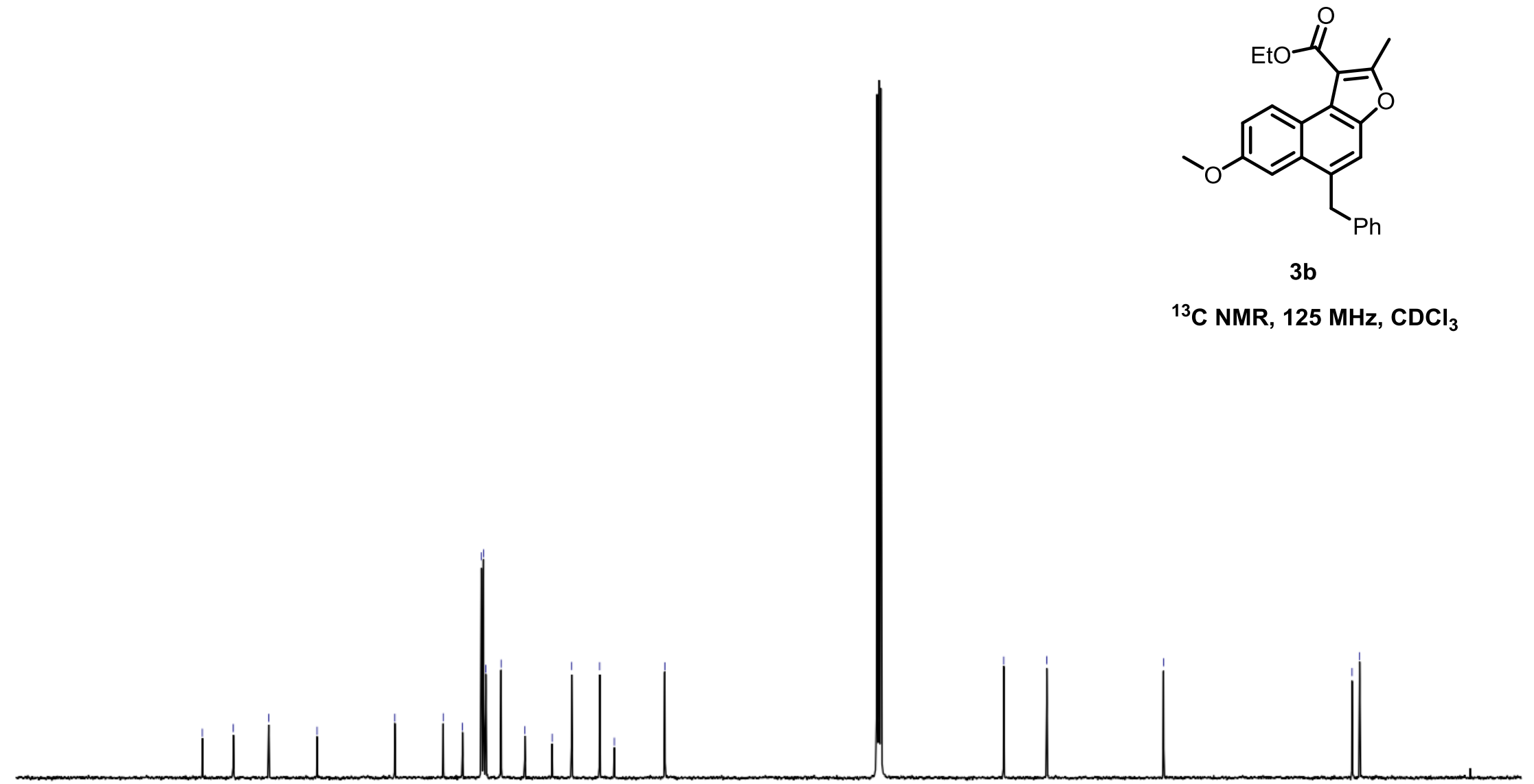

$3 b$

${ }^{13} \mathrm{C}$ NMR, $125 \mathrm{MHz}, \mathrm{CDCl}_{3}$ 


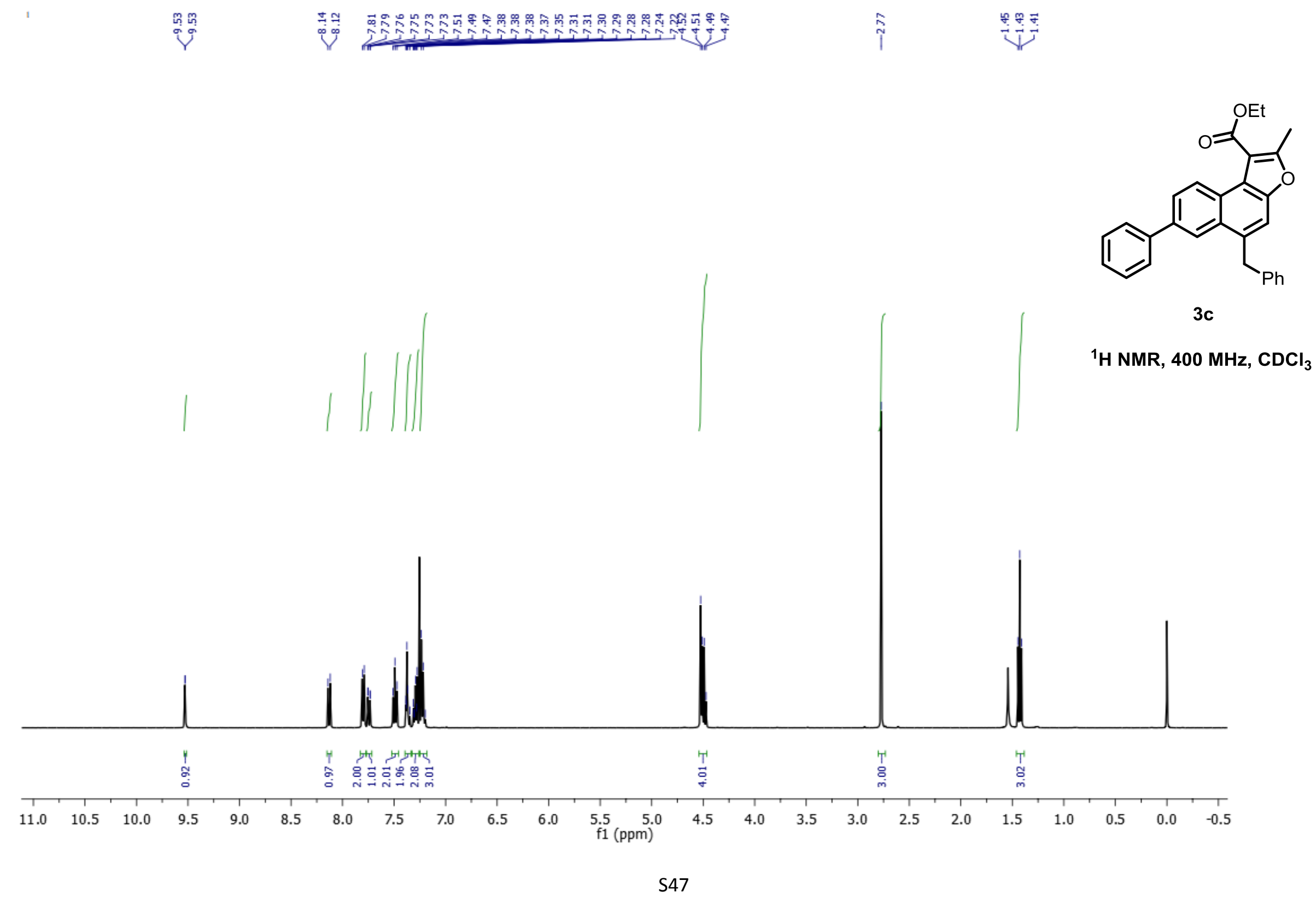




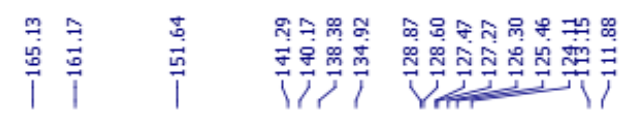

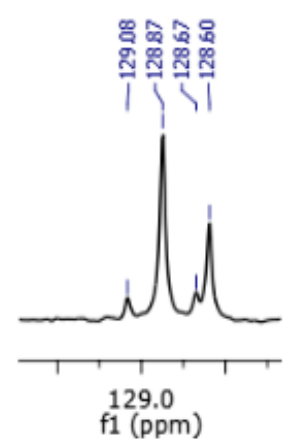

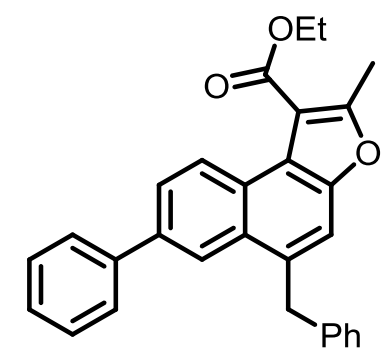

$3 c$

${ }^{13} \mathrm{C}$ NMR, $100 \mathrm{MHz}, \mathrm{CDCl}_{3}$

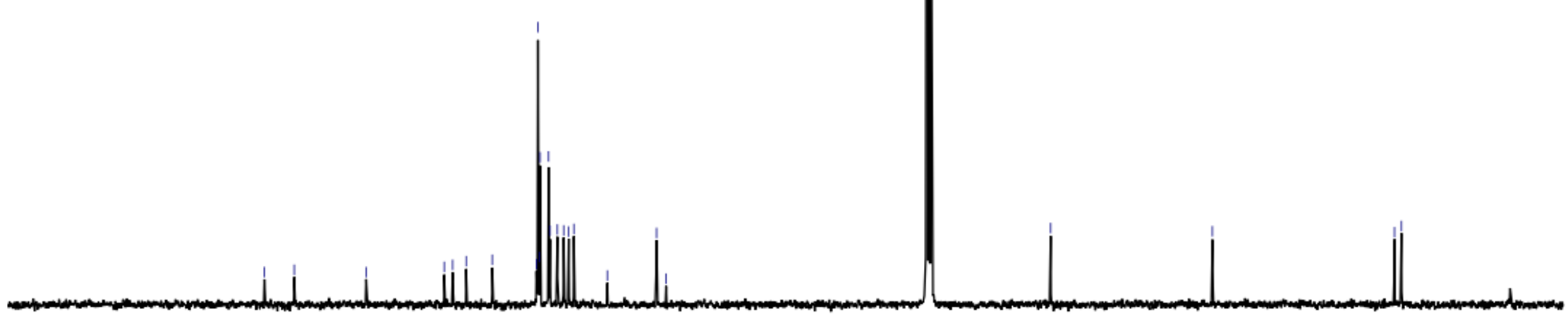




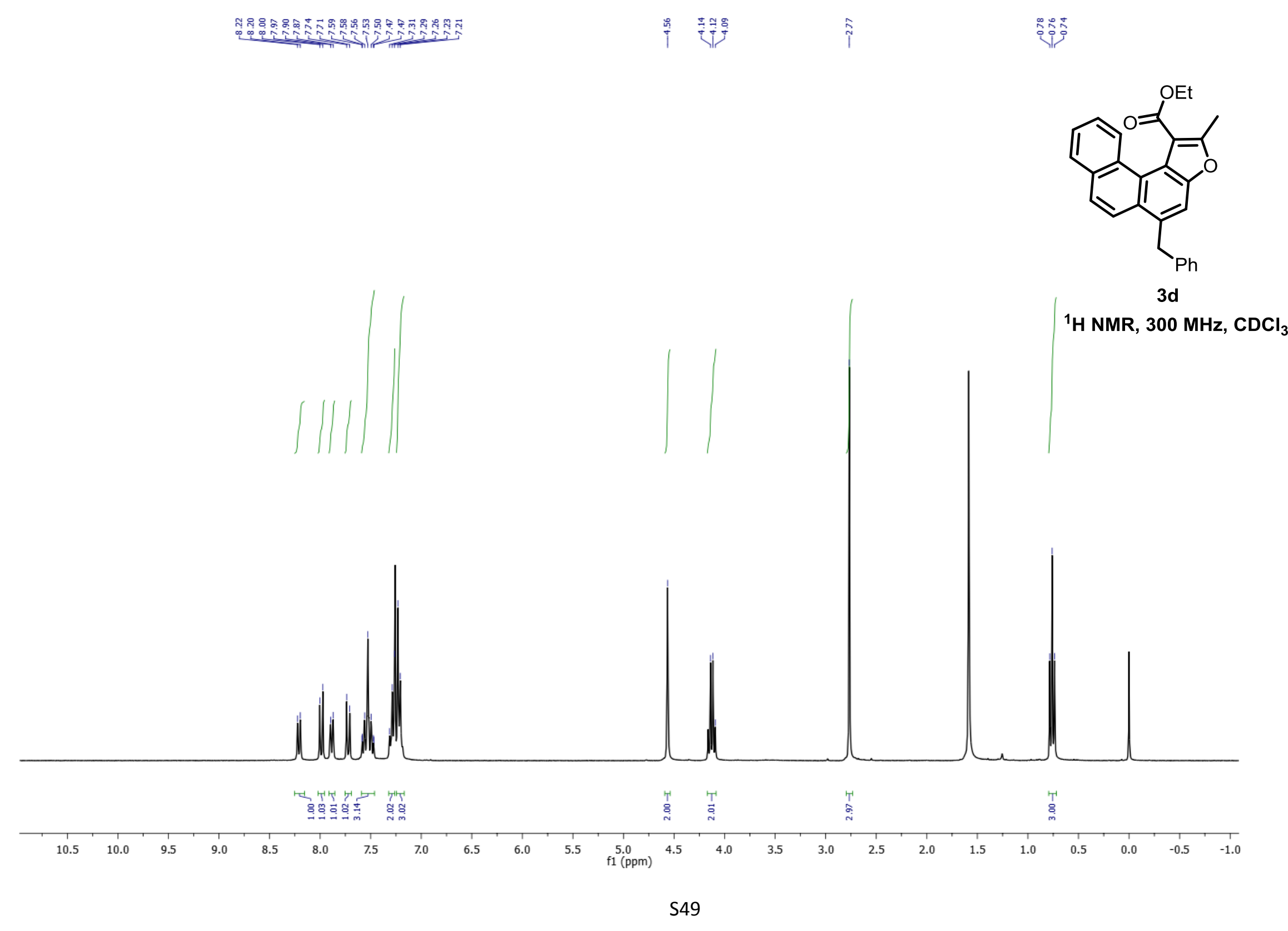




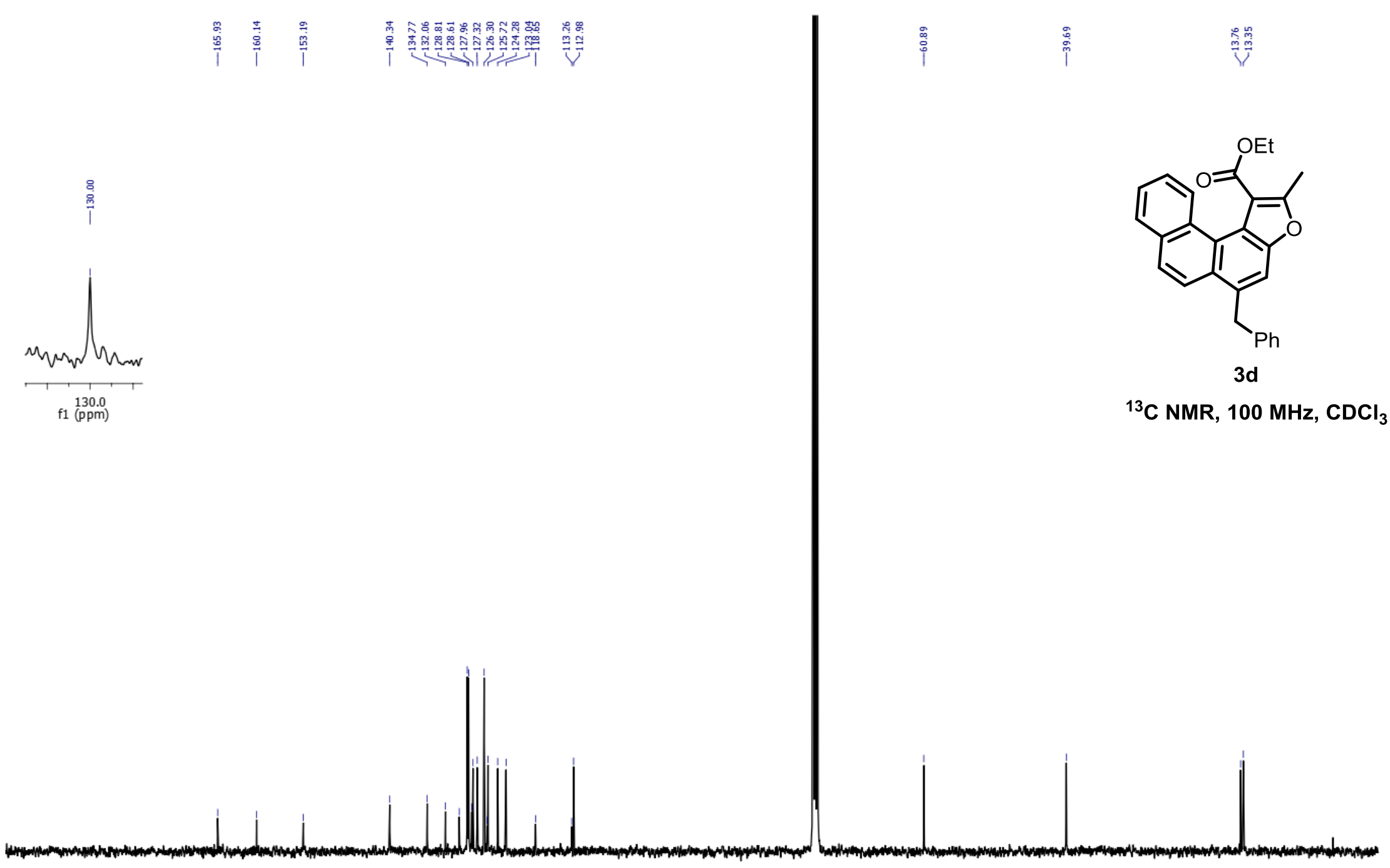




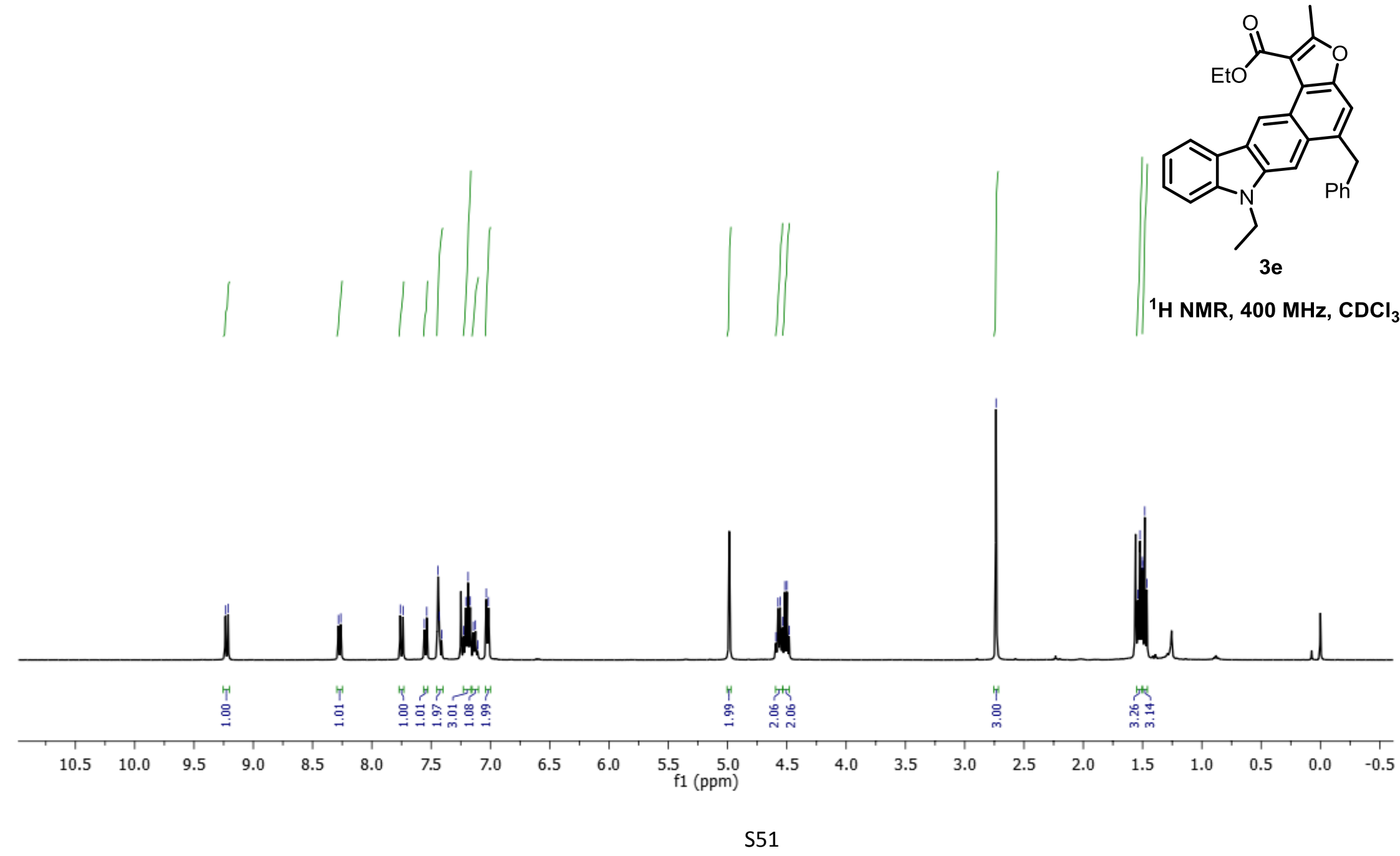




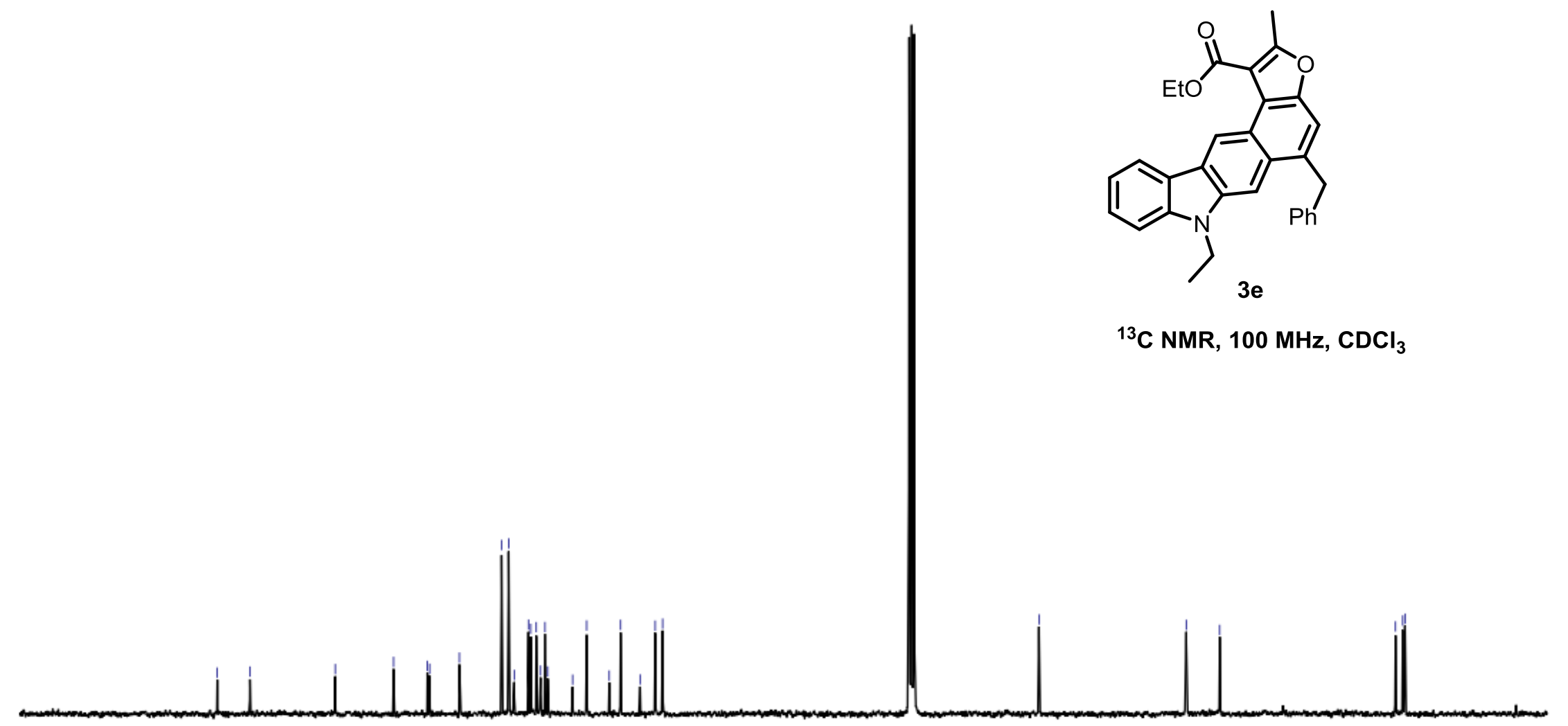

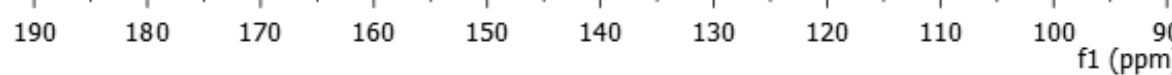




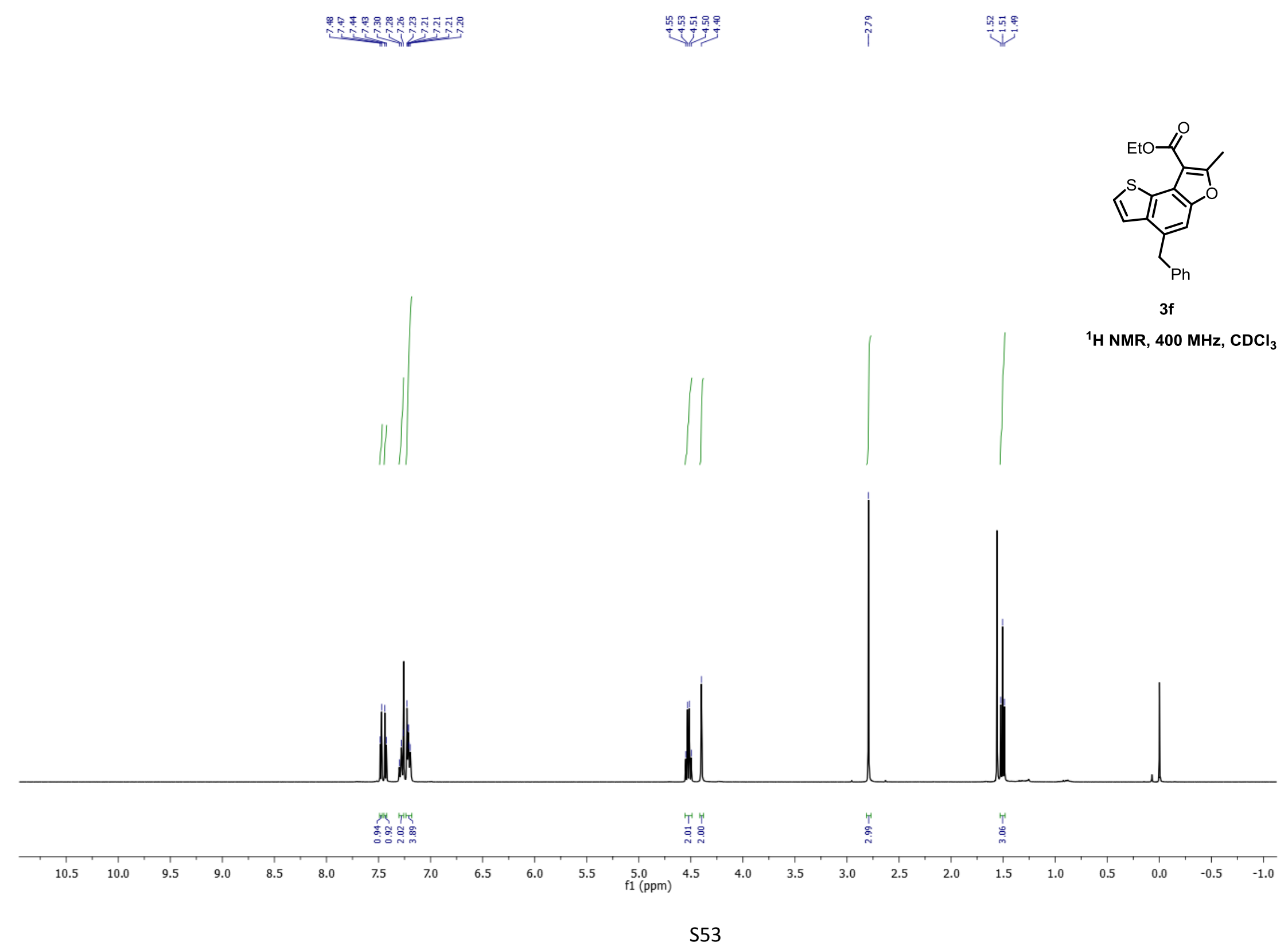




$$
\text { ئ }
$$




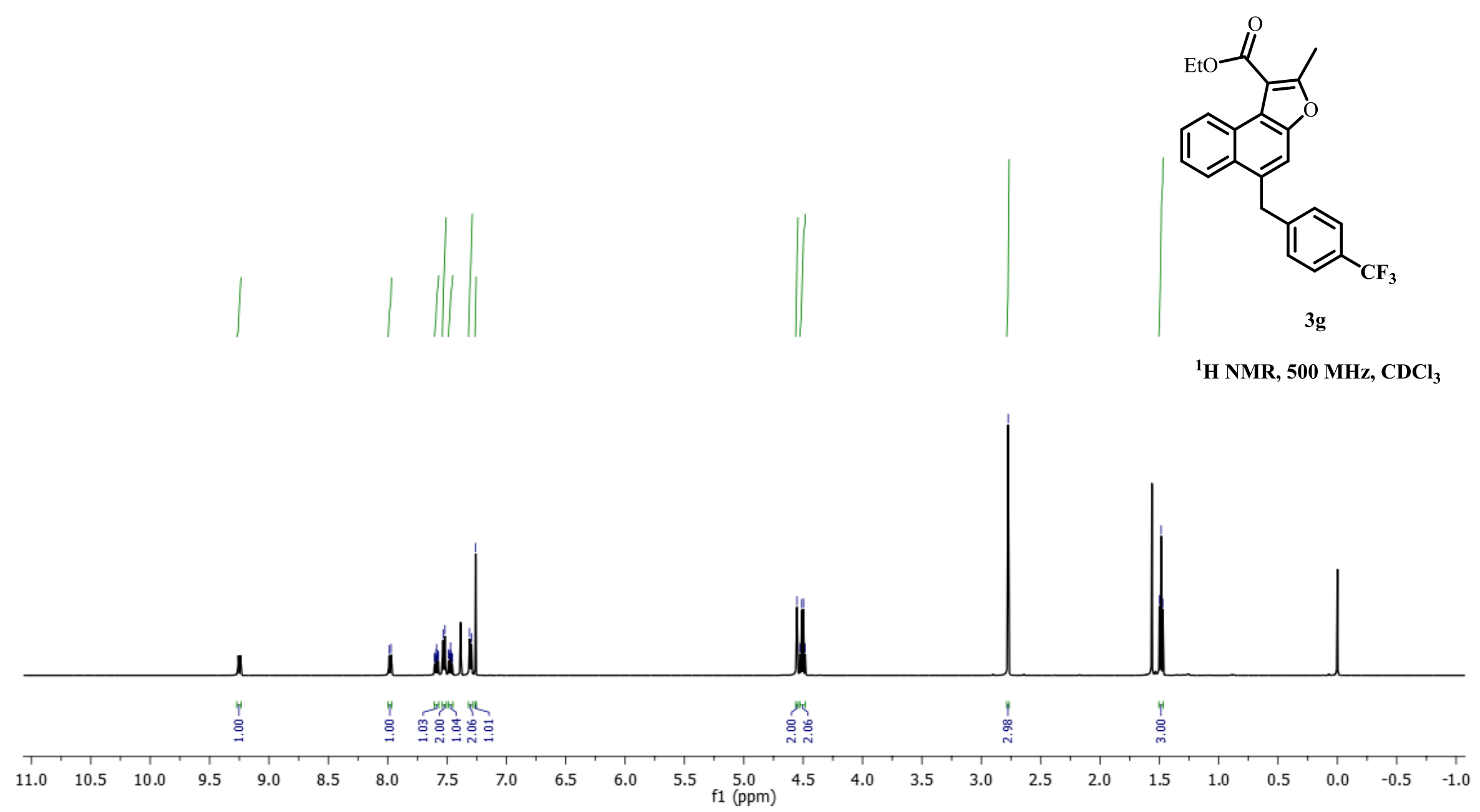




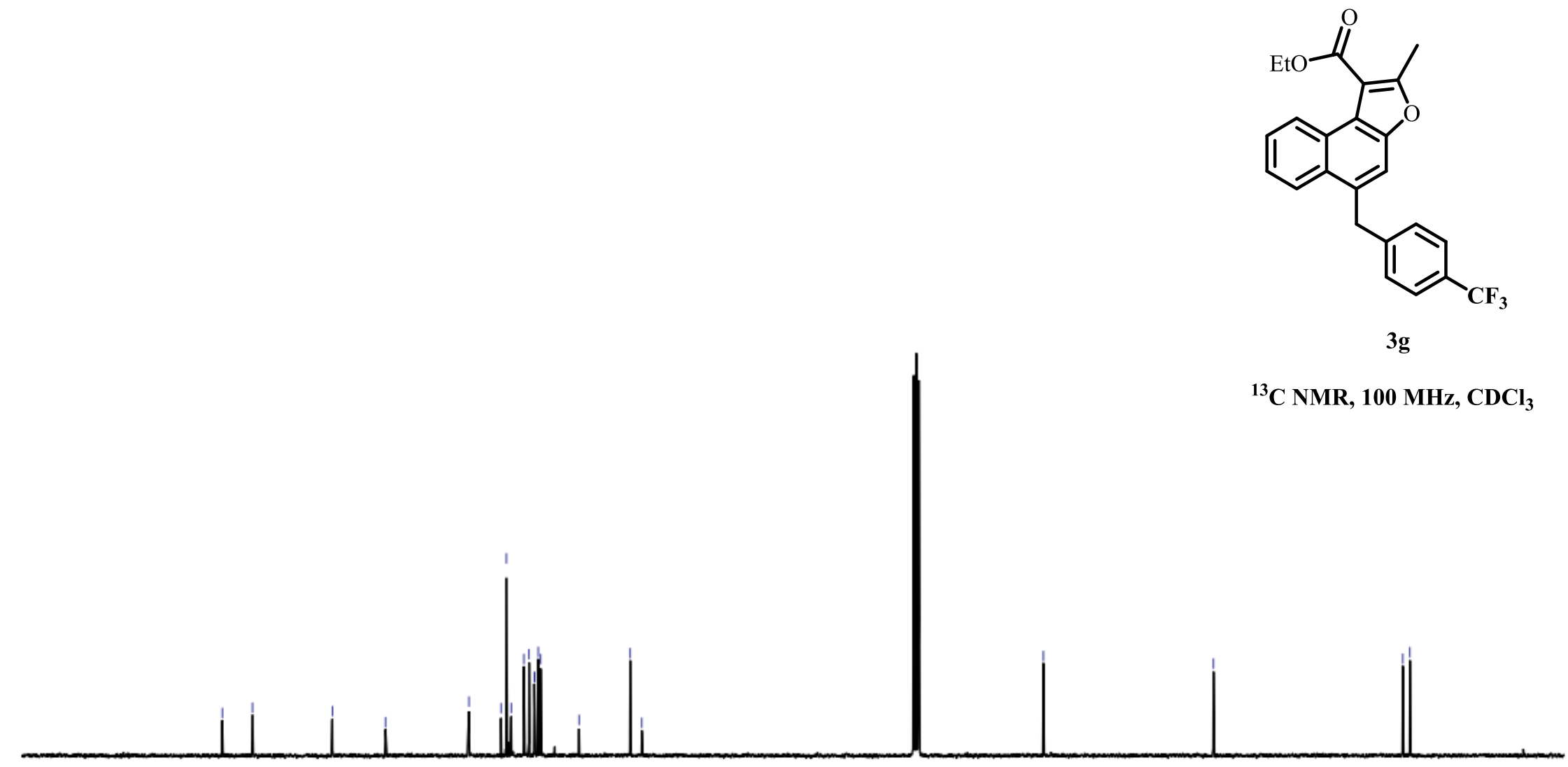

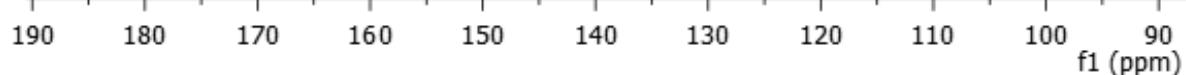




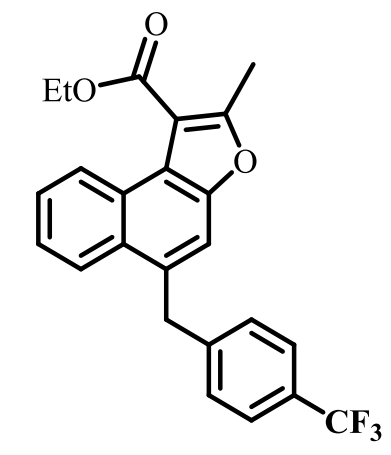

$3 g$

${ }^{19} \mathrm{~F}$ NMR, $377 \mathrm{MHz}, \mathrm{CDCl}_{3}$

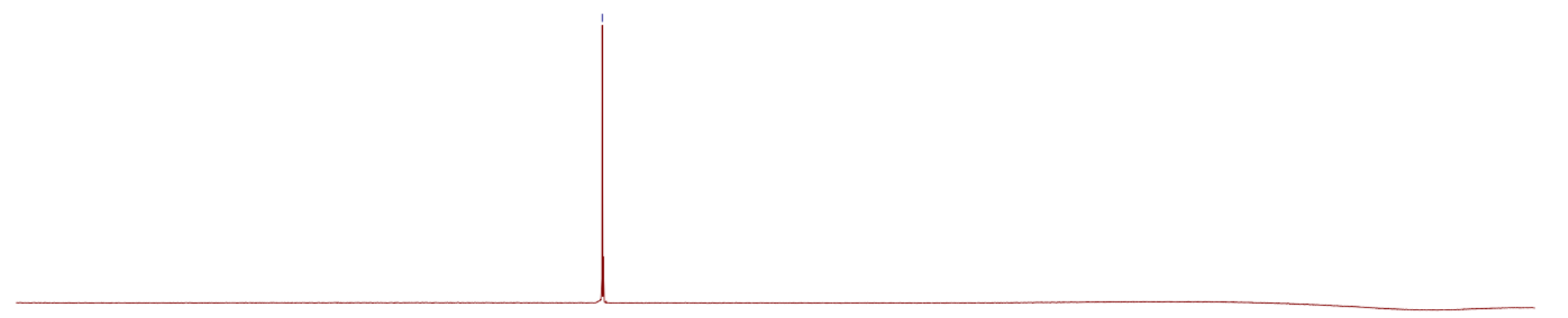

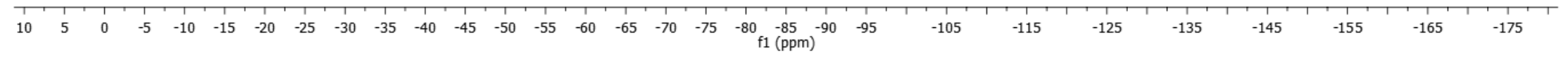




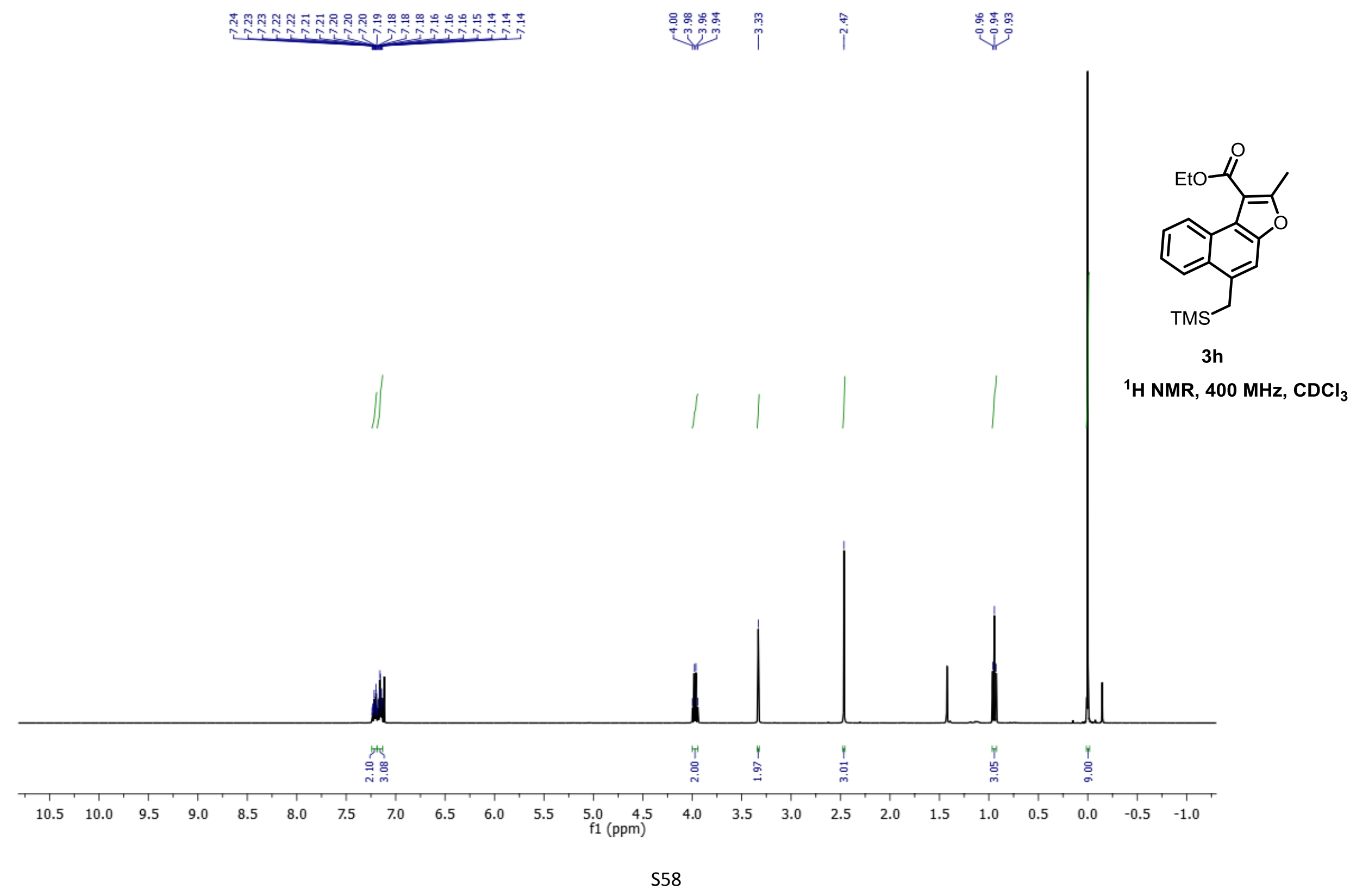




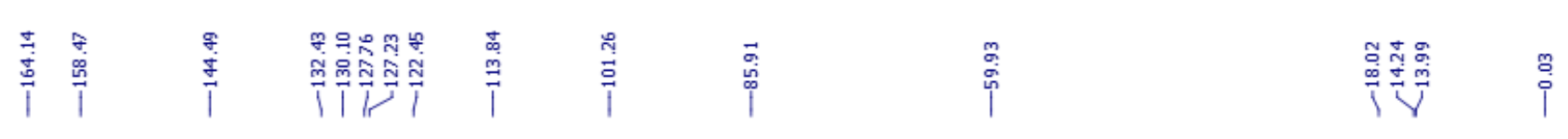

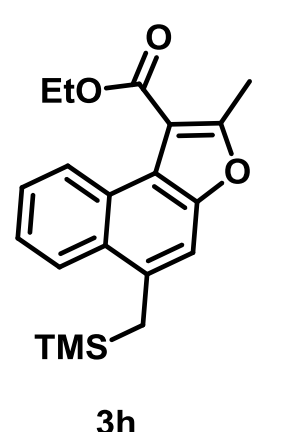

${ }^{13} \mathrm{C} \mathrm{NMR}, 100 \mathrm{MHz}, \mathrm{CDCl}_{3}$

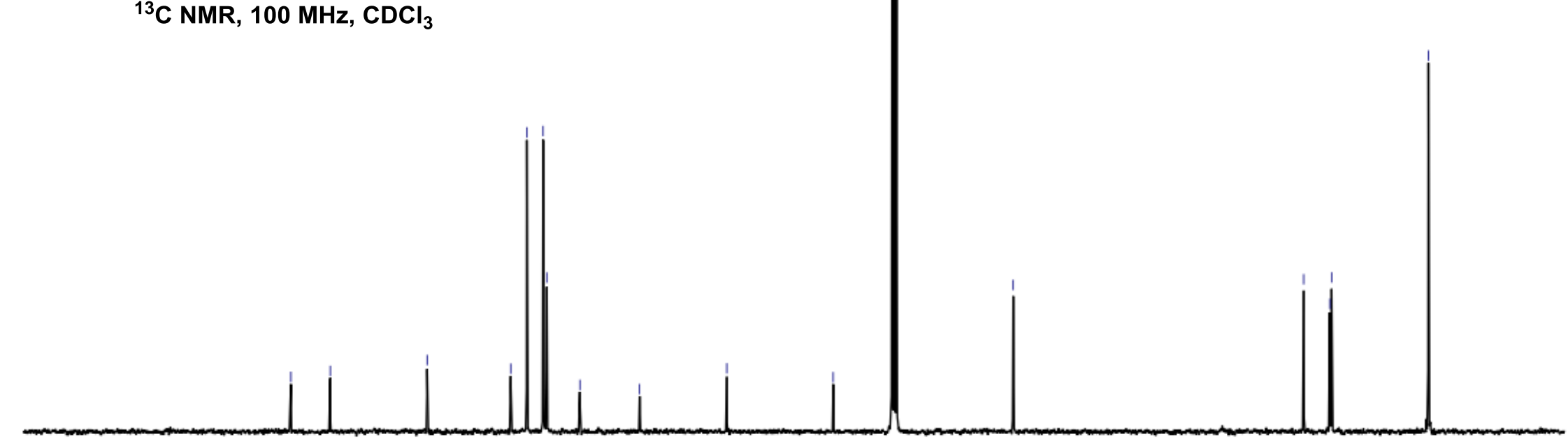

$\begin{array}{lllllllllll}200 & 190 & 180 & 170 & 160 & 150 & 140 & 130 & 120 & 110 & 100 \\ \mathrm{f} 1(\mathrm{ppm})\end{array}$ 


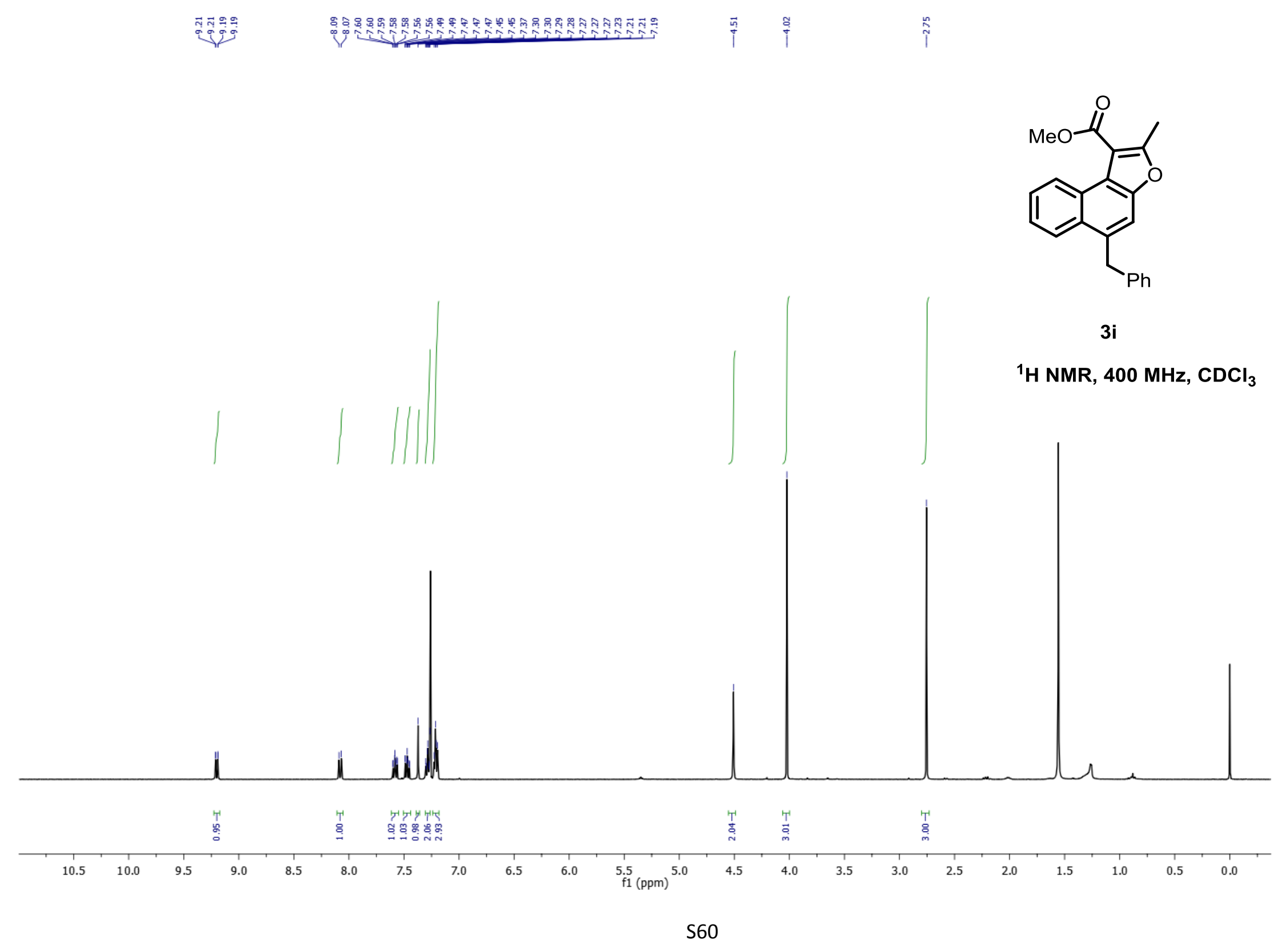




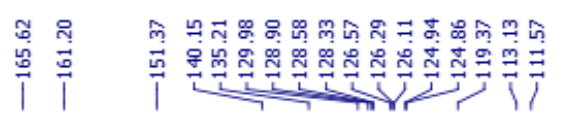

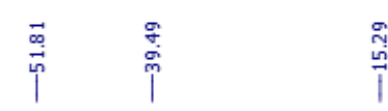

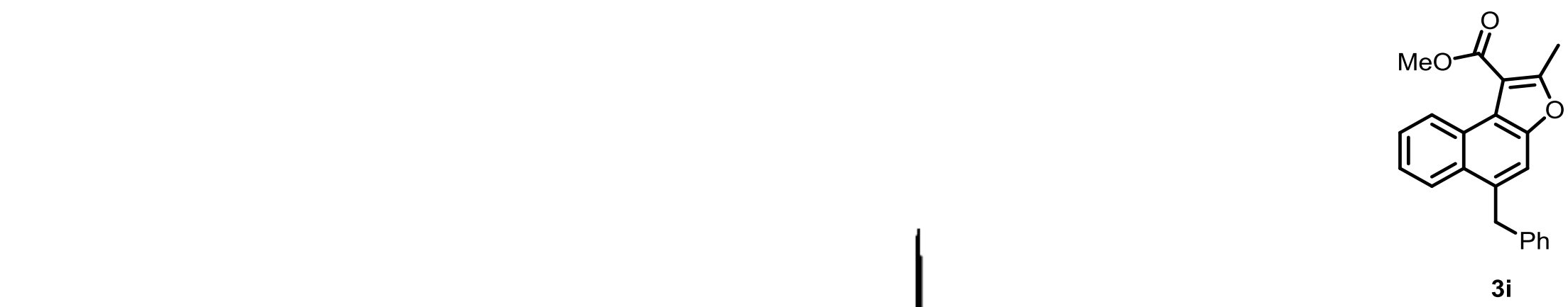

${ }^{13} \mathrm{C}$ NMR, $100 \mathrm{MHz}, \mathrm{CDCl}_{3}$

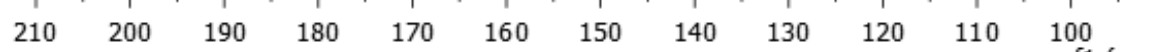
$\begin{array}{rr}100 & 90 \\ \mathrm{f} 1(\mathrm{ppm})\end{array}$ 


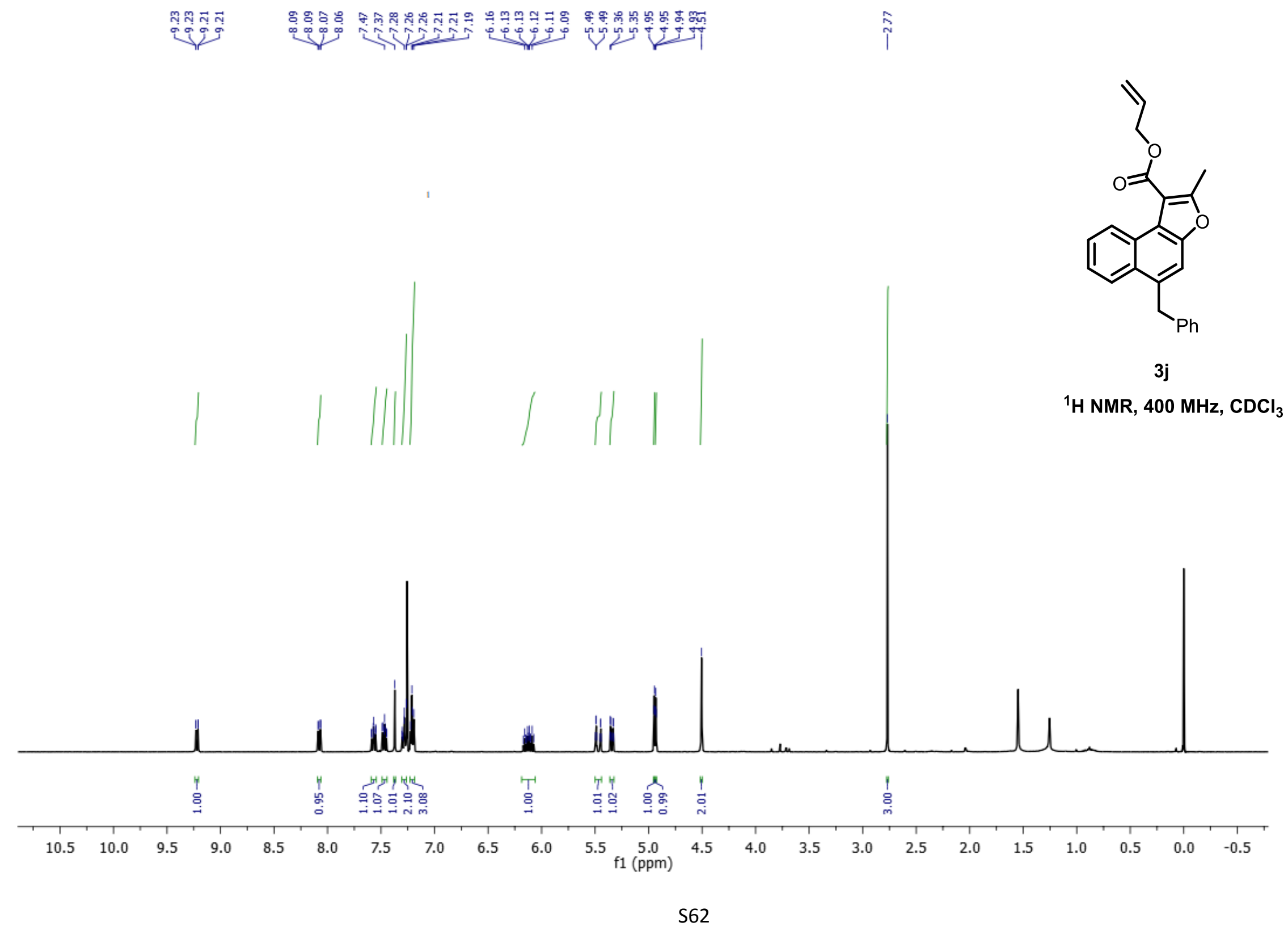




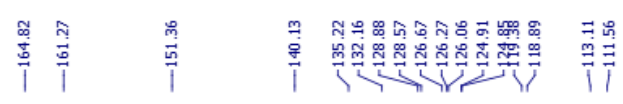
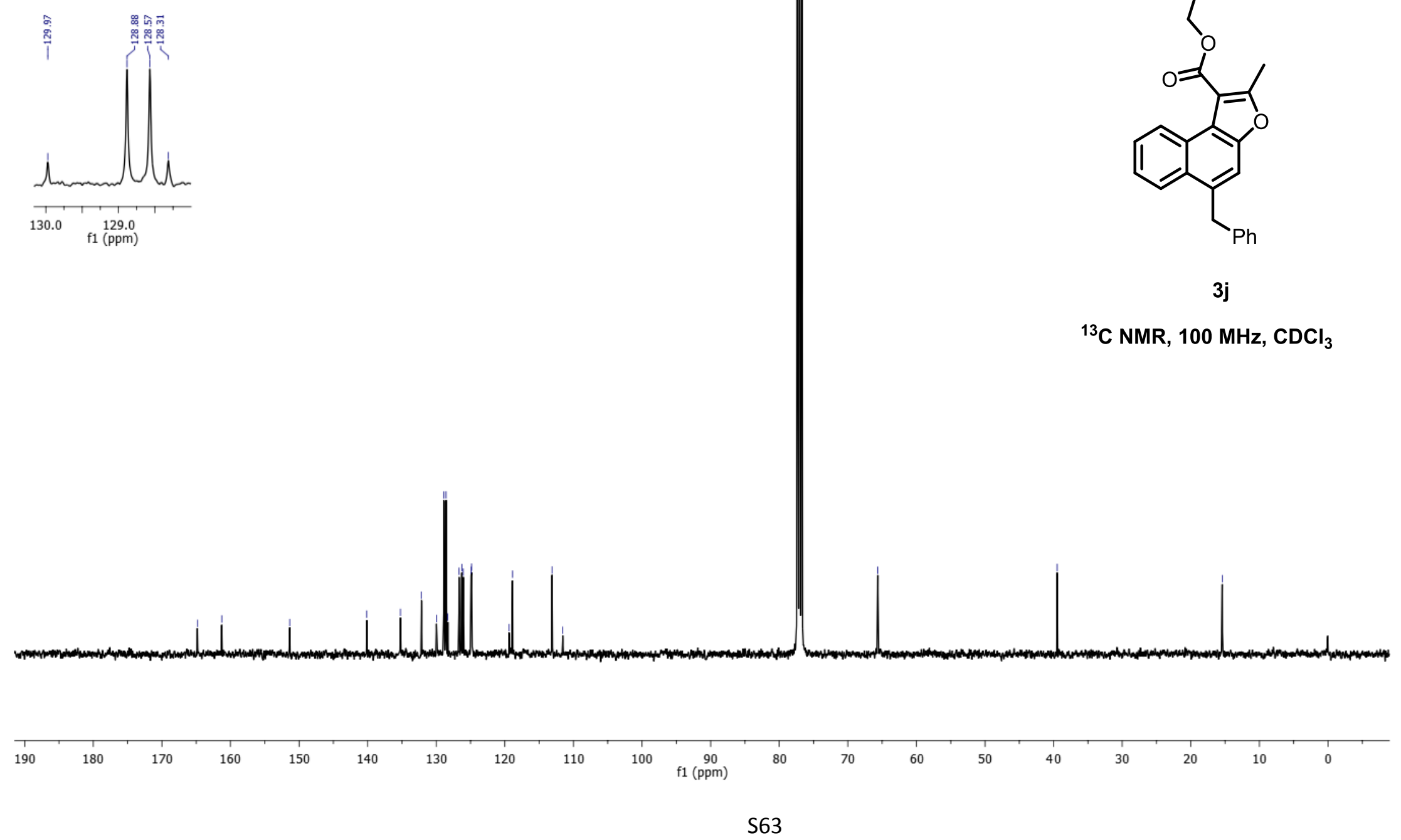

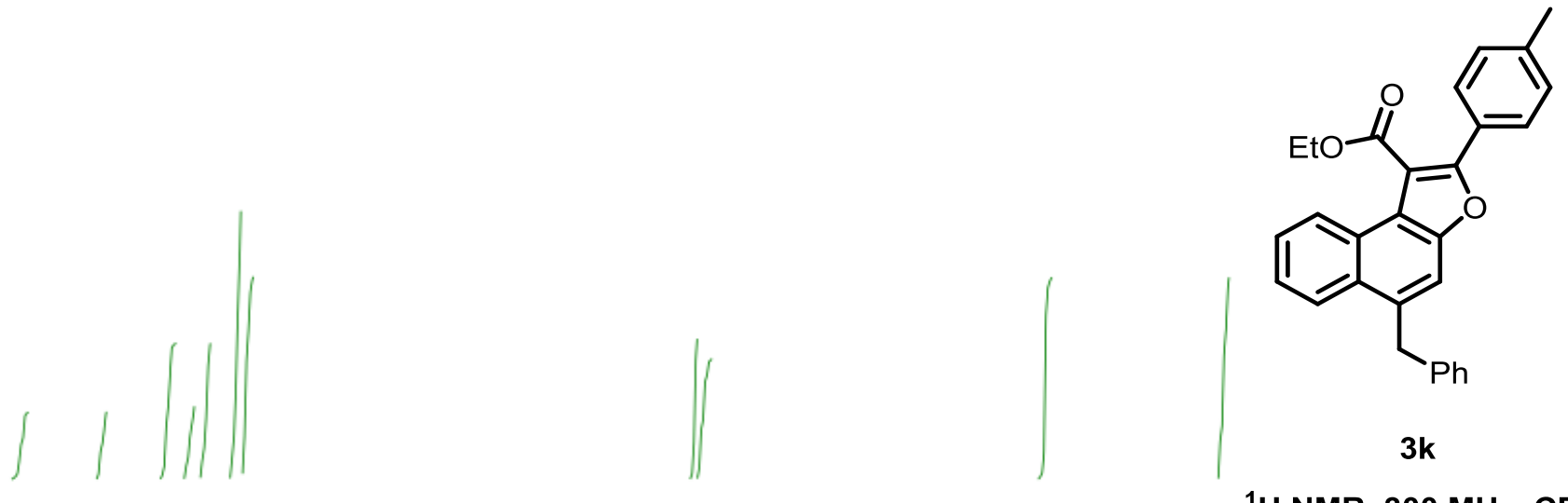

${ }^{1} \mathrm{H}$ NMR, $300 \mathrm{MHz}, \mathrm{CDCl}_{3}$

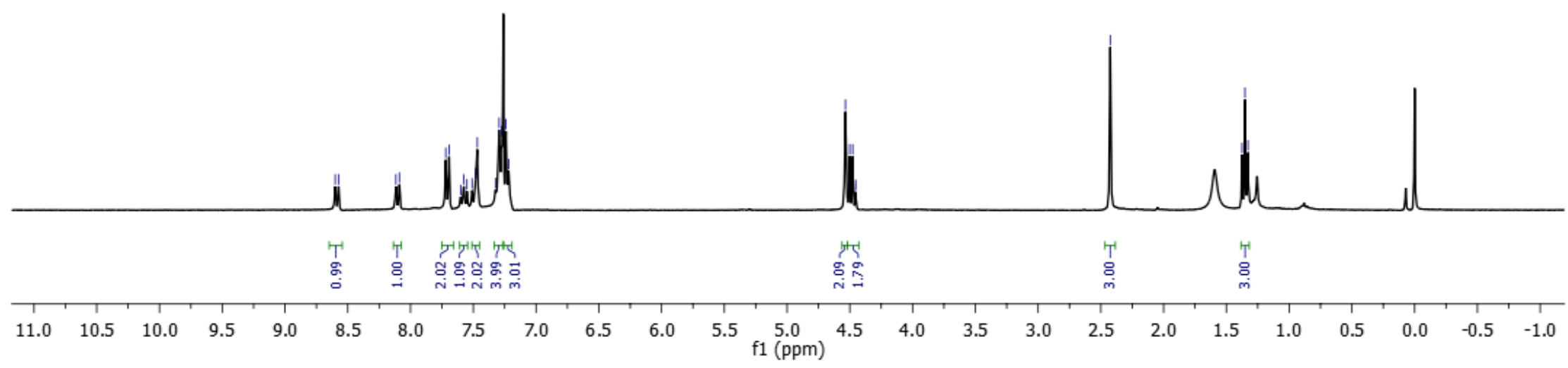




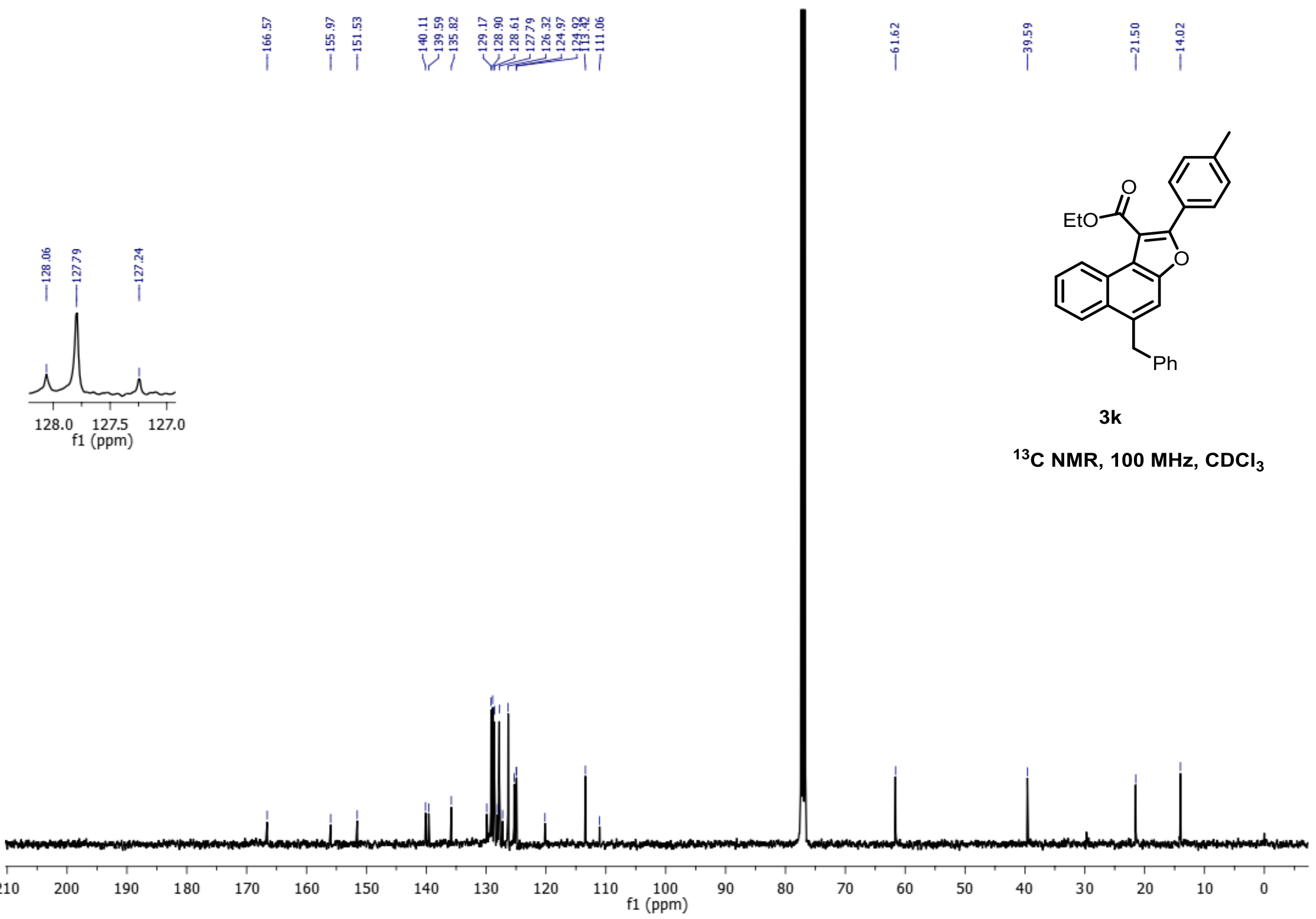




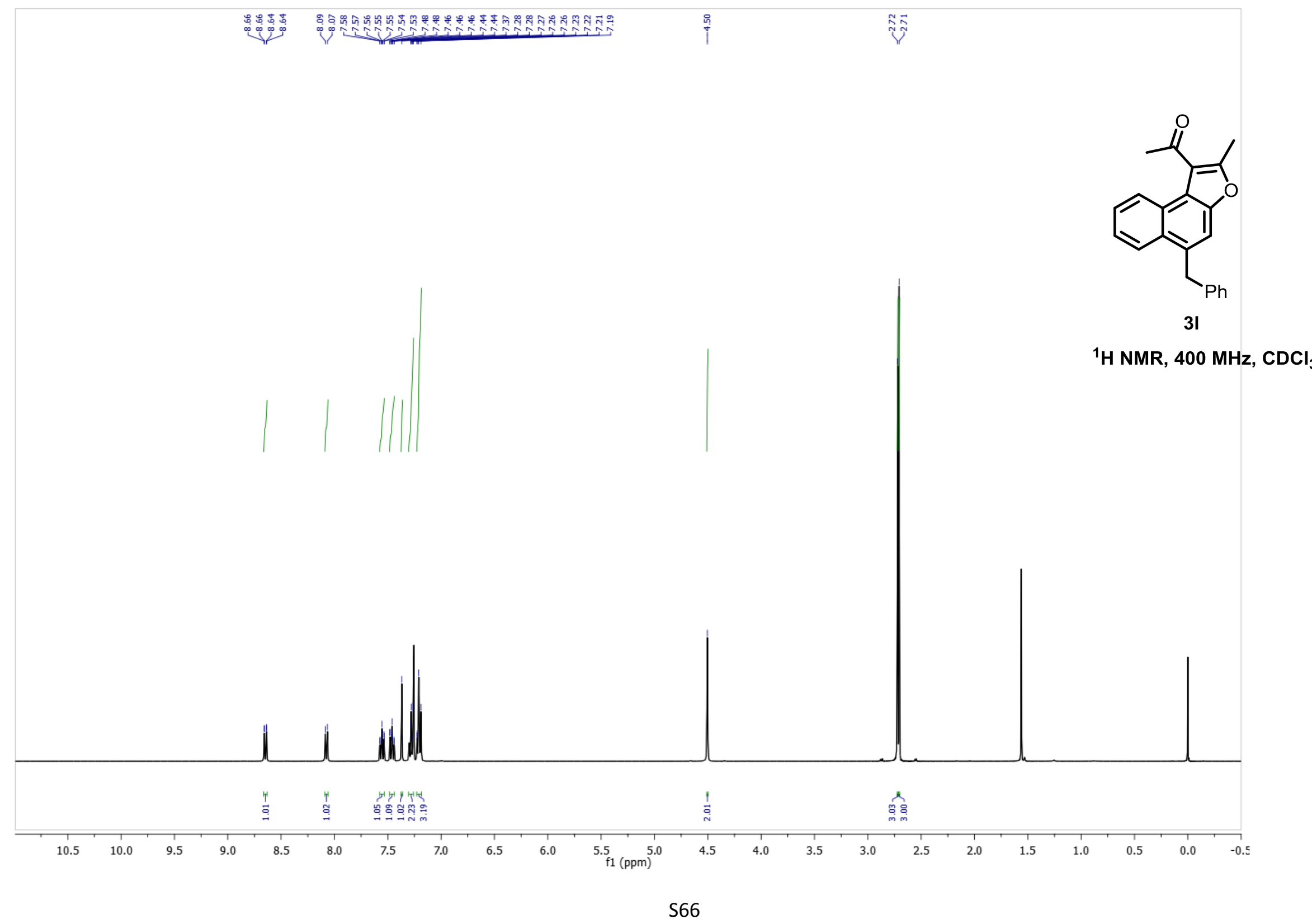




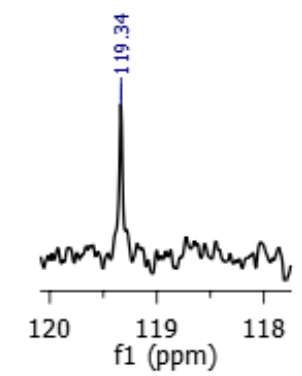

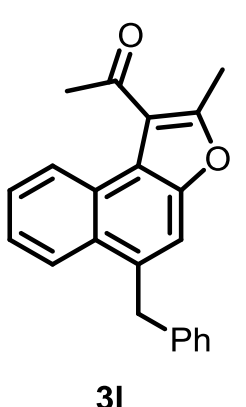

${ }^{13} \mathrm{C}$ NMR, $100 \mathrm{MHz}, \mathrm{CDCl}_{3}$

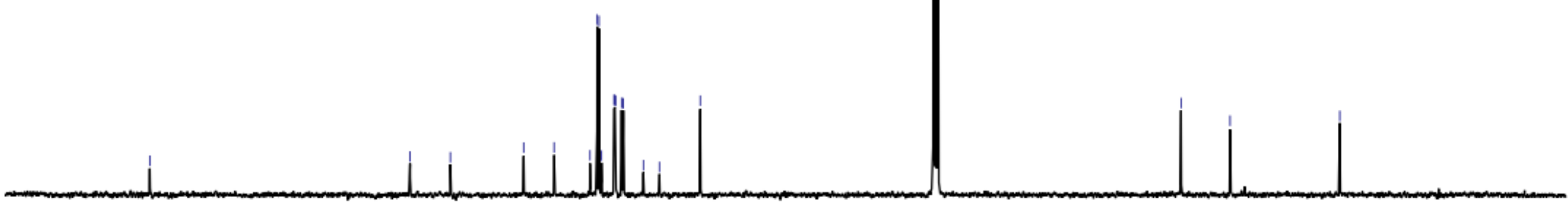

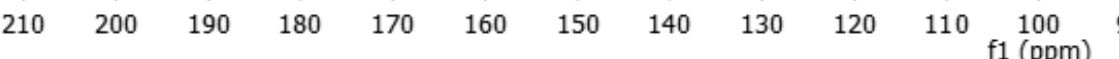




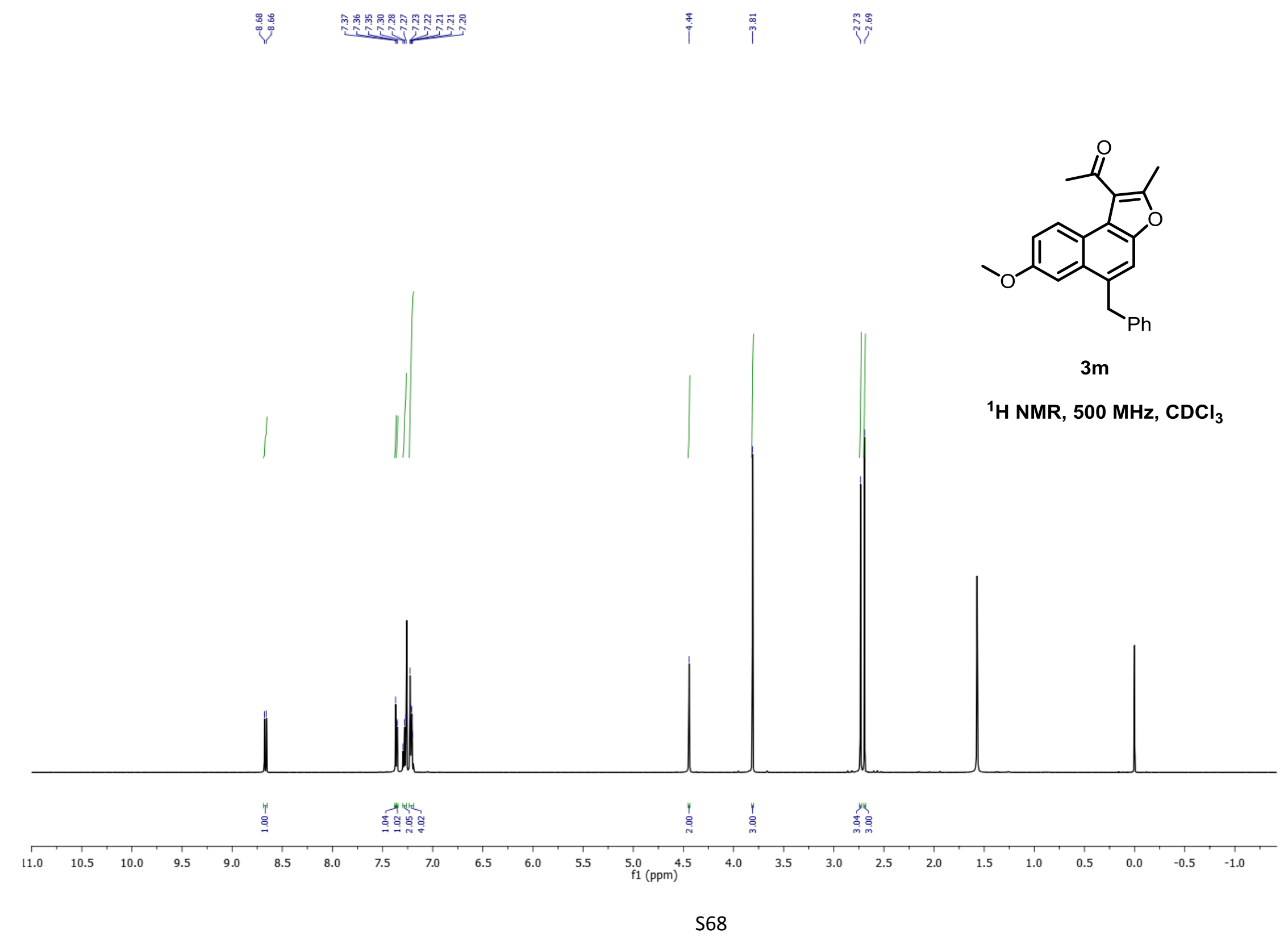



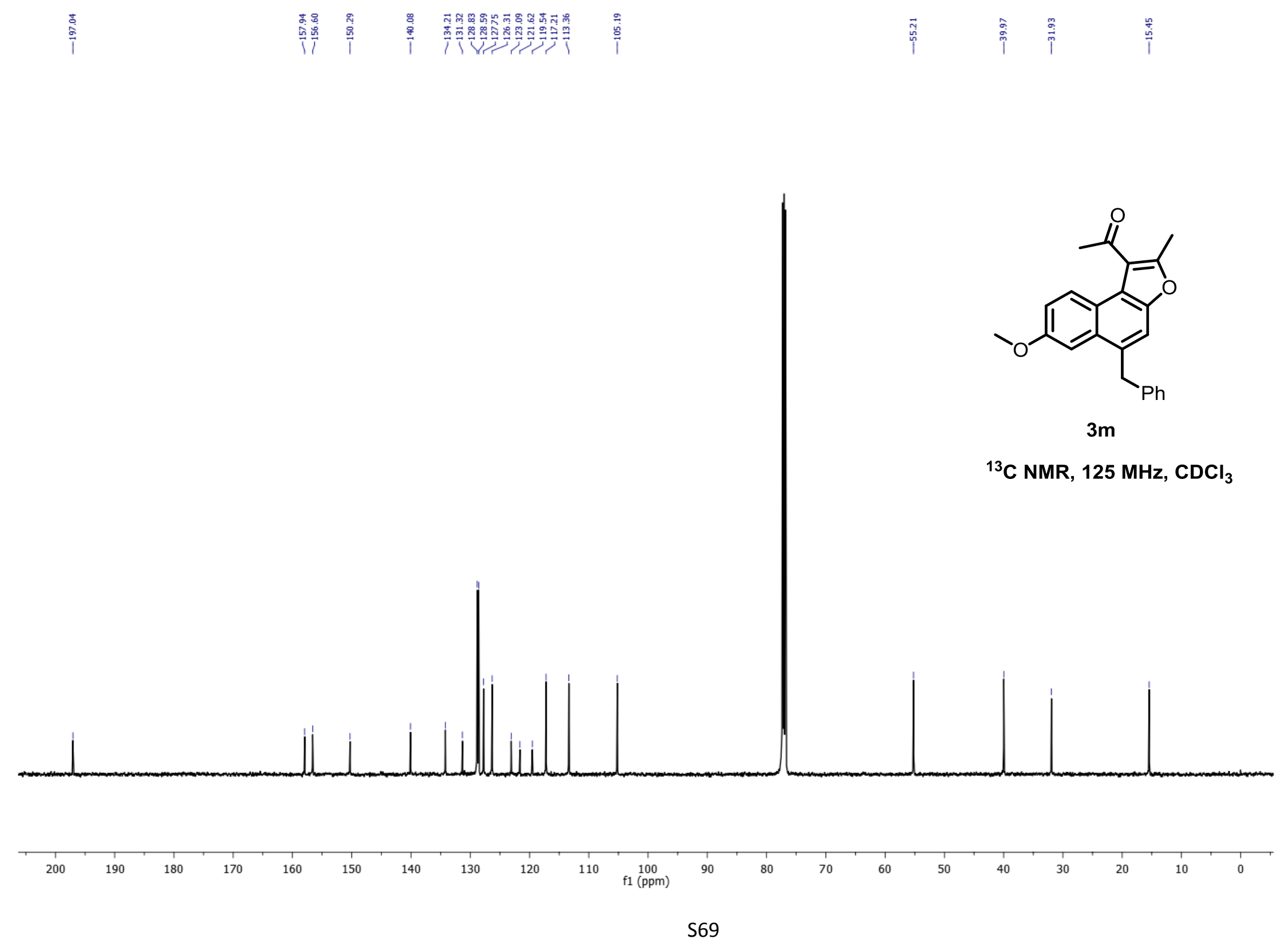


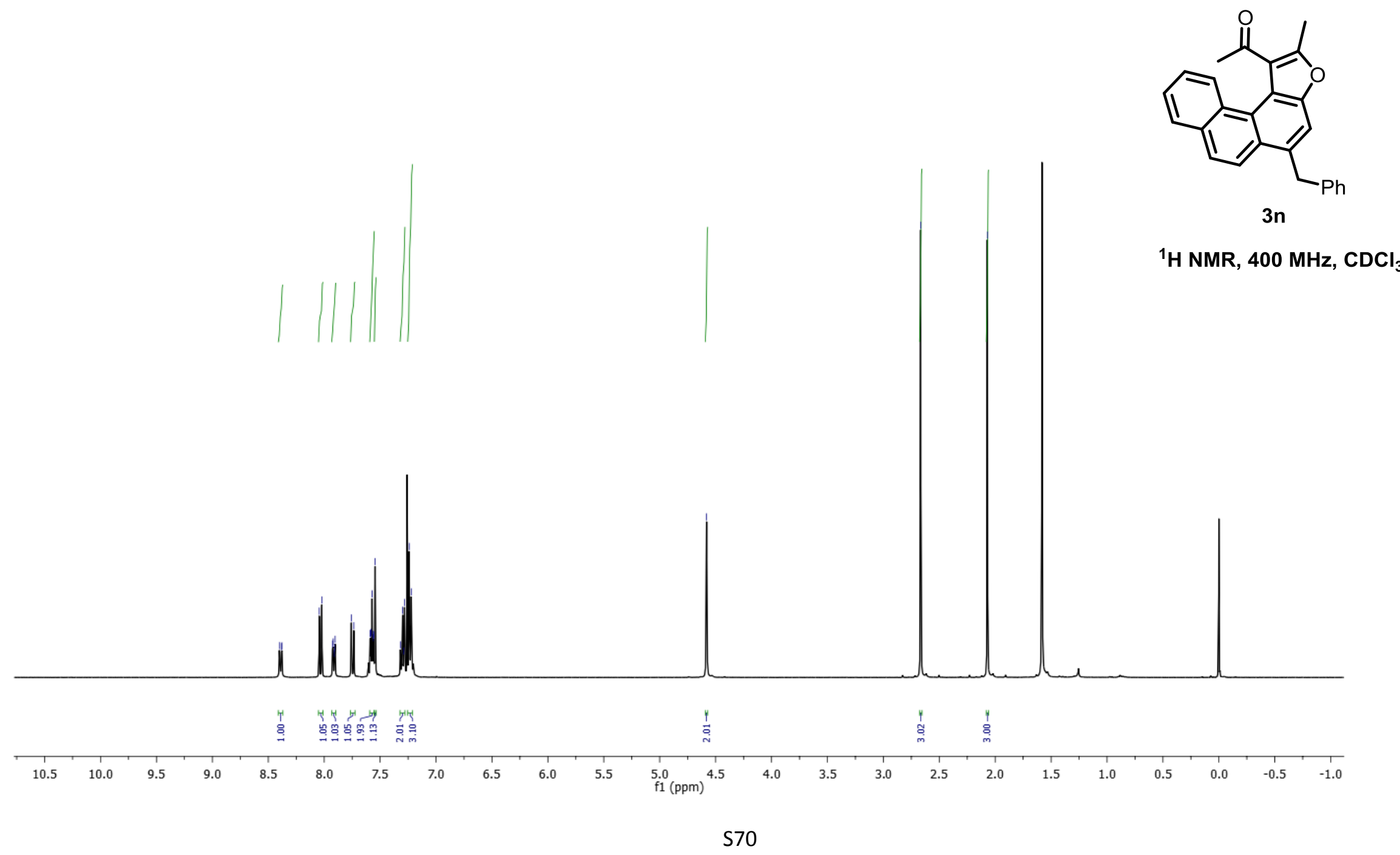



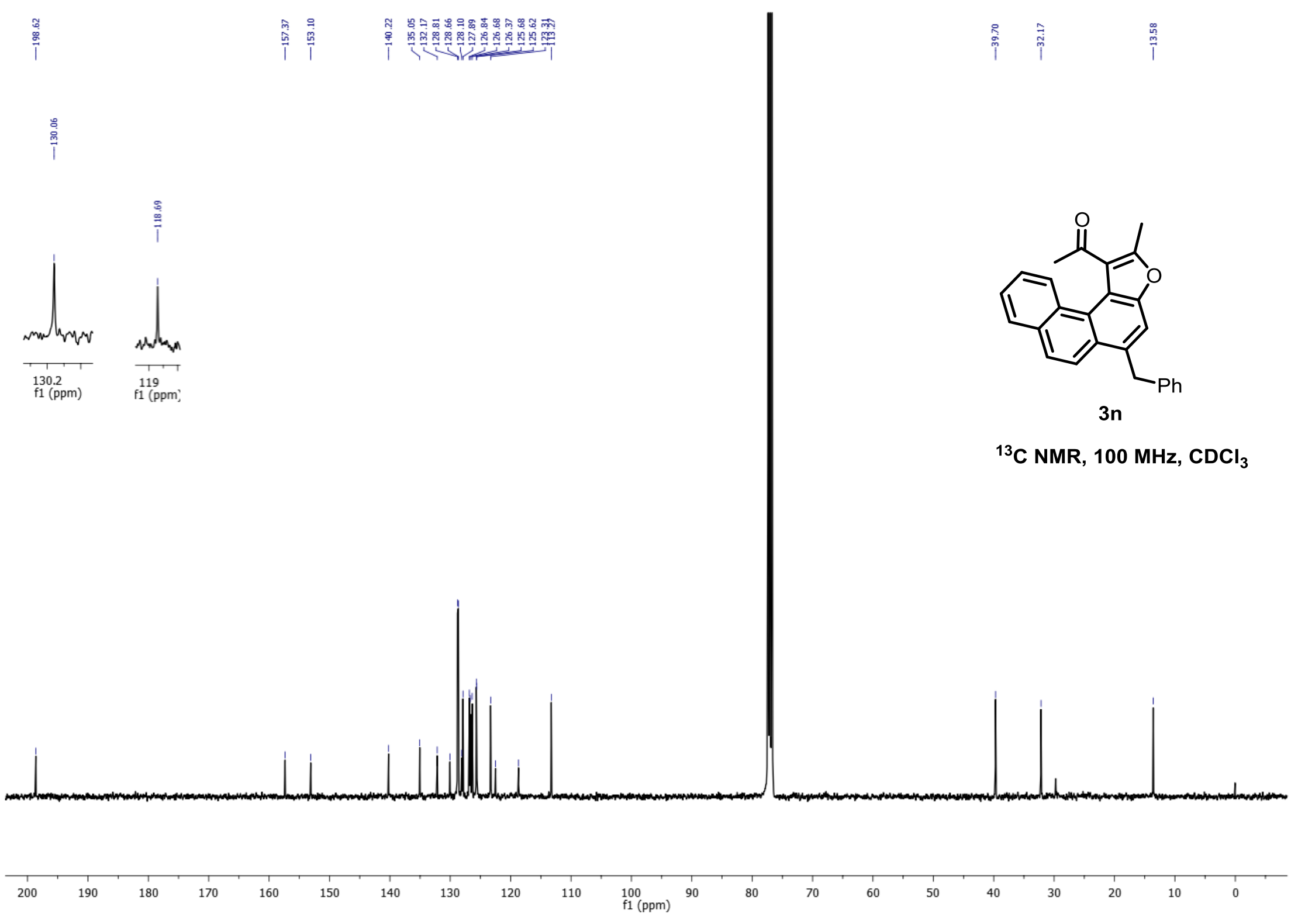


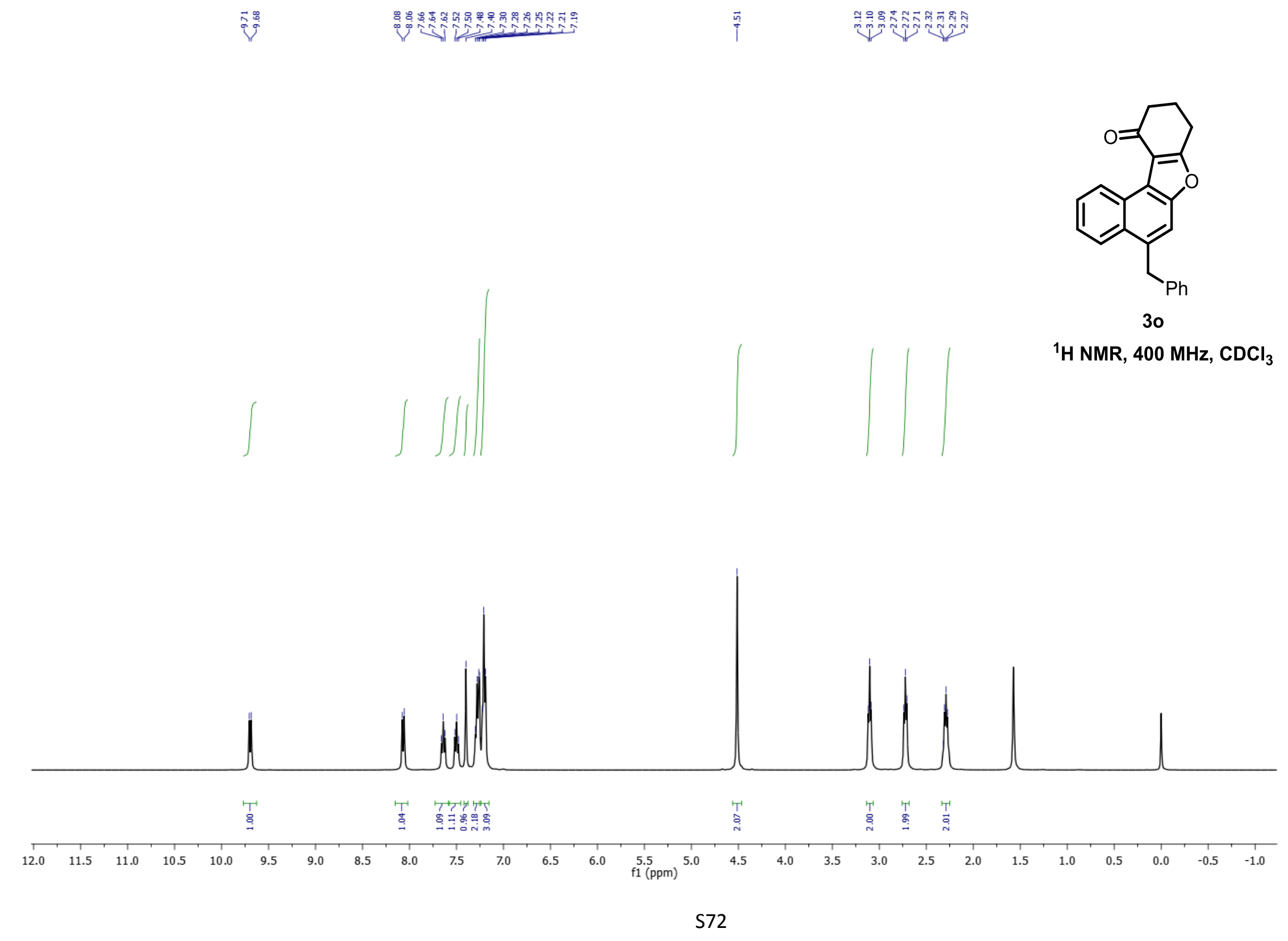



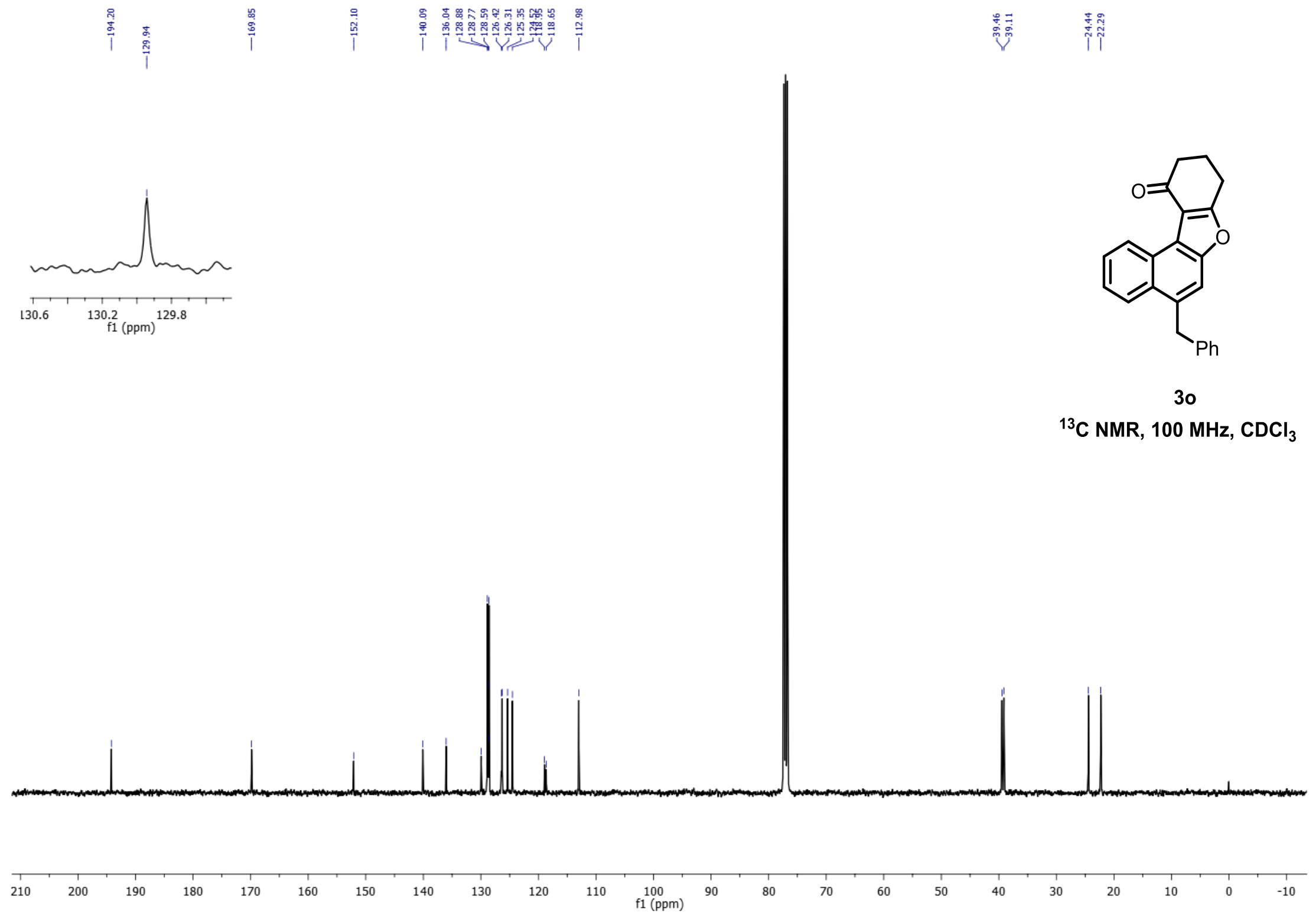


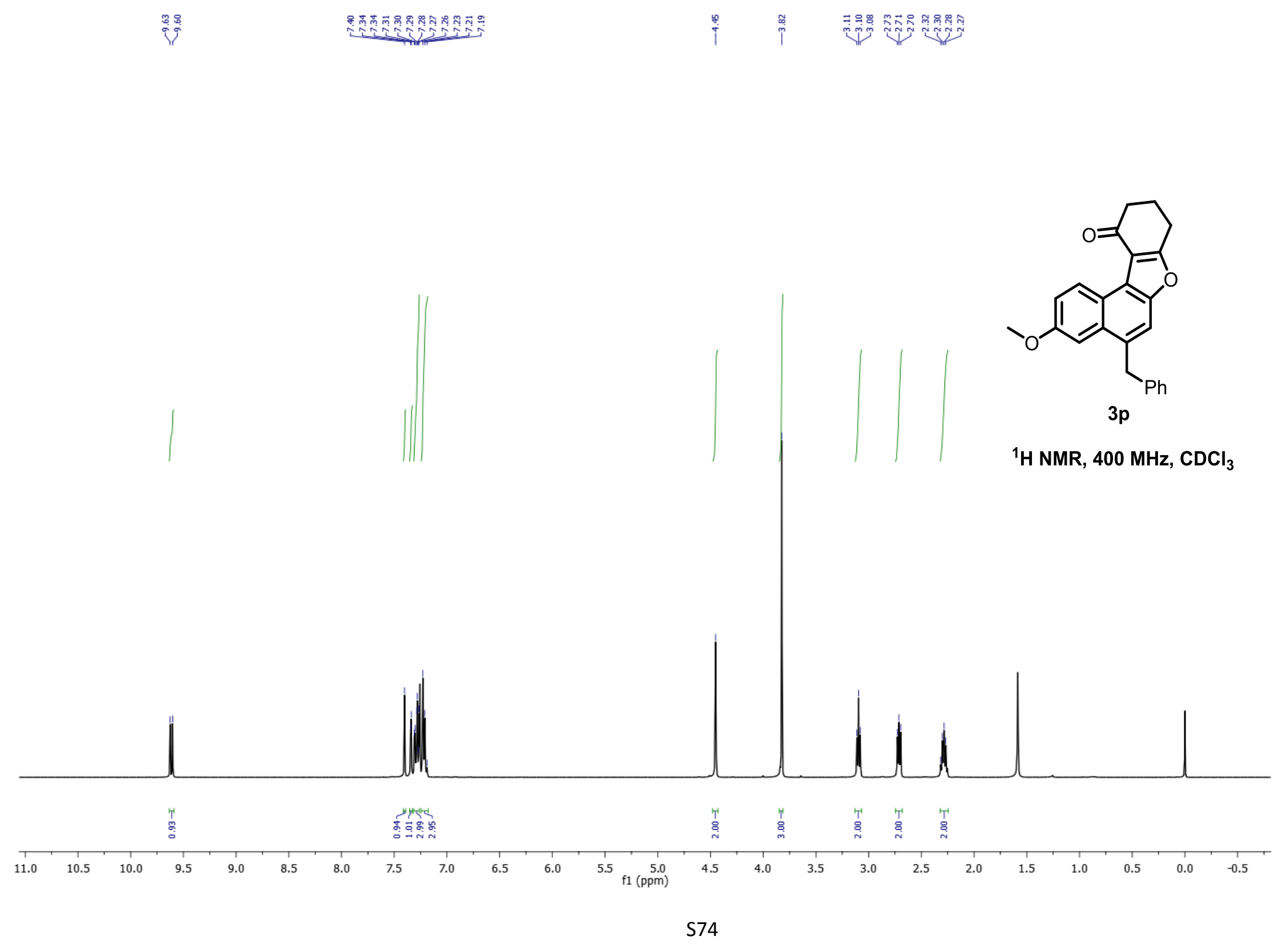




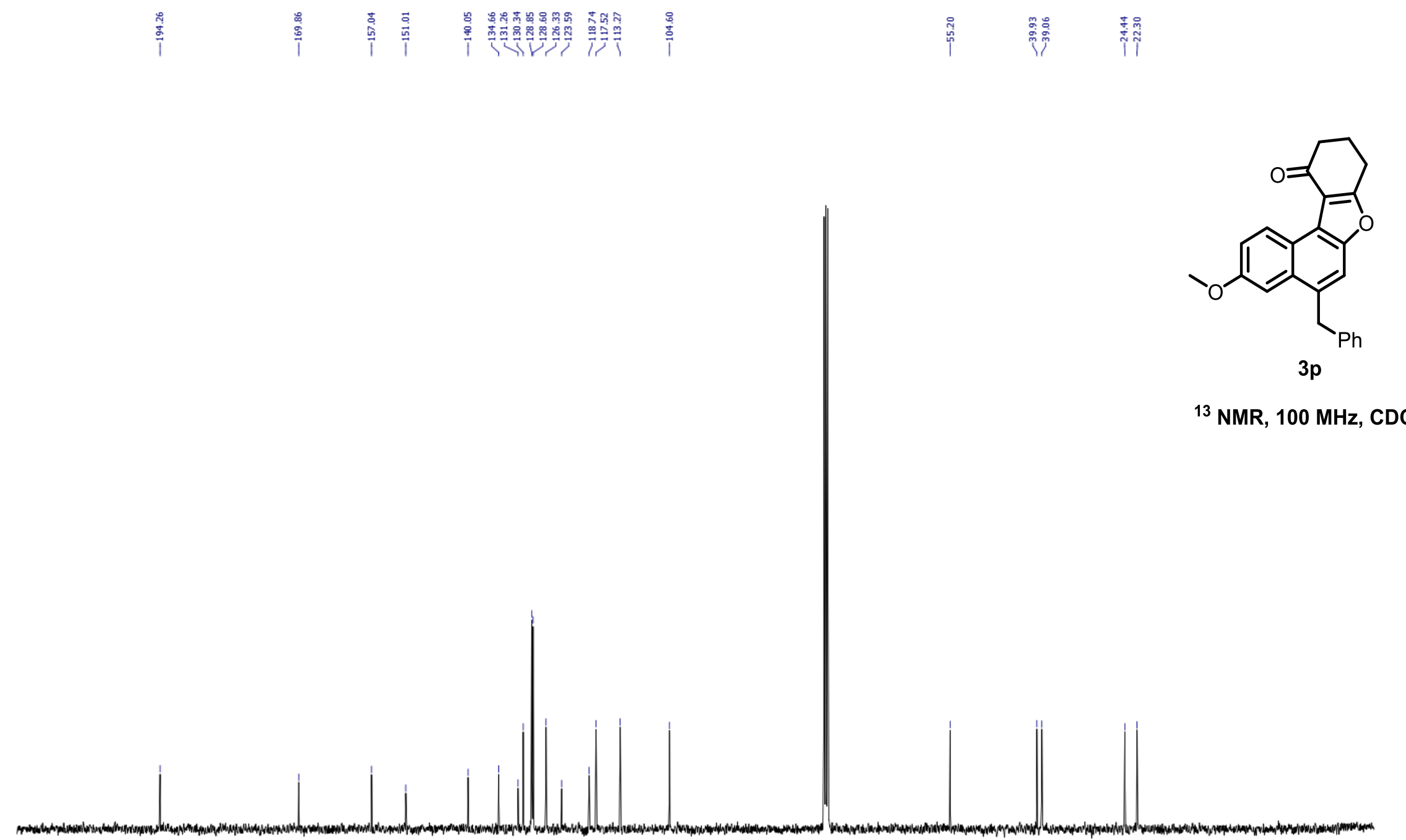




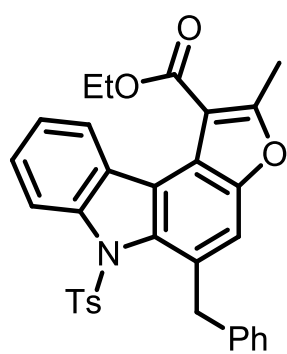

$3 q$

${ }^{1} \mathrm{H} \mathrm{NMR,}, 400 \mathrm{MHz}, \mathrm{CDCl}_{3}$

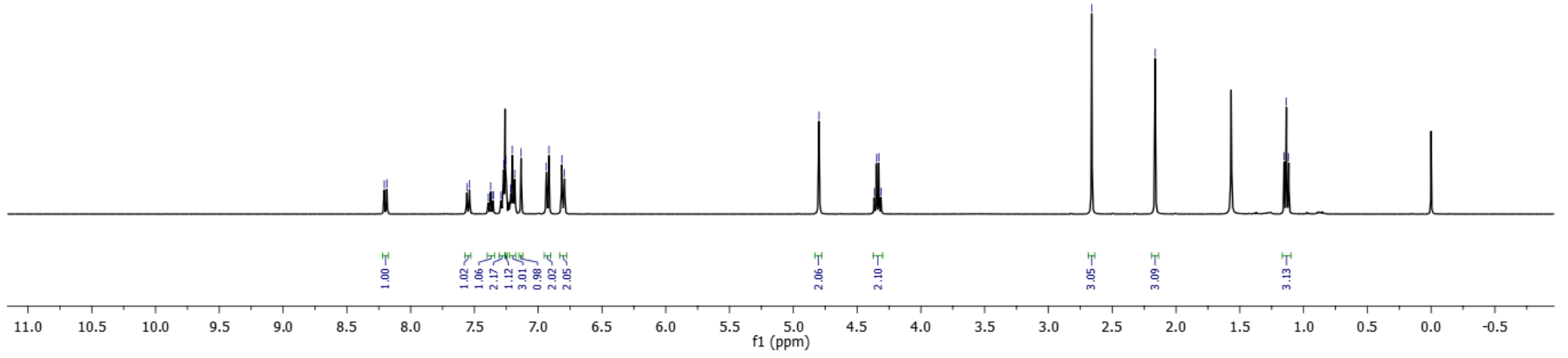




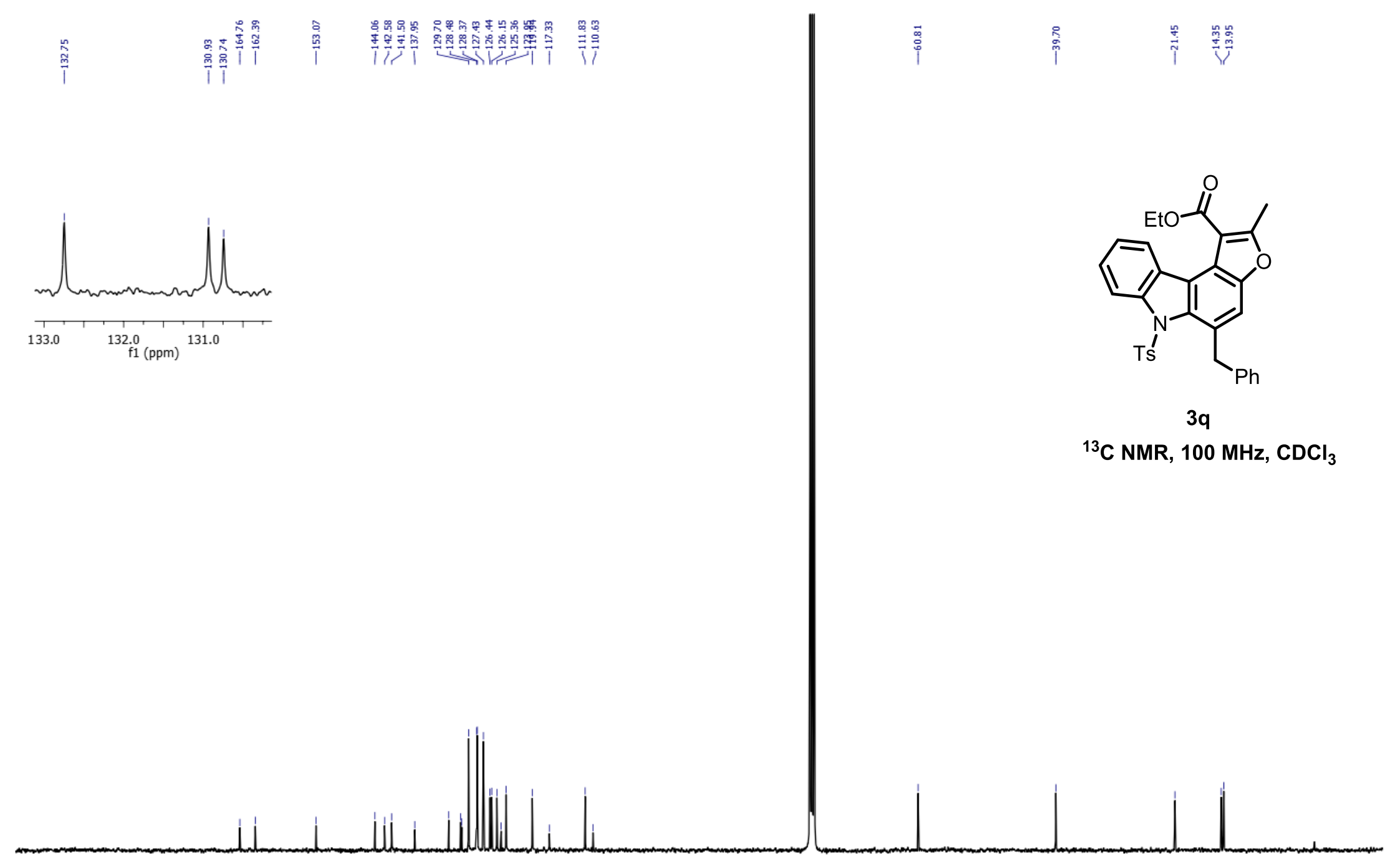

190
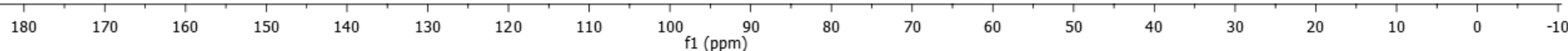


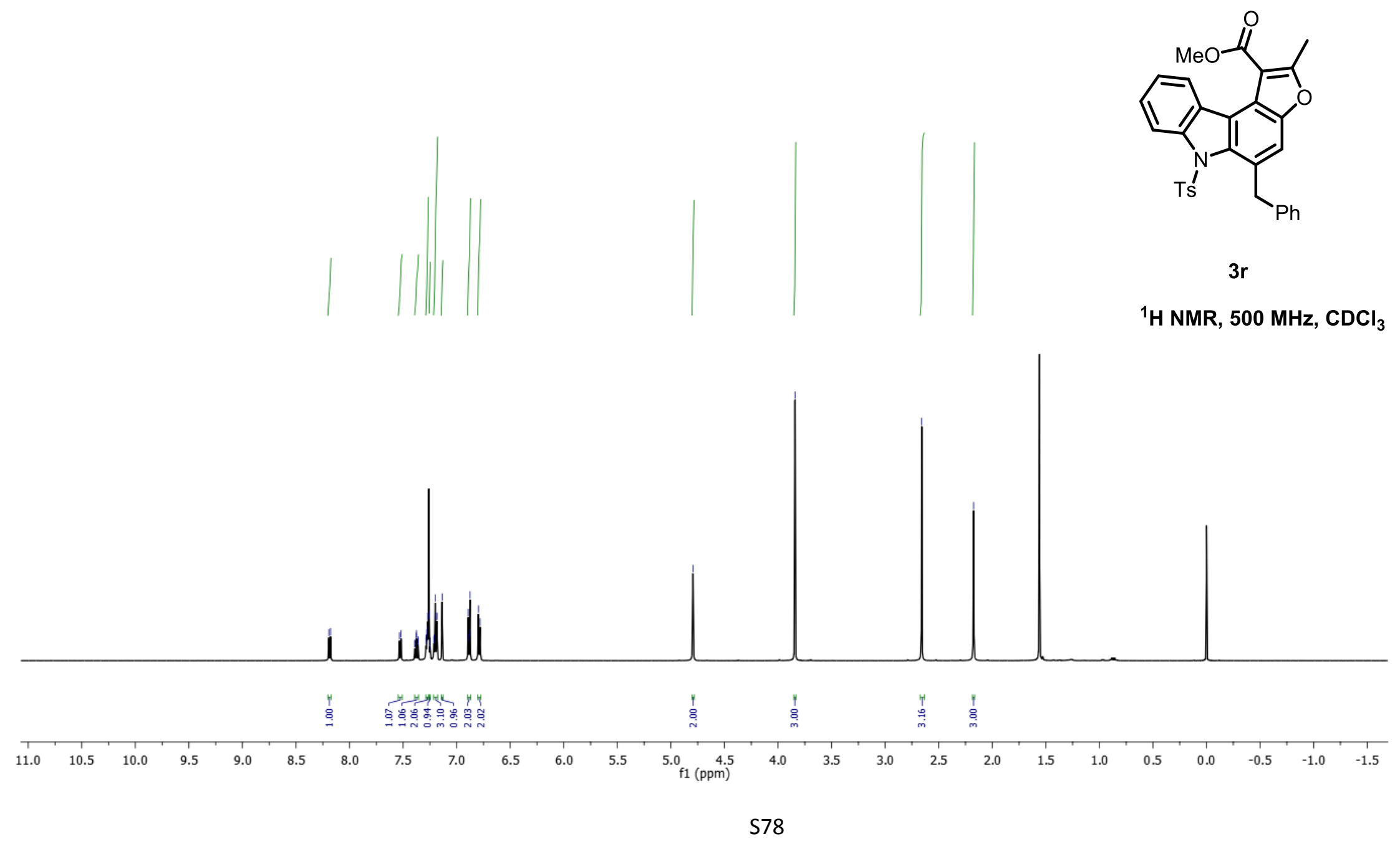




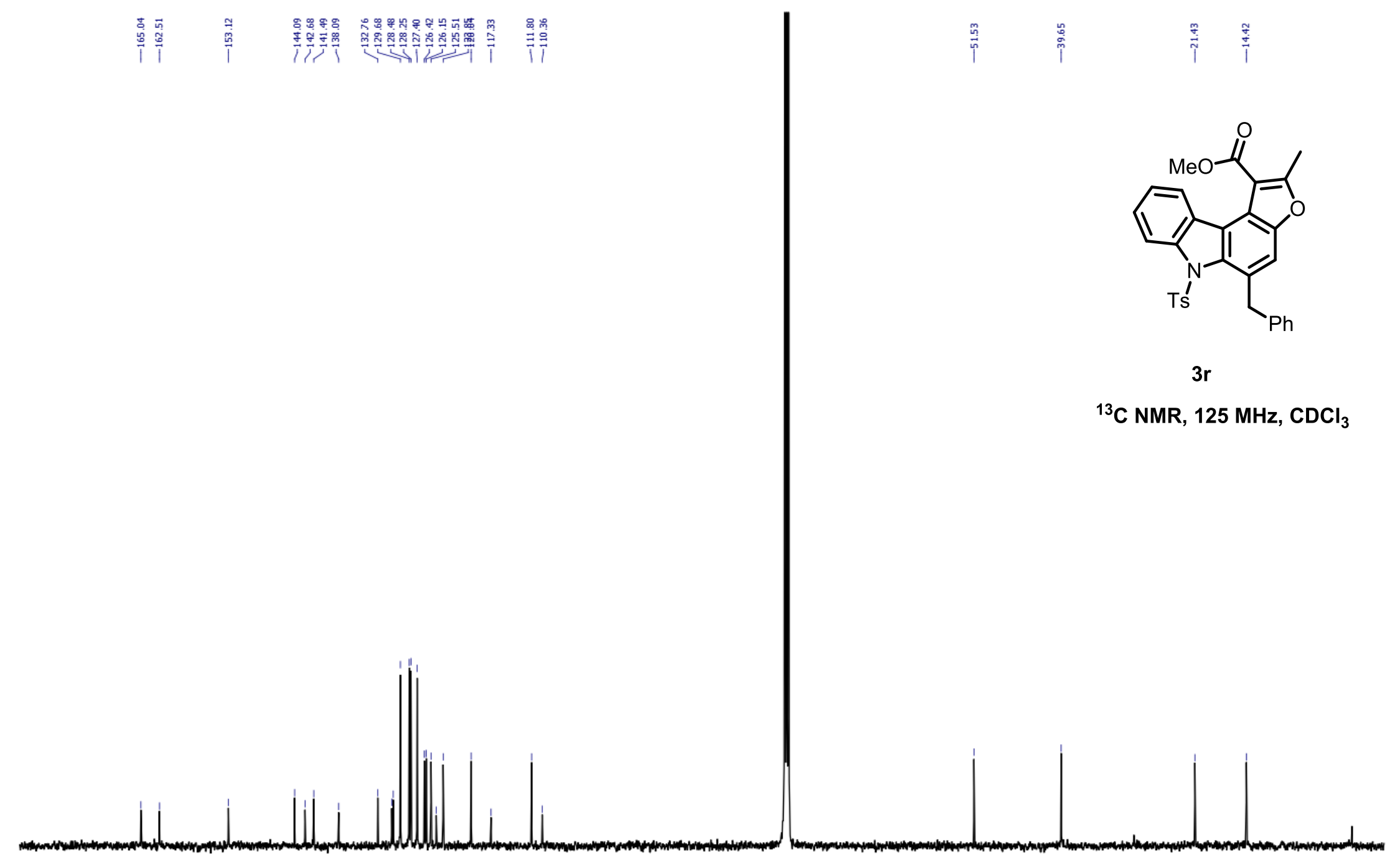

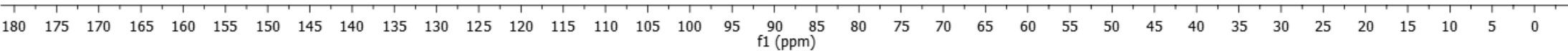




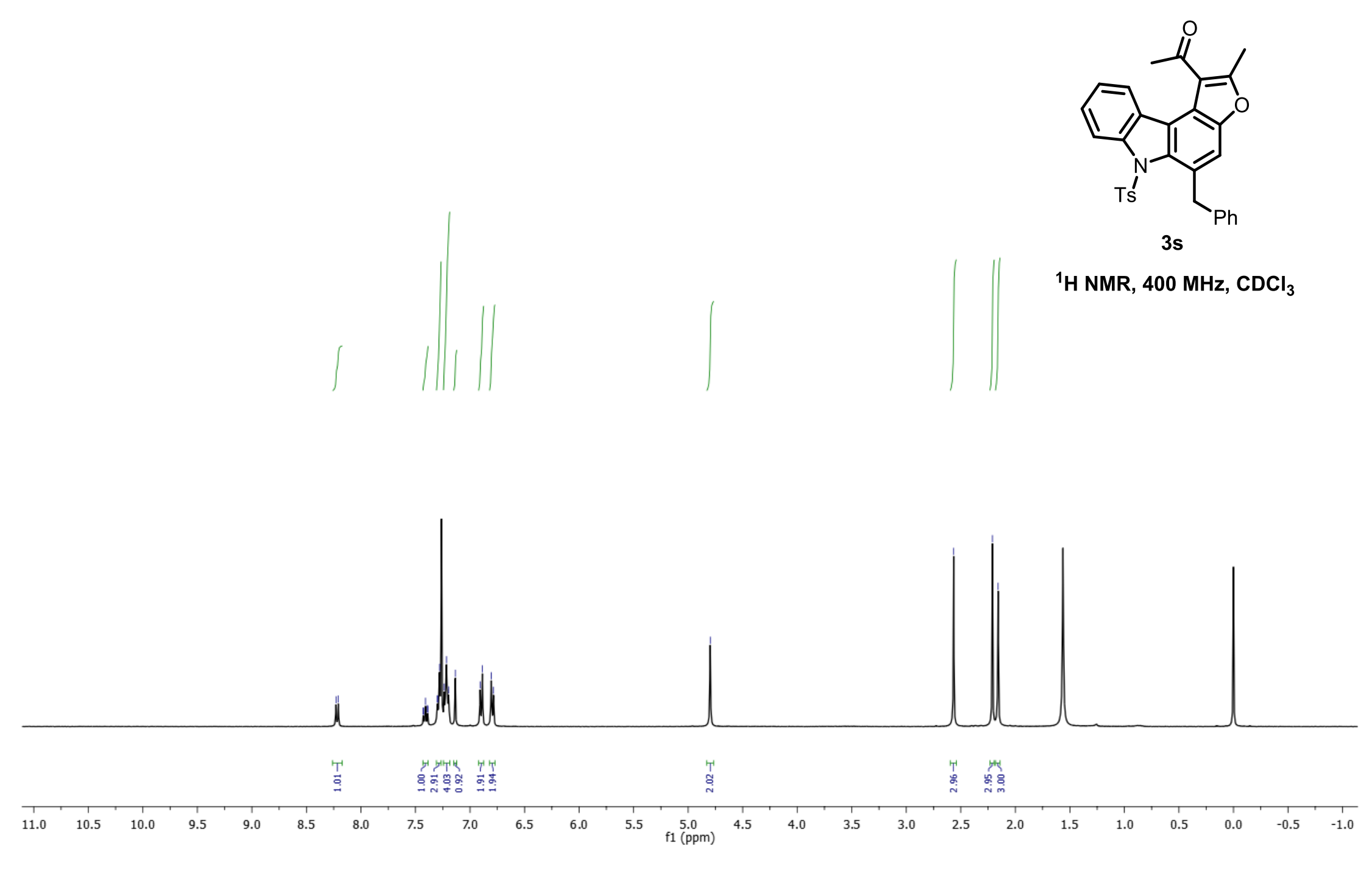



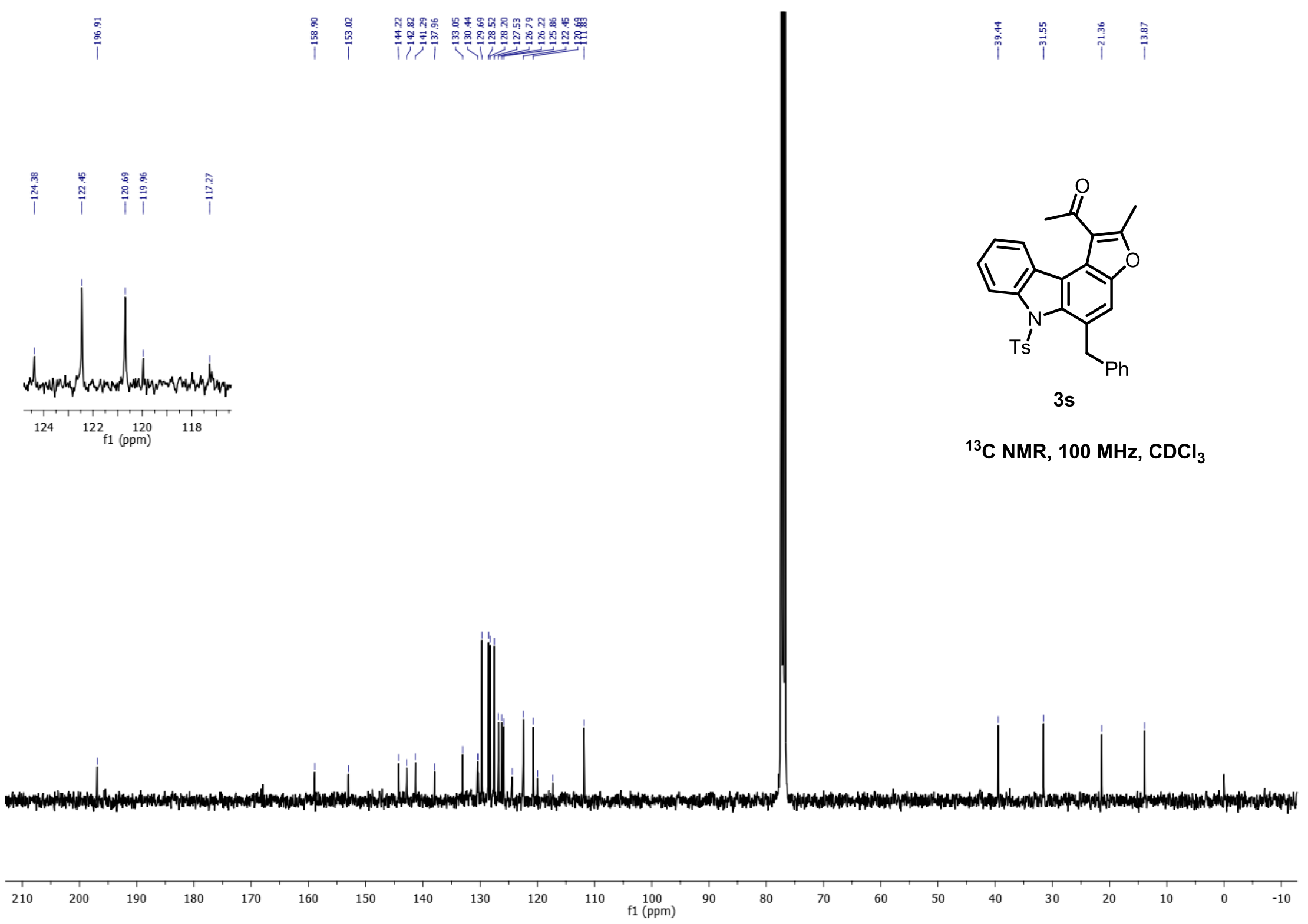


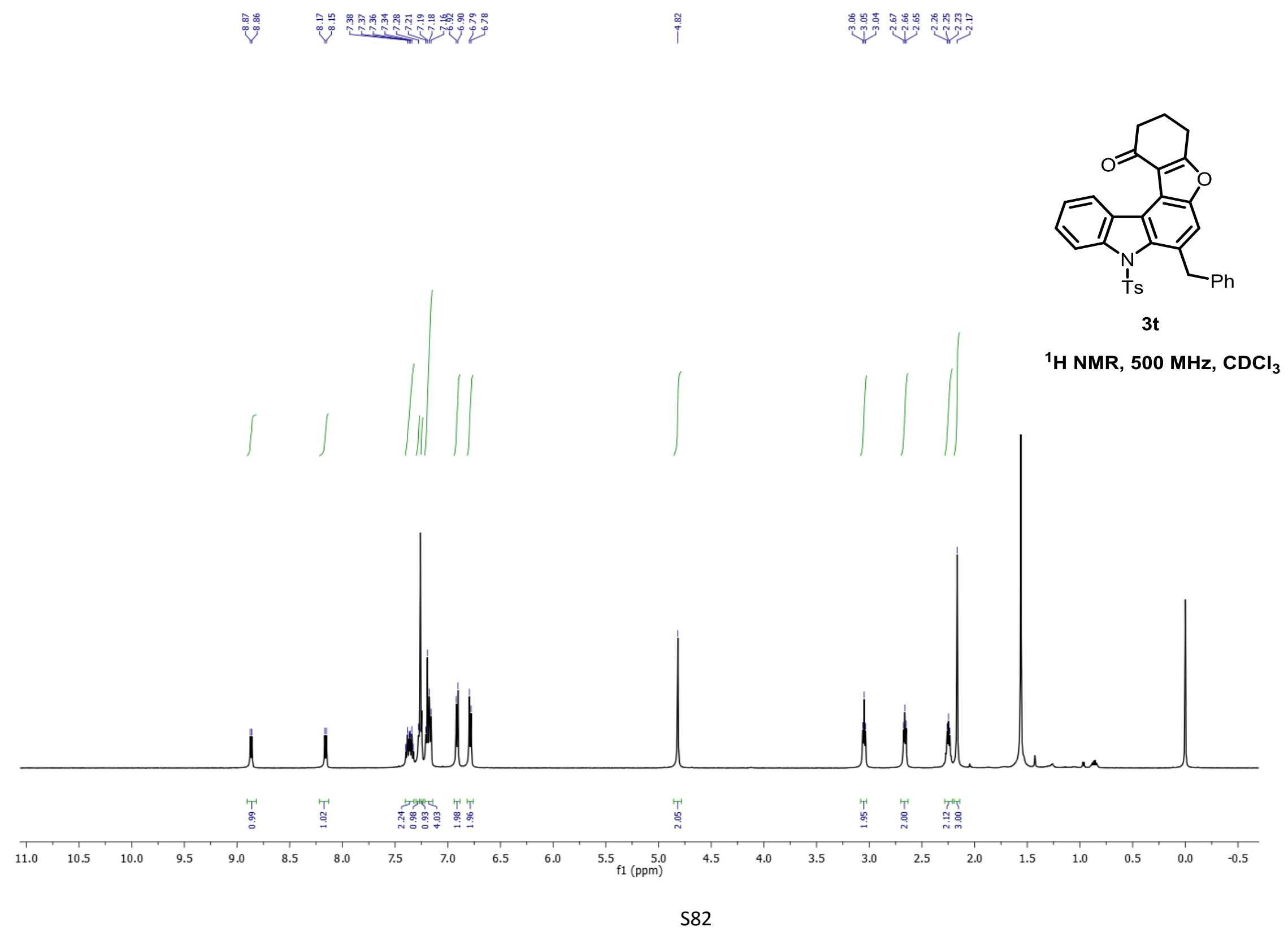



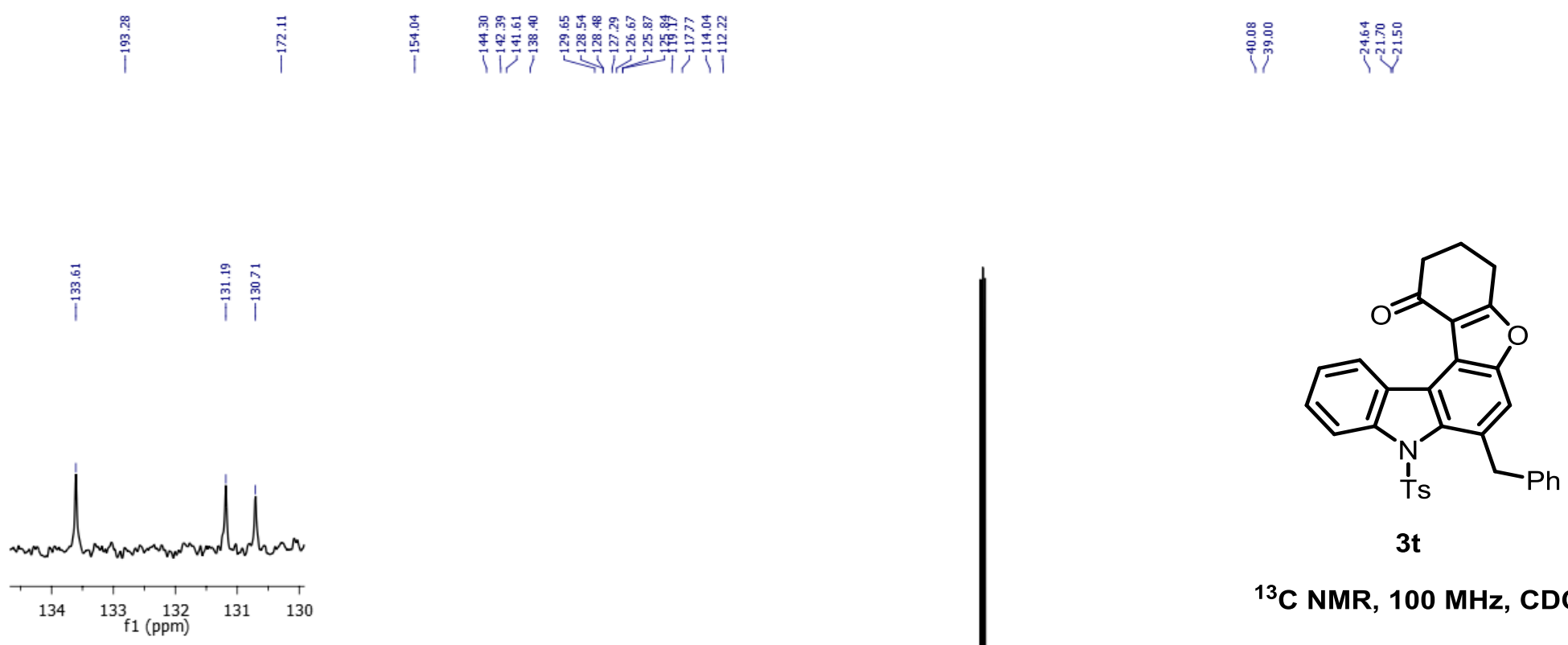

${ }^{13} \mathrm{C}$ NMR, $100 \mathrm{MHz} \mathrm{CDCl}_{3}$

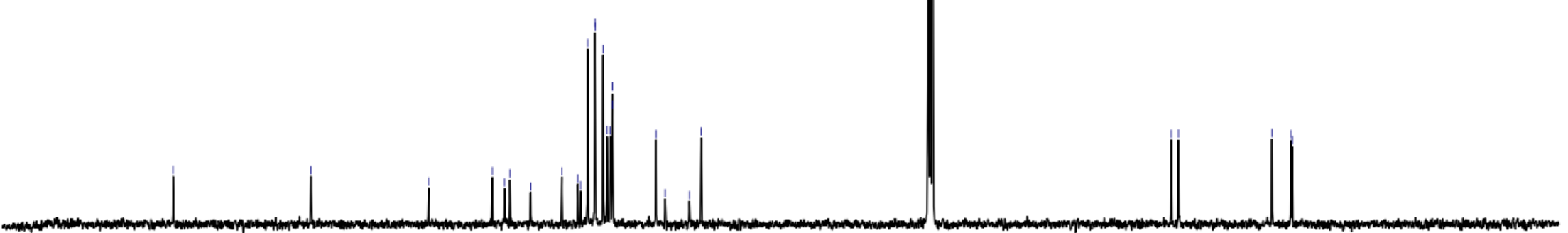



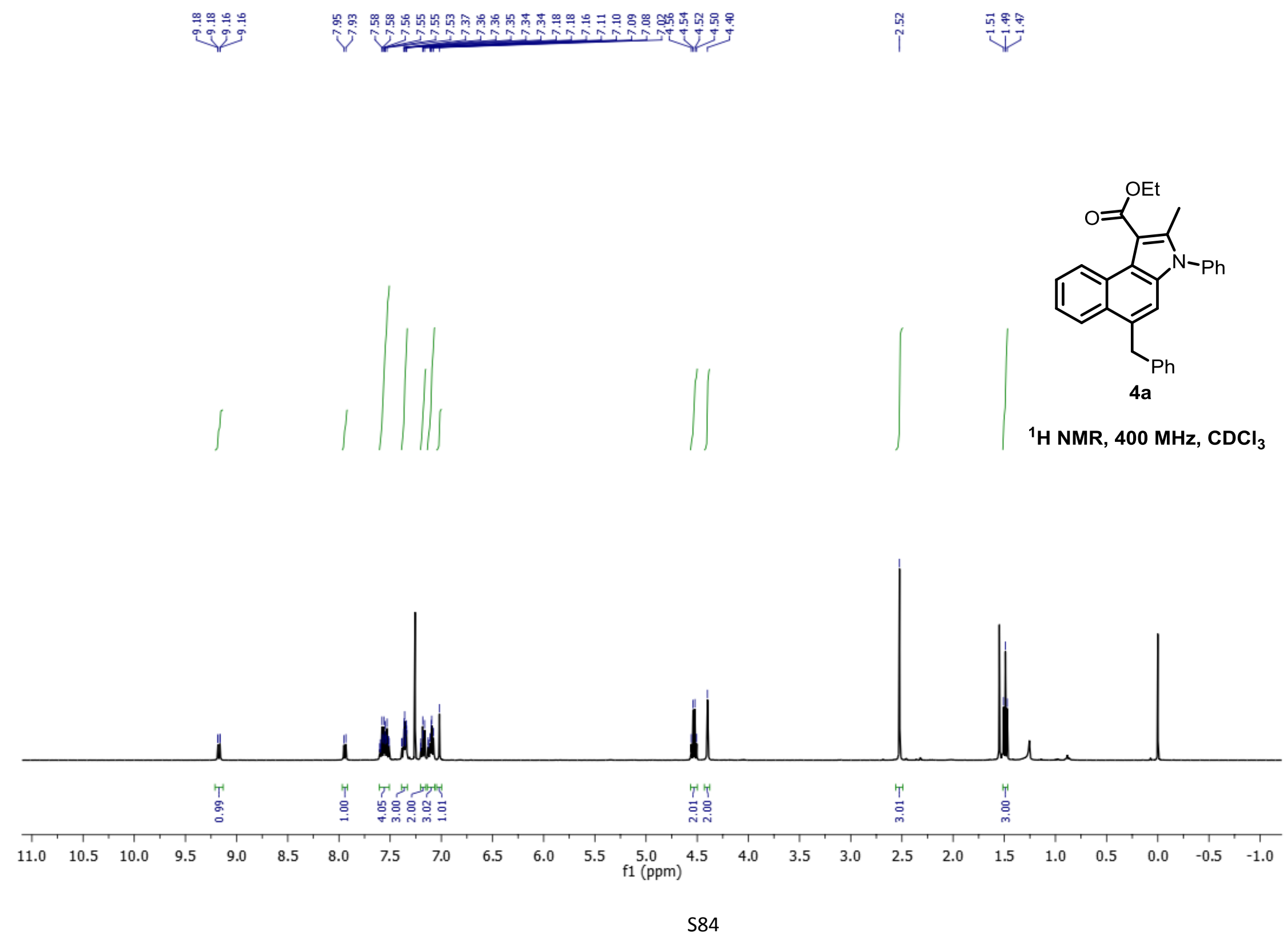


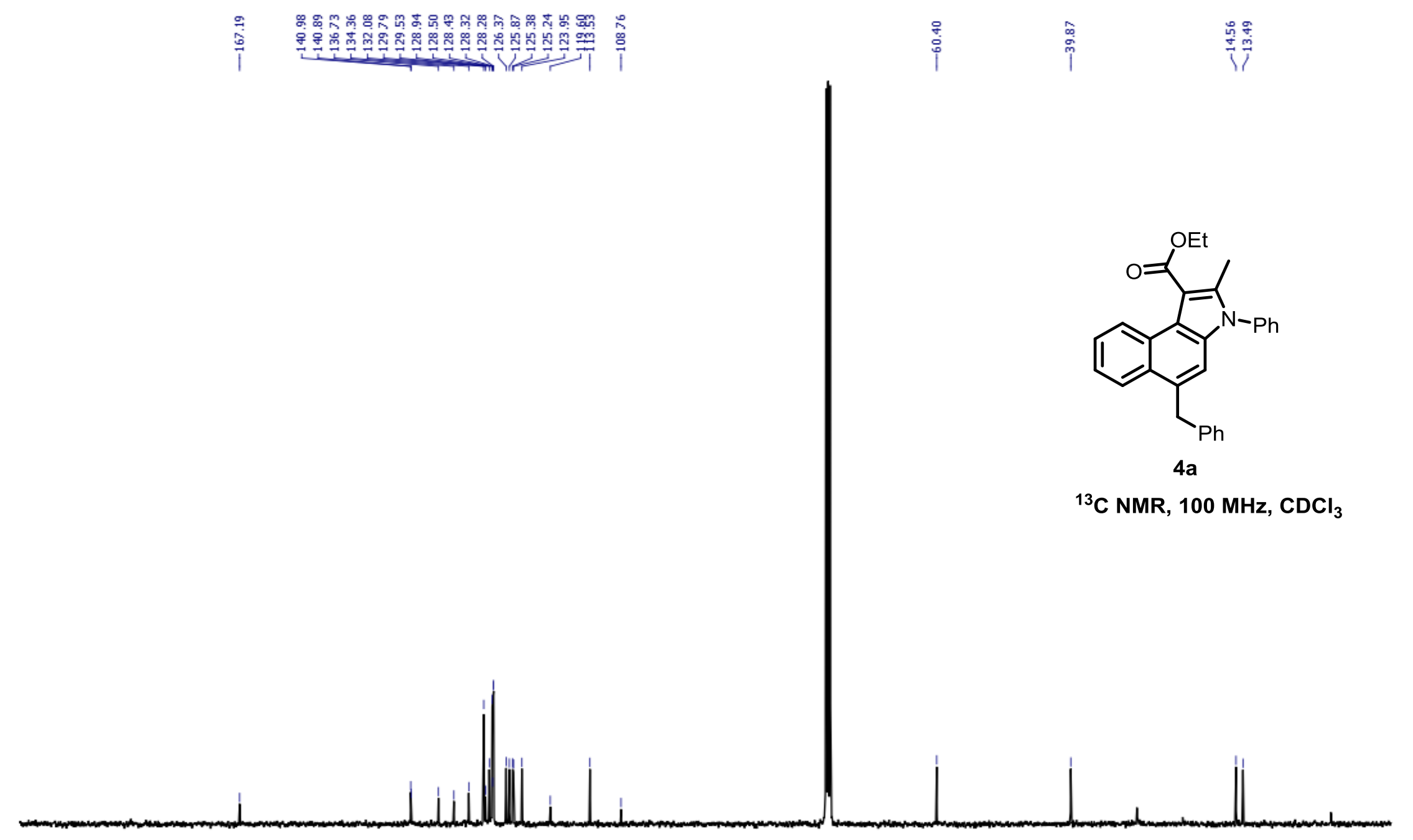

$\begin{array}{lllllllllll}200 & 190 & 180 & 170 & 160 & 150 & 140 & 130 & 120 & 110 & \begin{array}{c}100 \\ \mathrm{f} 1(\mathrm{ppm})\end{array}\end{array}$ 


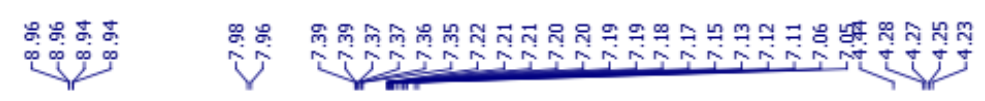

ขึ

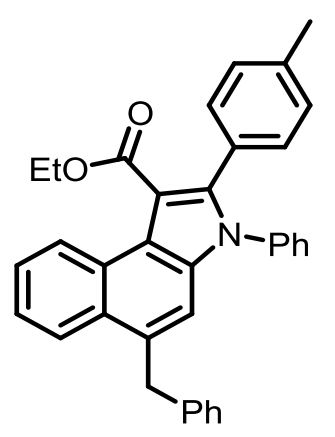

$4 b$

${ }^{1} \mathrm{H}$ NMR, $400 \mathrm{MHz} \mathrm{CDCl}_{3}$

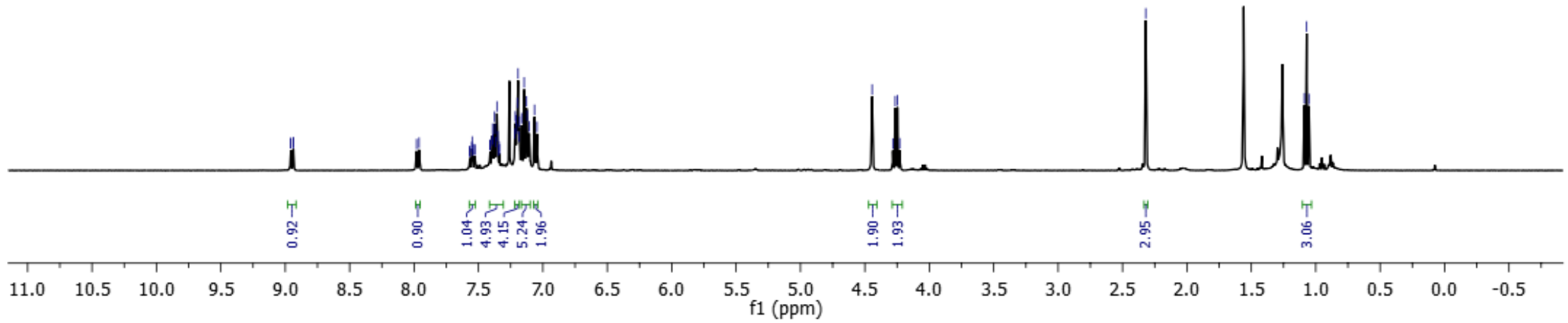




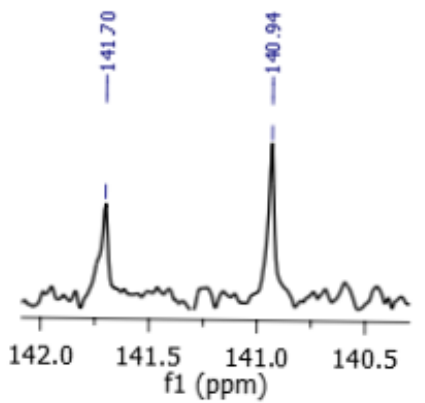

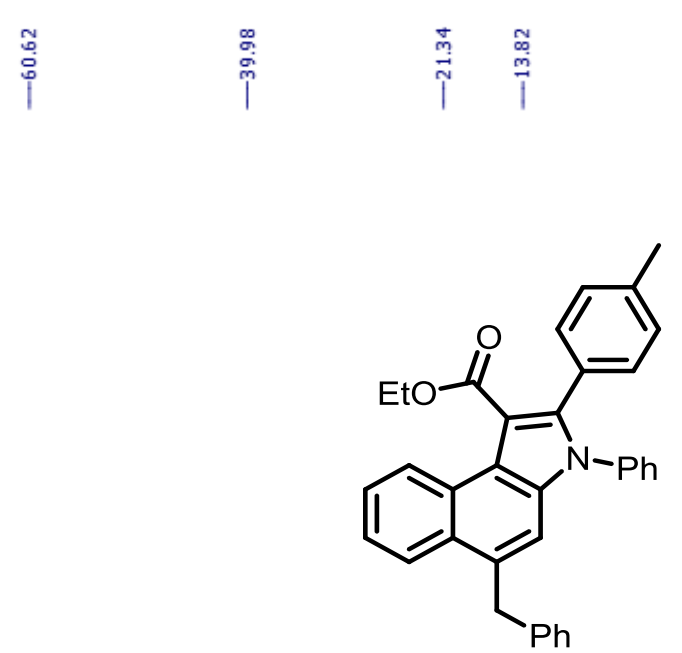

4b

${ }^{13} \mathrm{C}$ NMR, $125 \mathrm{MHz}, \mathrm{CDCl}_{3}$

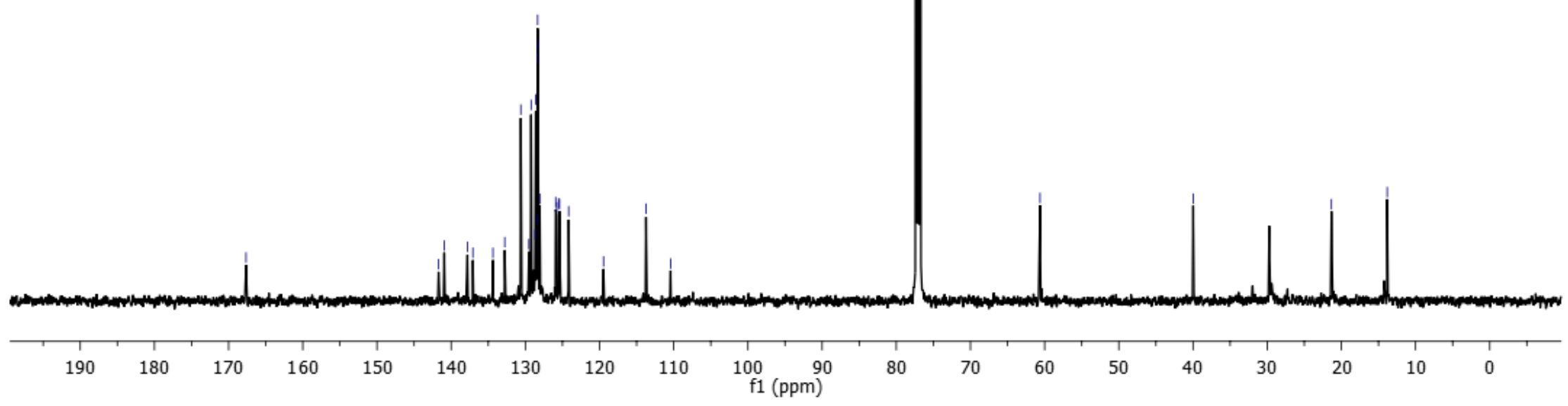




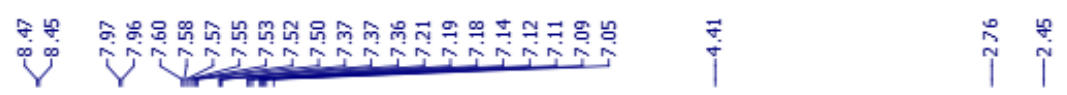

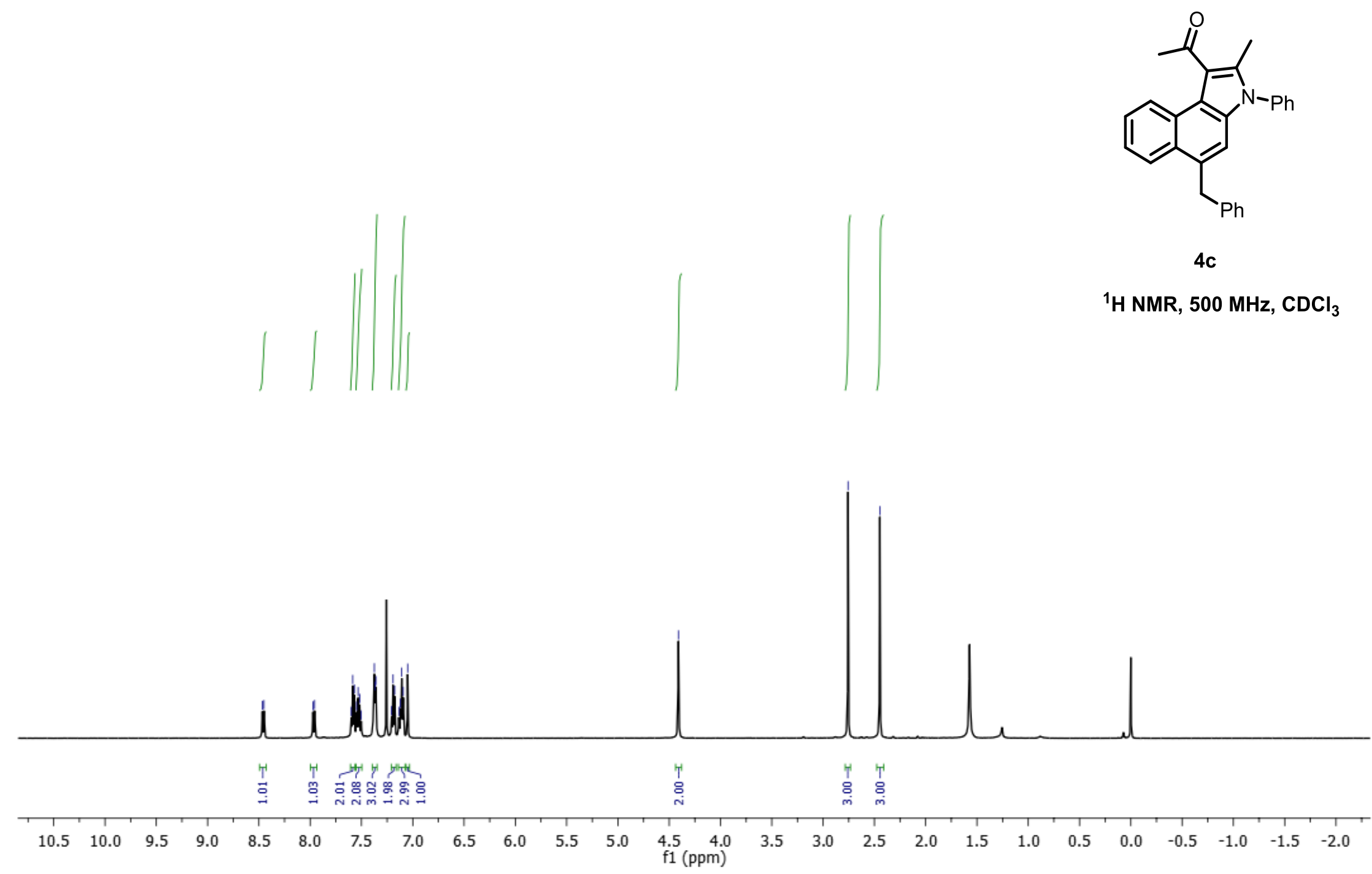




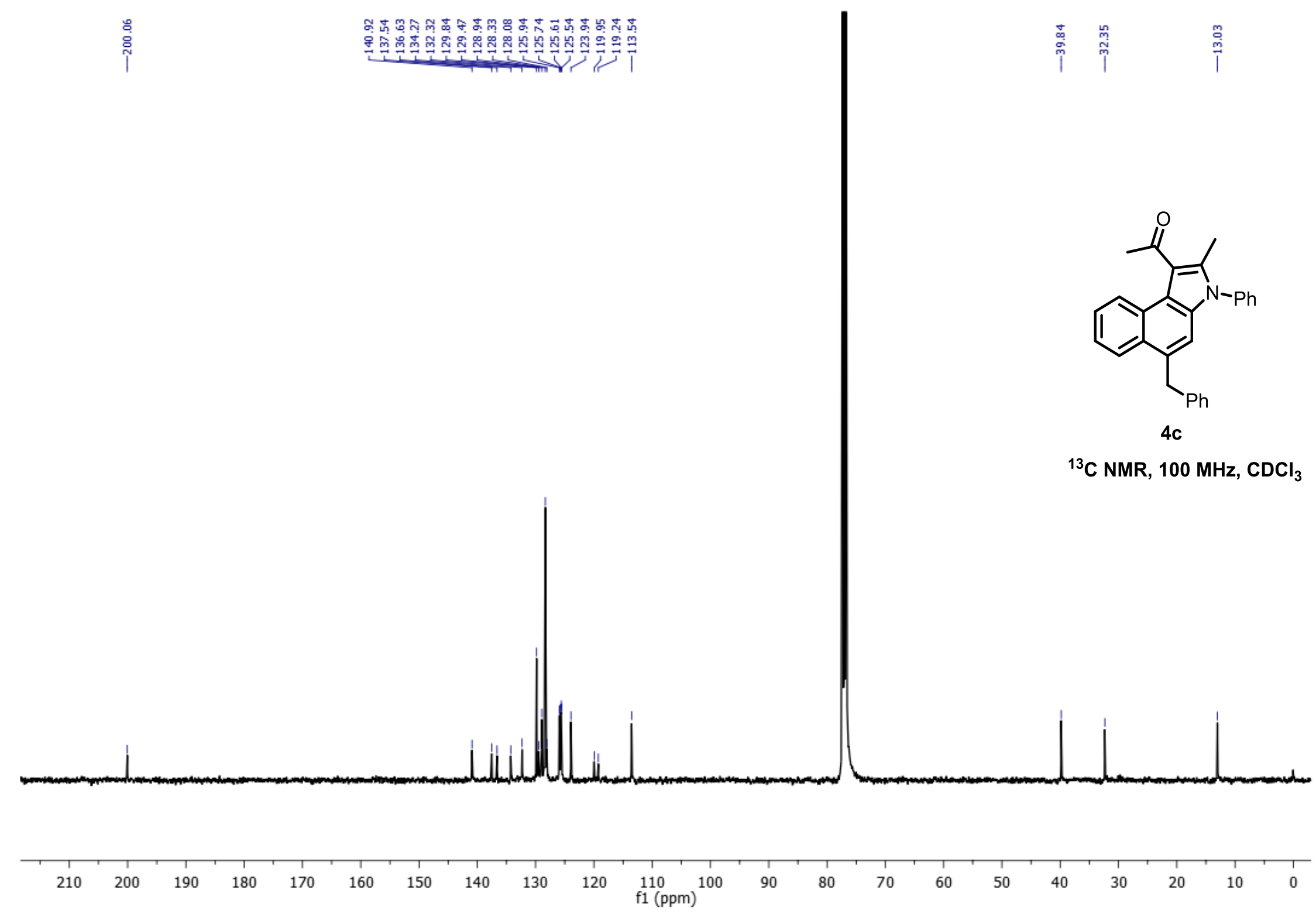




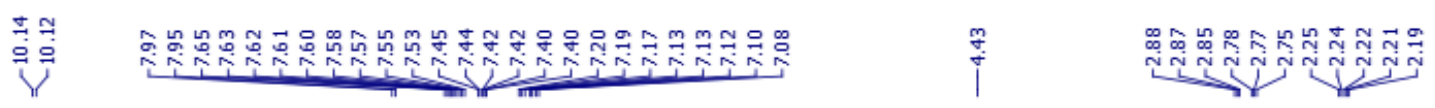

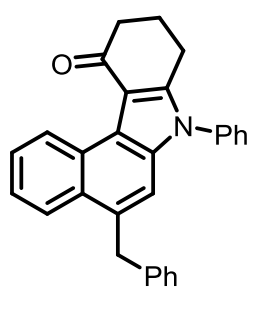

4d

${ }^{1} \mathrm{H} \mathrm{NMR}, 400 \mathrm{MHz}, \mathrm{CDCl}_{3}$
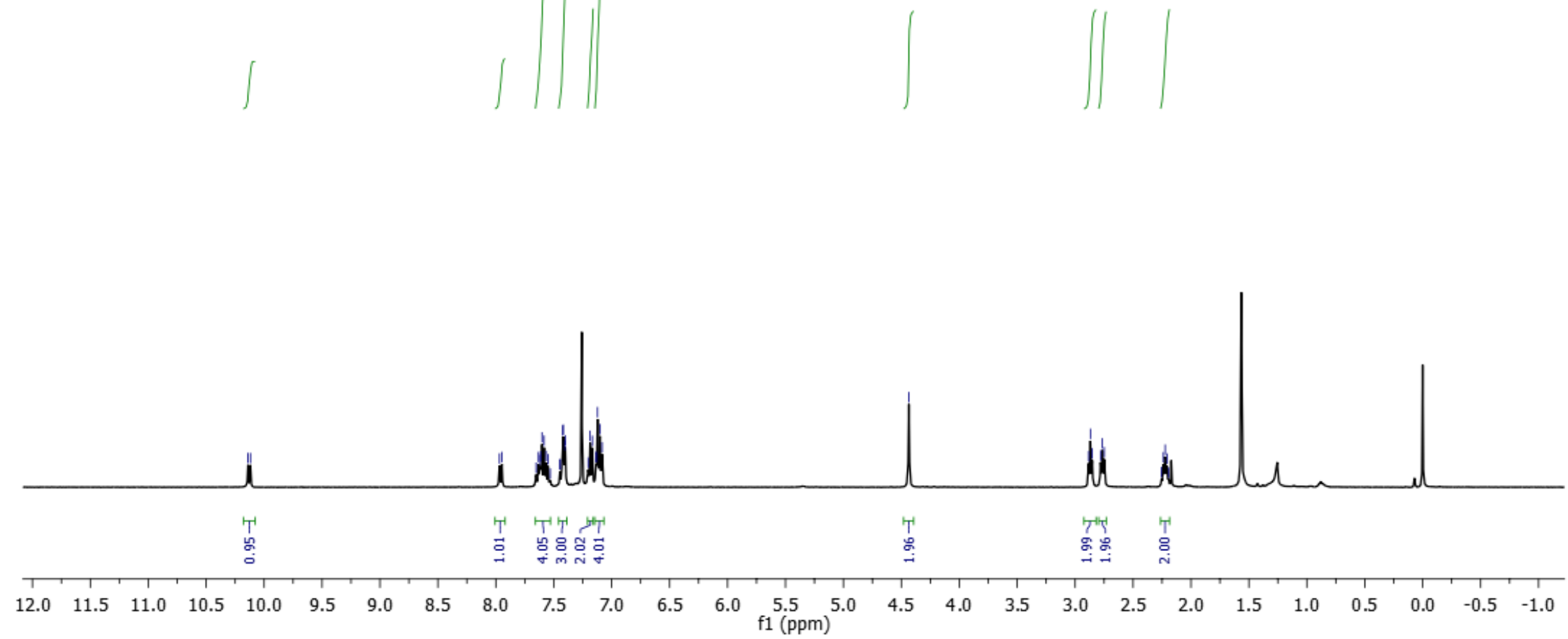


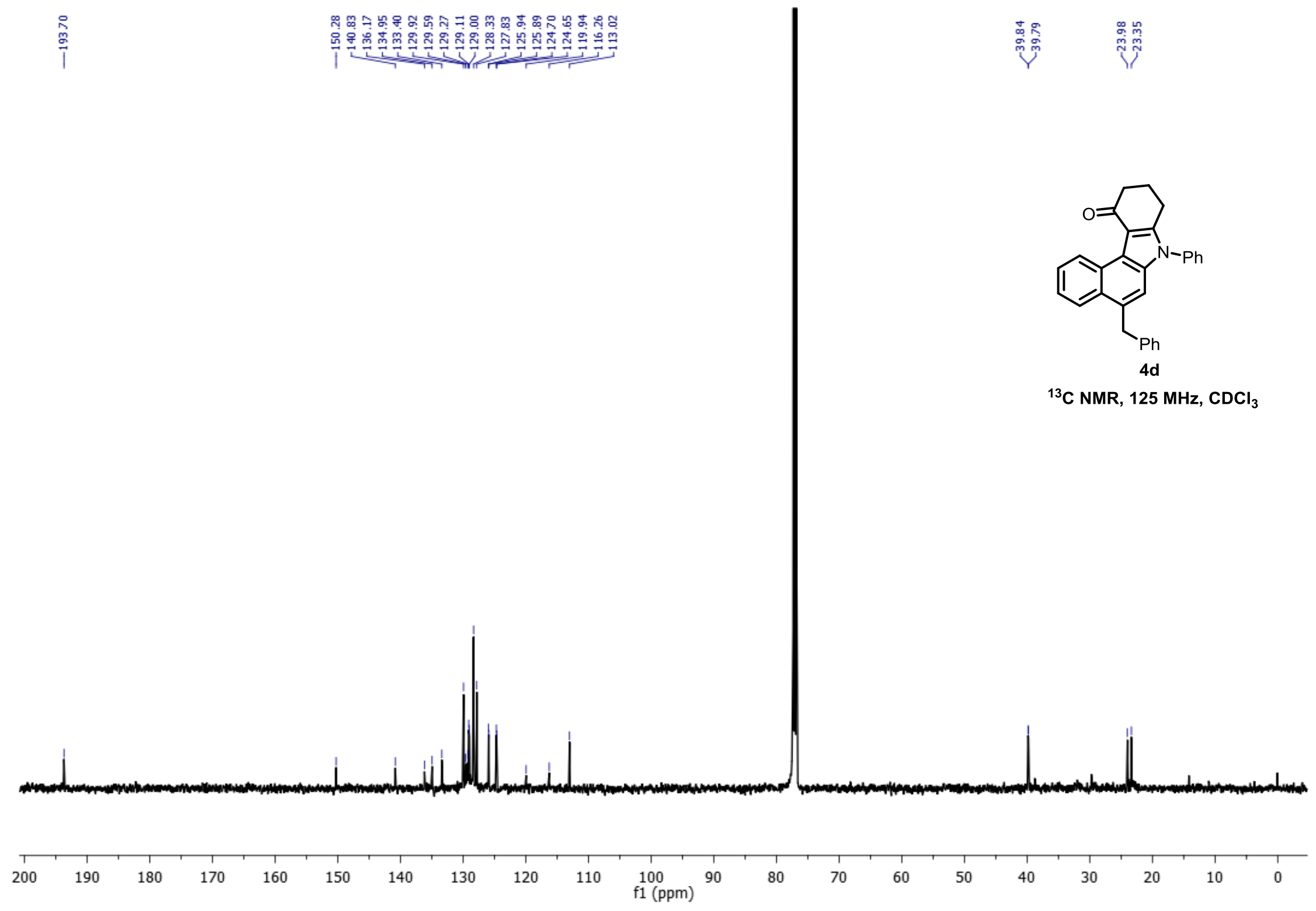




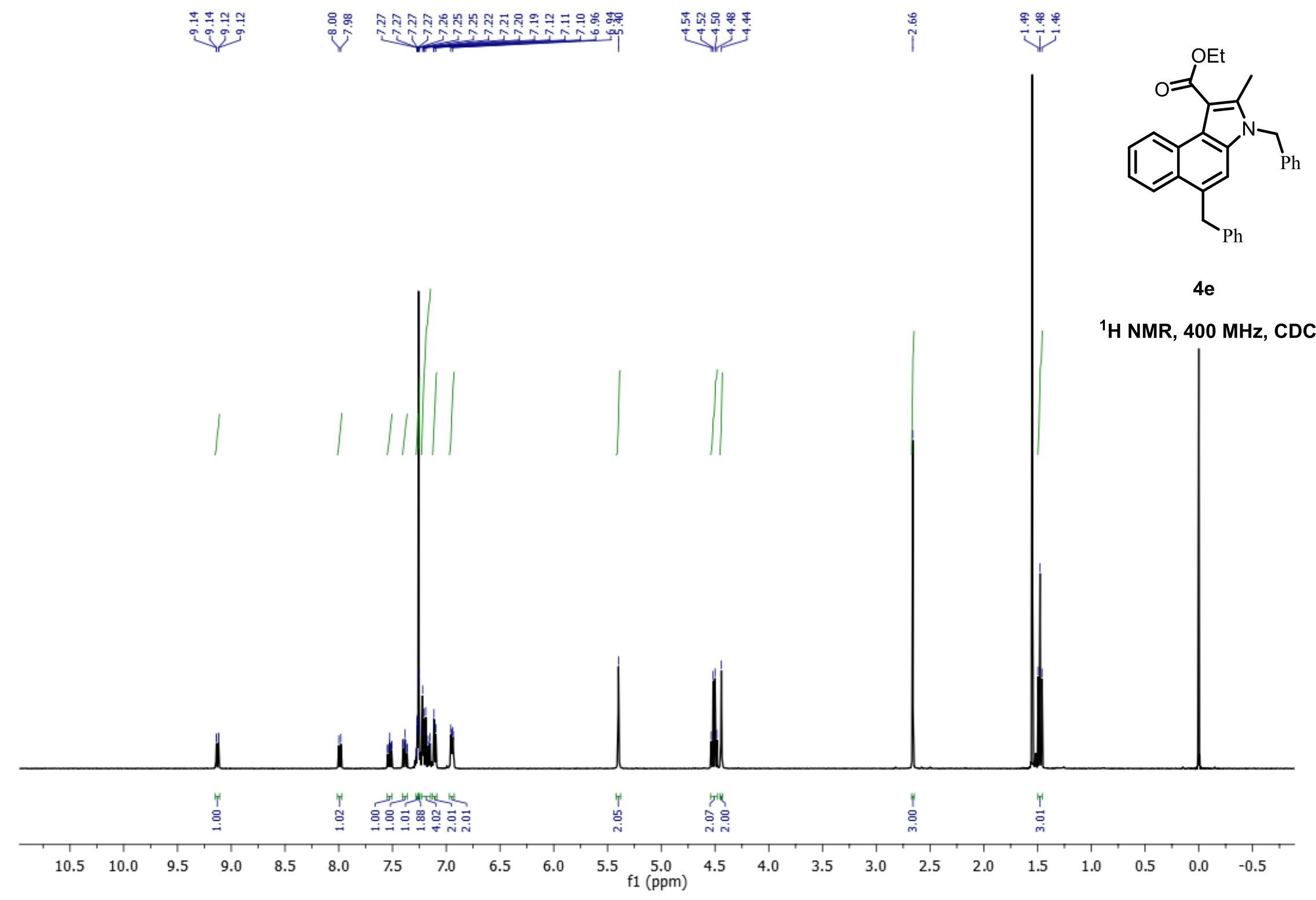




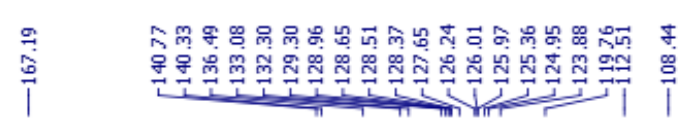

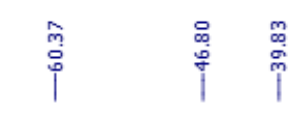

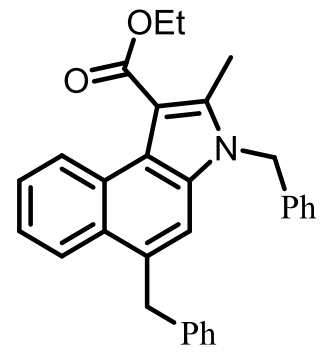

$4 e$

${ }^{13} \mathrm{C}$ NMR, $125 \mathrm{MHz}, \mathrm{CDCl}_{3}$

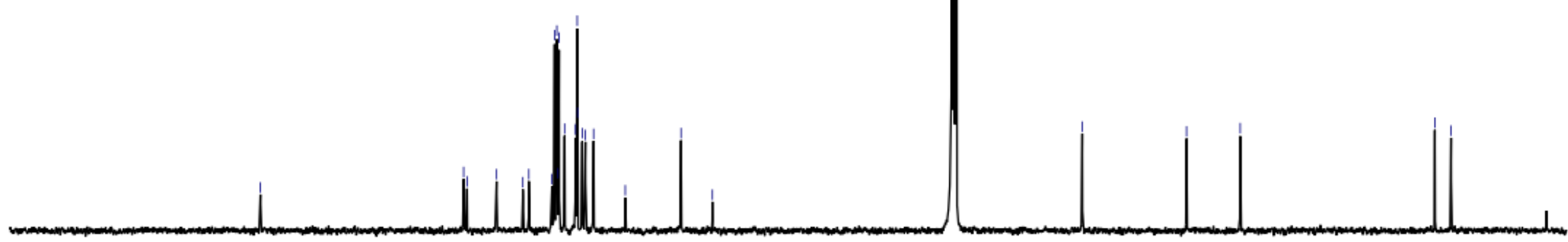

190
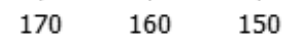

140

130

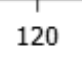

110

100

f1 (ppm) 


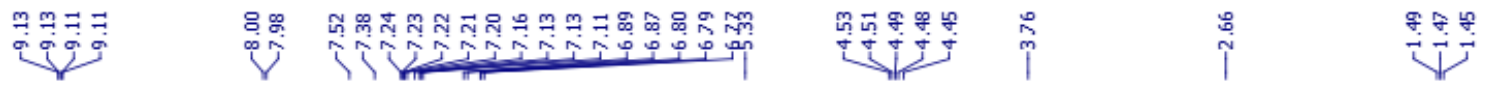

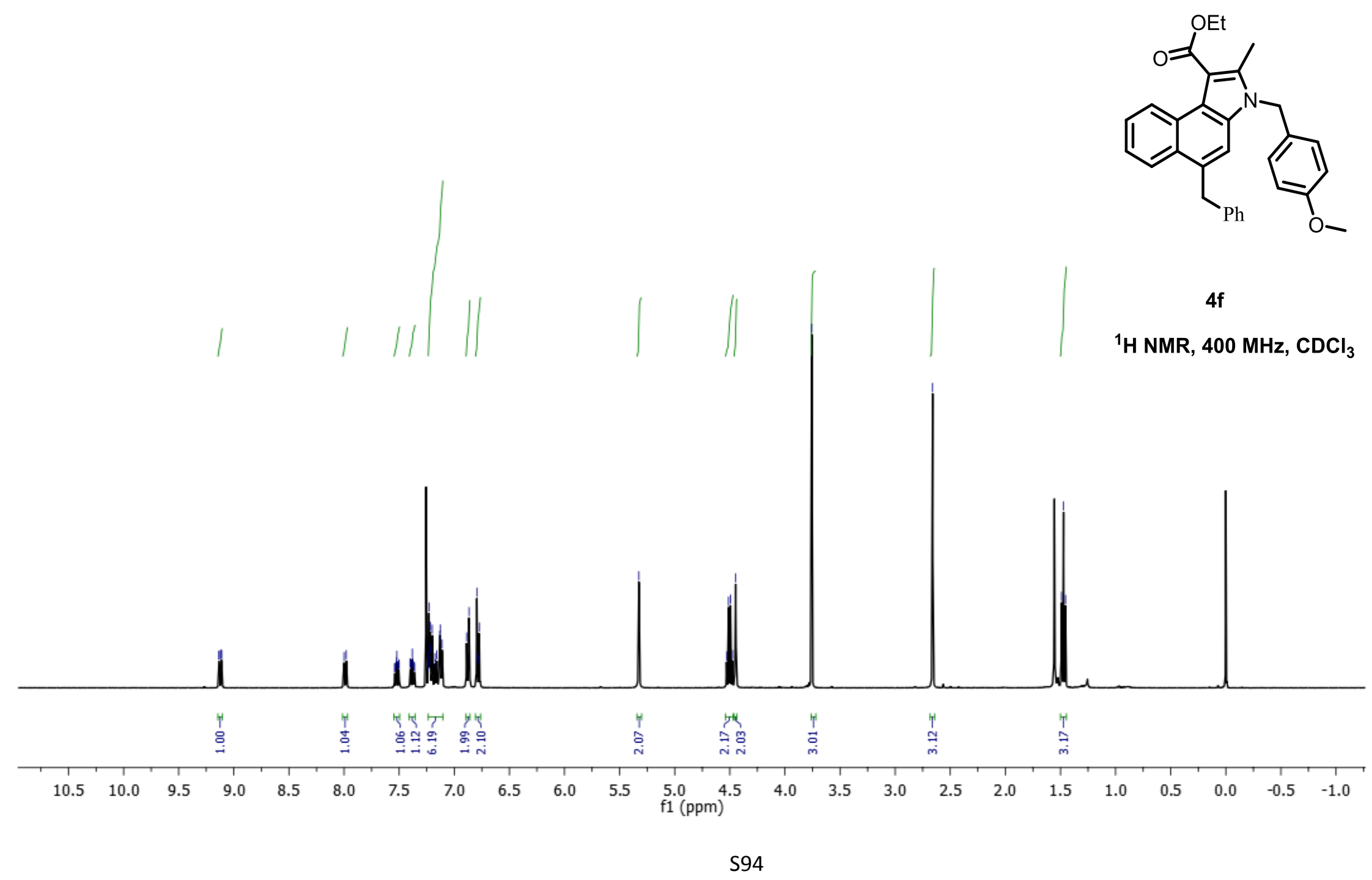




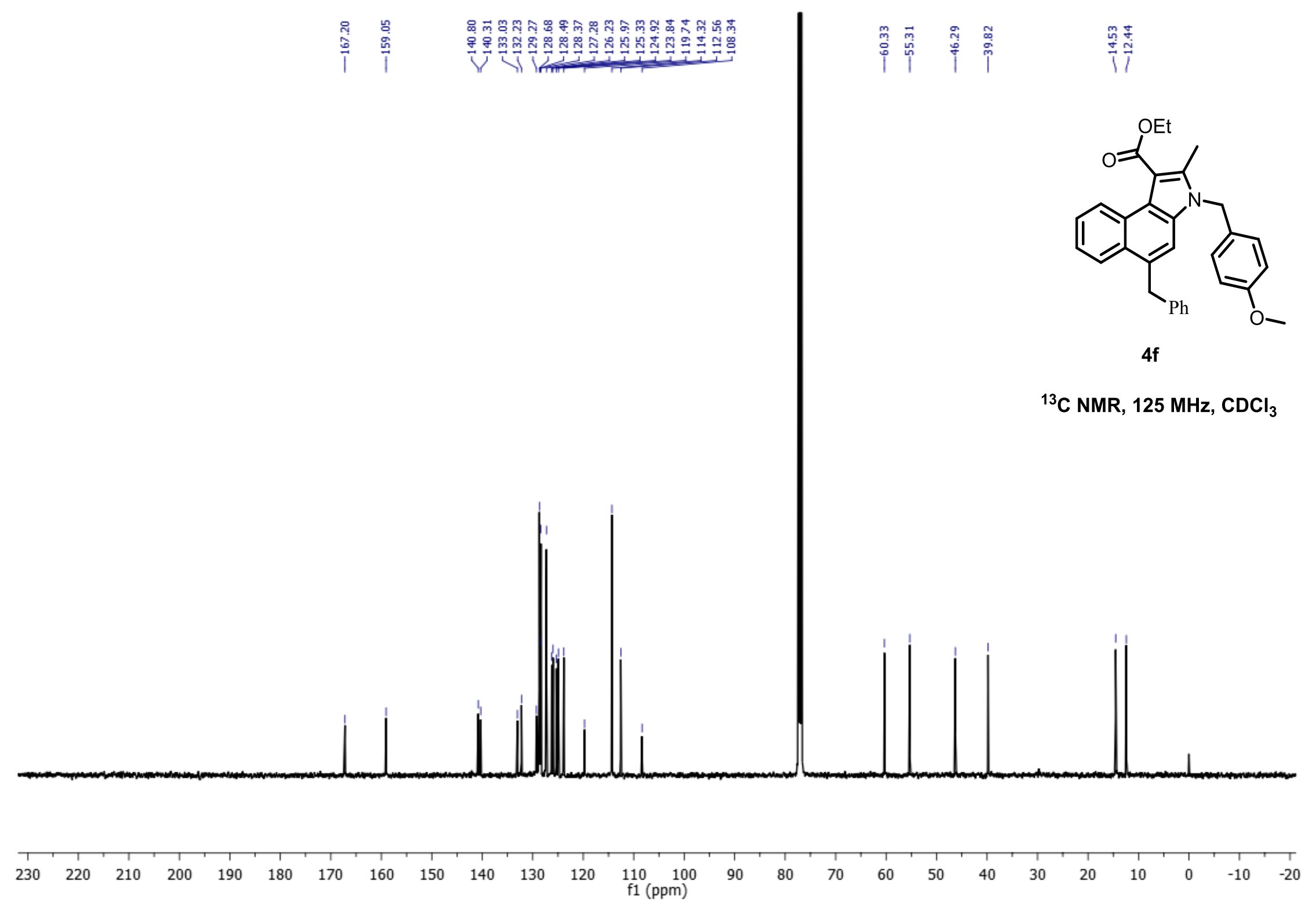




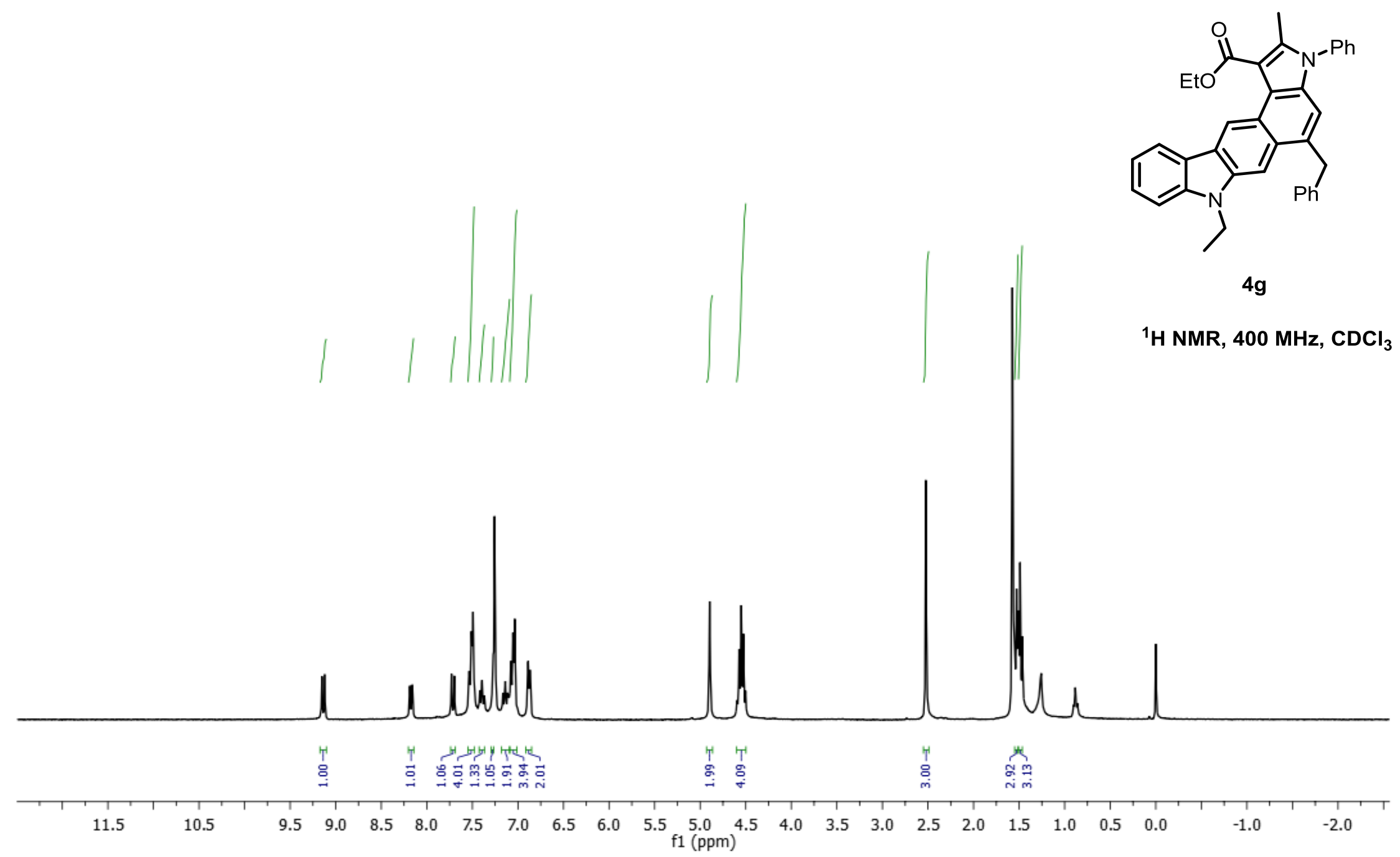



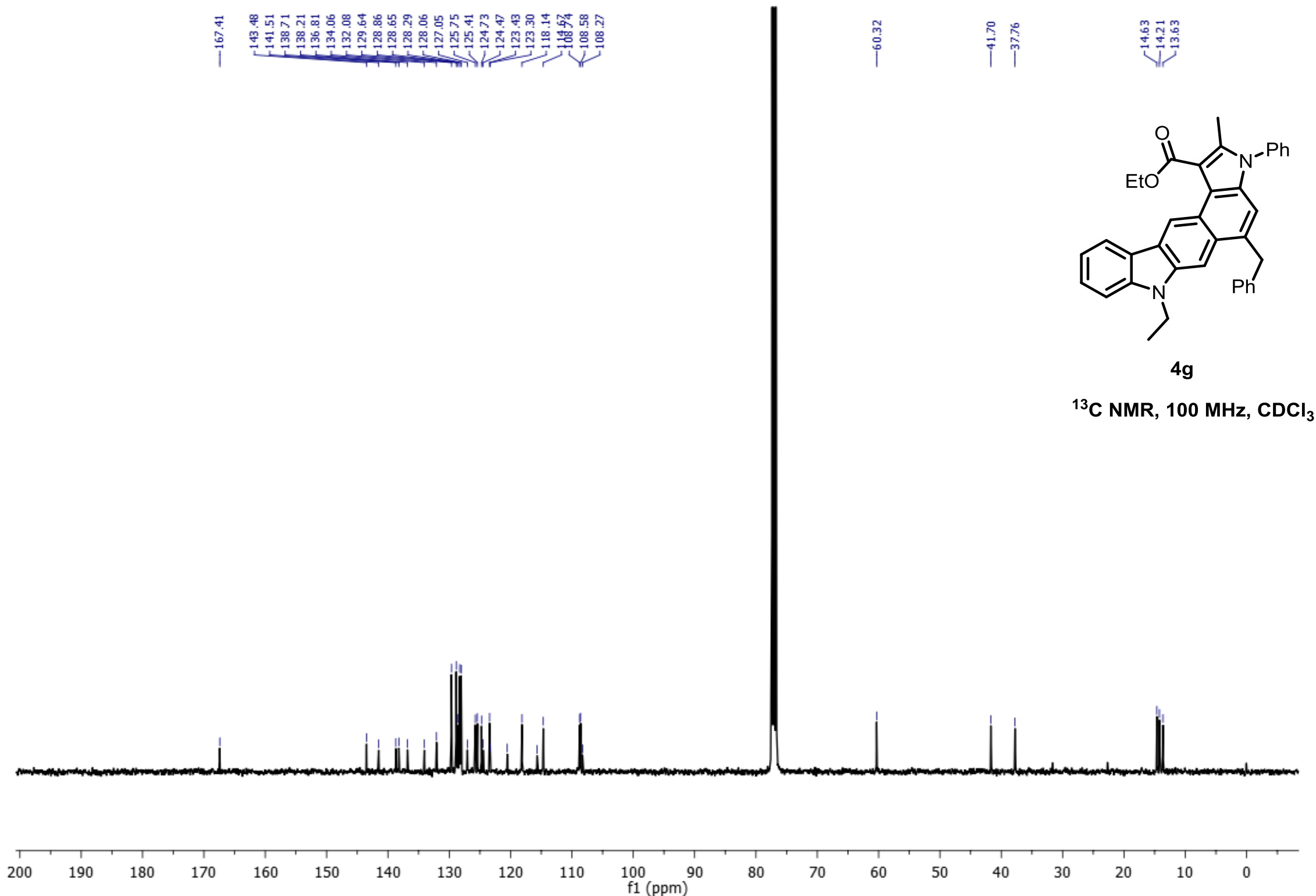

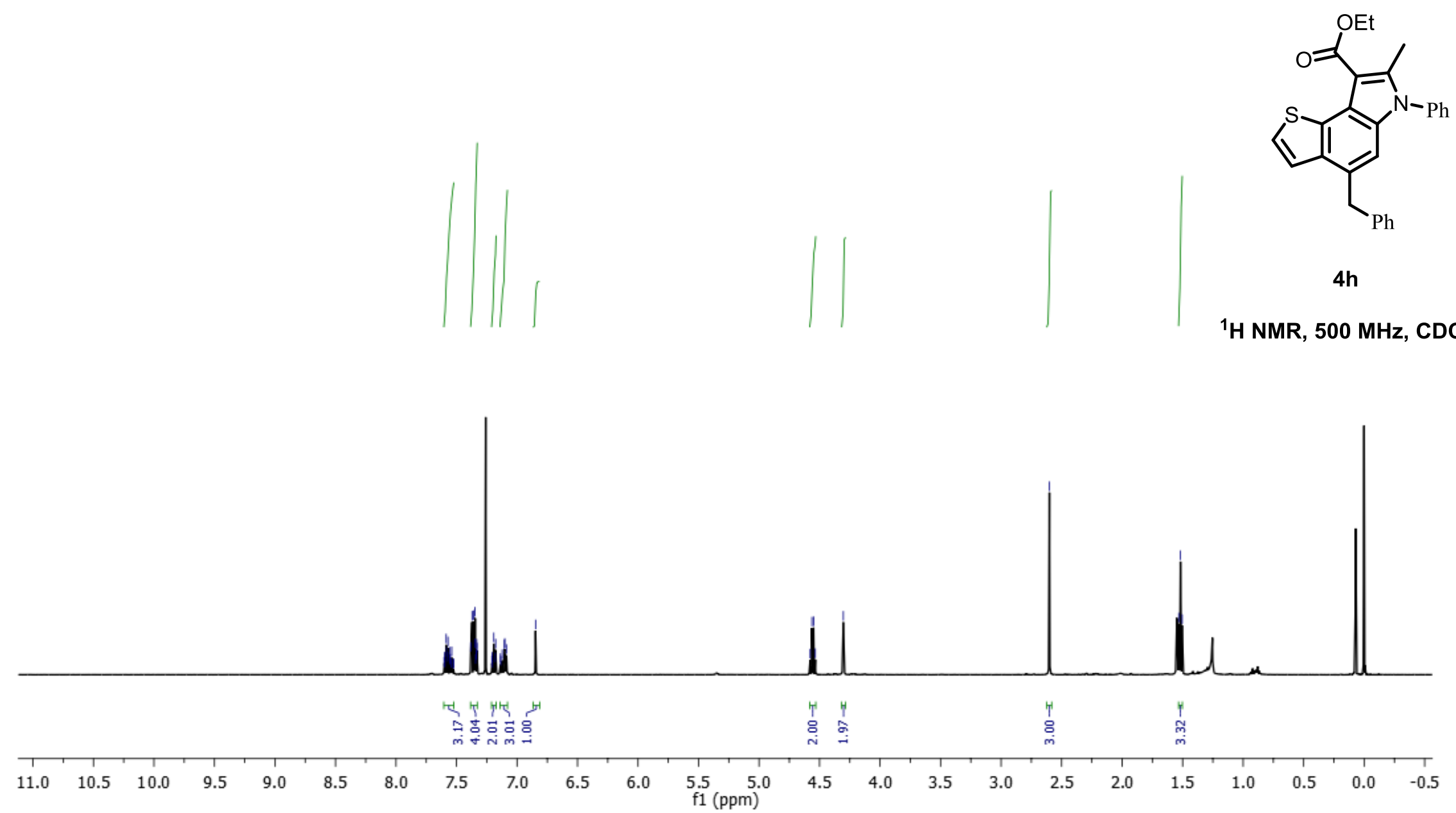


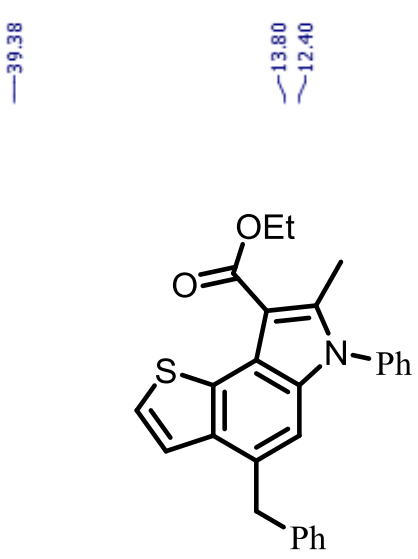

4h

${ }^{13} \mathrm{C} \mathrm{NMR}, 100 \mathrm{MHz} \mathrm{CDCl}_{3}$

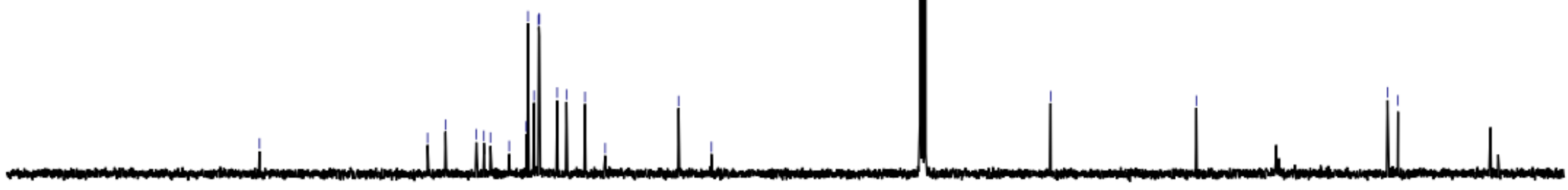

190

80

$170 \quad 160$

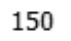

$140 \quad 130$

120

$\begin{array}{cc}100 & 90 \\ \mathrm{f} 1(\mathrm{ppm})\end{array}$ 


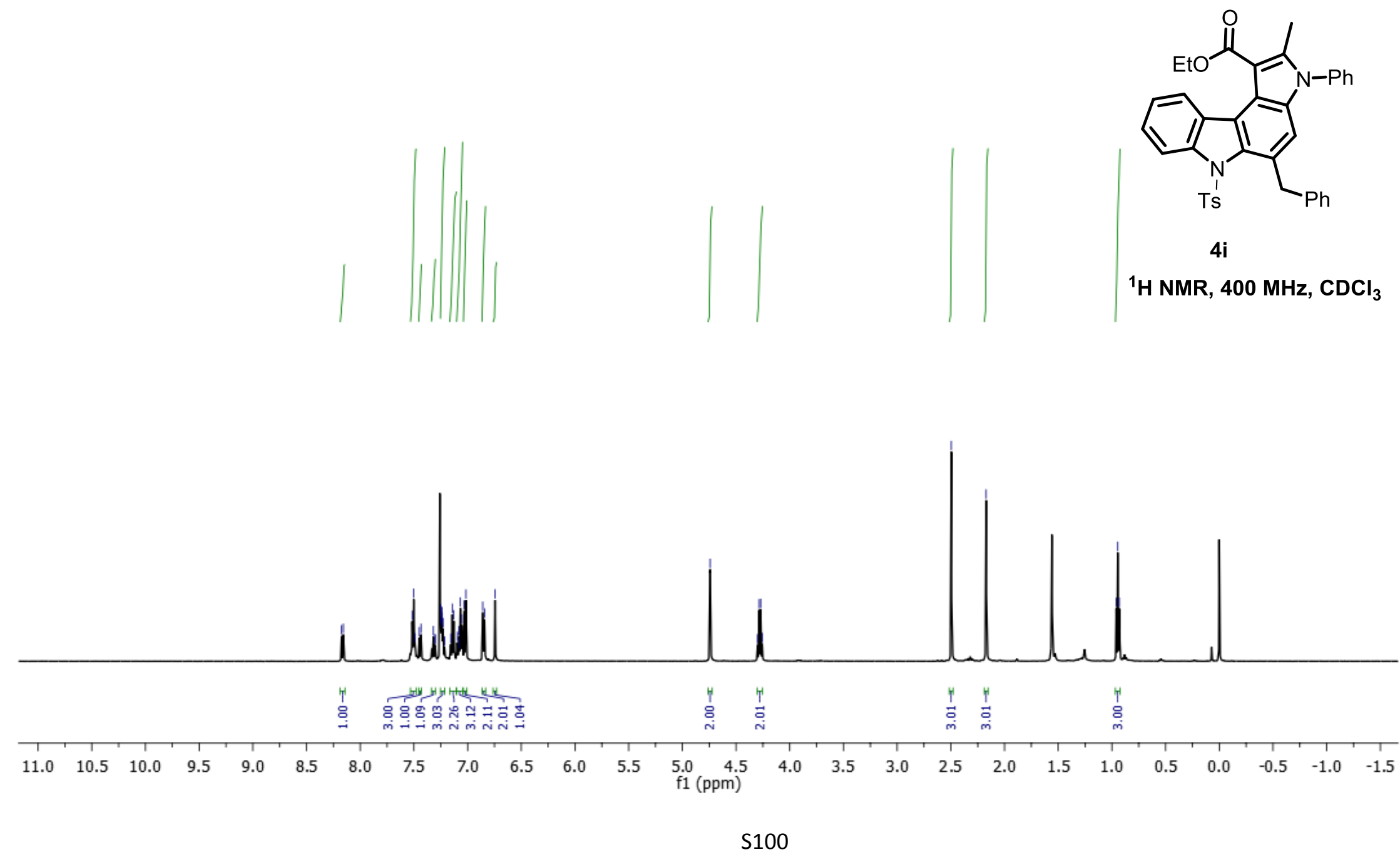




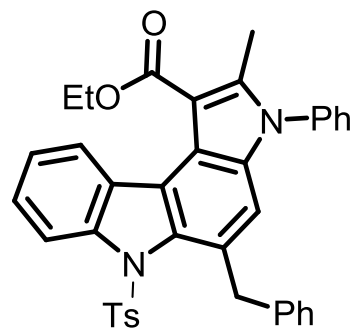

$4 i$

${ }^{13} \mathrm{C}$ NMR, $100 \mathrm{MHz}, \mathrm{CDCl}_{3}$

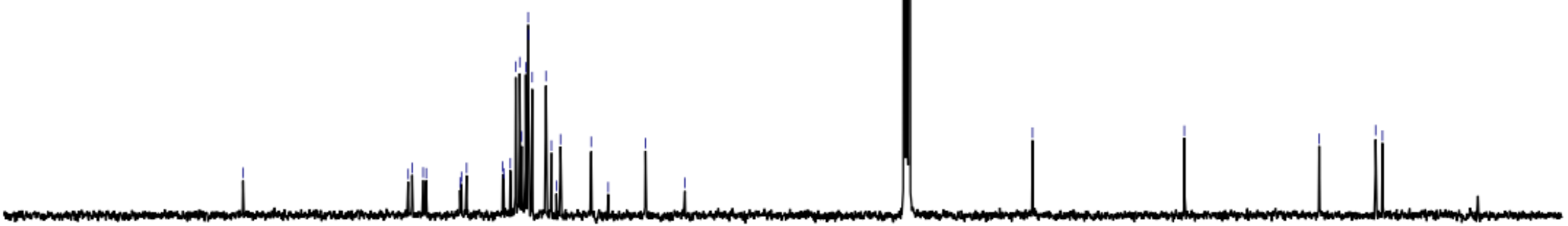




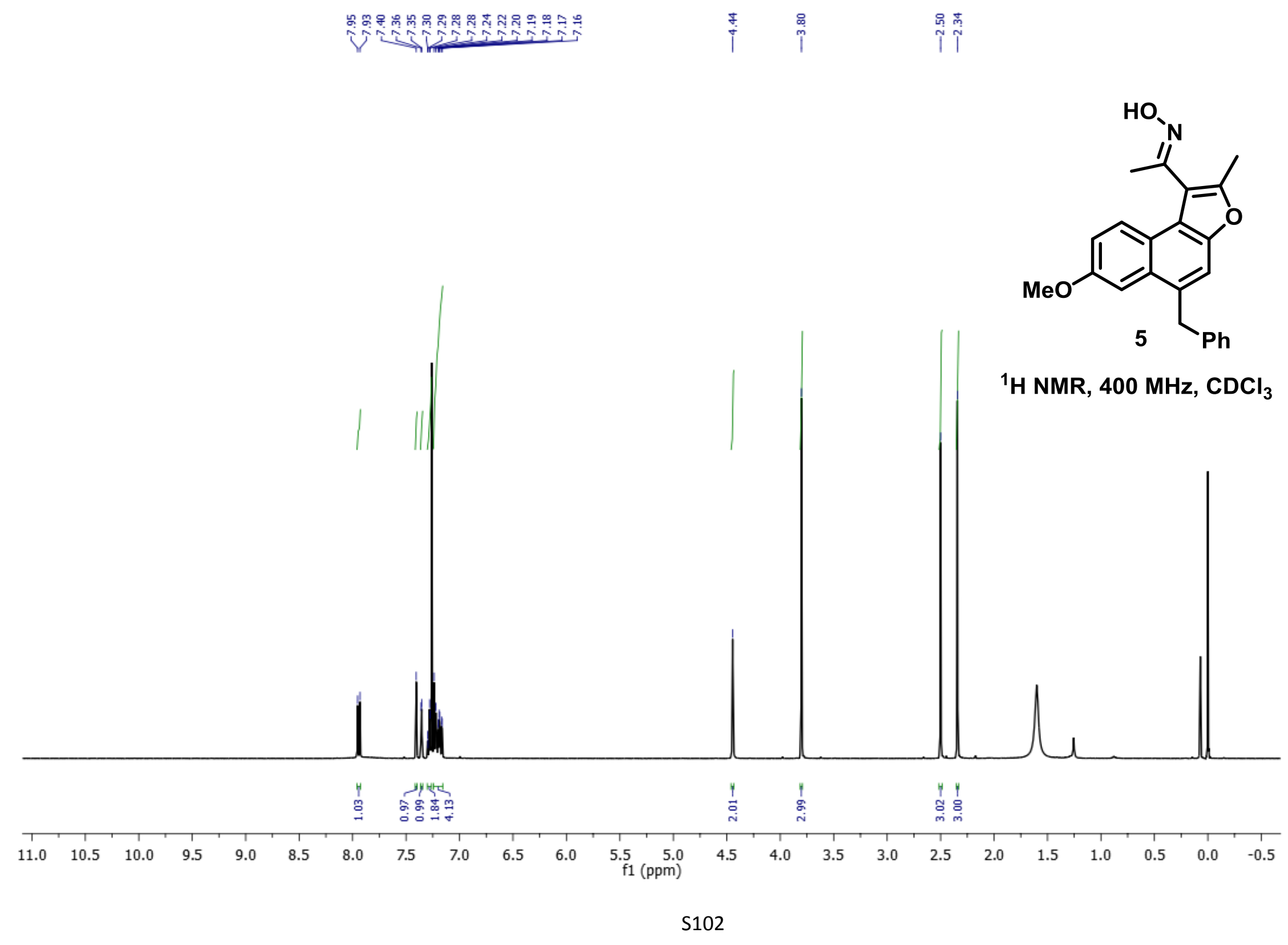




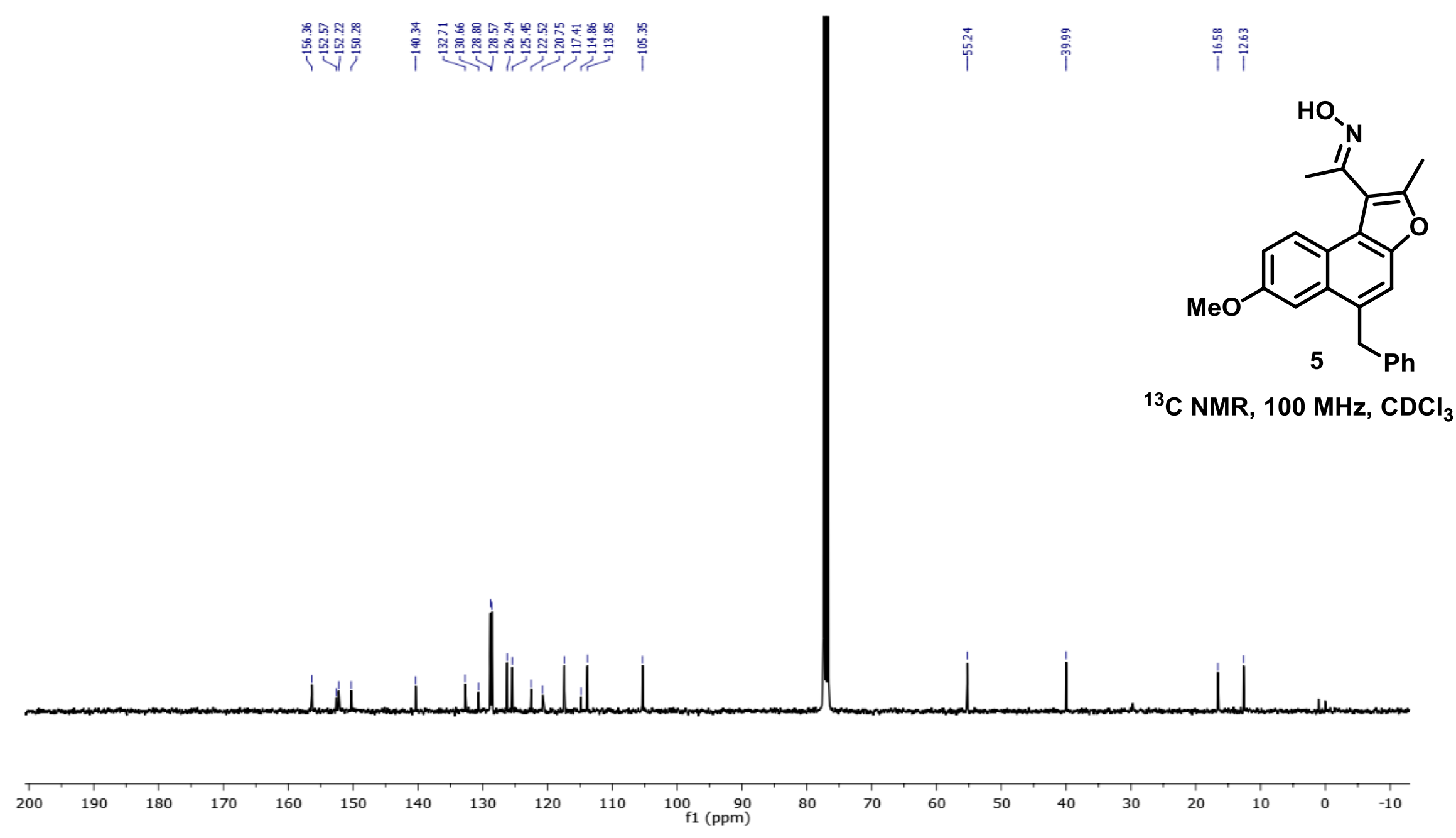

